\title{
Brønsted Acid Organocatalyzed Three-Component Hydroamidation Reactions of Vinyl Ethers
}

\author{
Ivor Smajlagic, Brenden Carlson and Travis Dudding* \\ Department of Chemistry, Brock University, 1812 Sir Isaac Brock Way, St. Catharines, ON L2S \\ 3A1, Canada \\ *Email: tdudding@brocku.ca
}

\section{SUPPORTING INFORMATION}

\section{Table of Contents:}

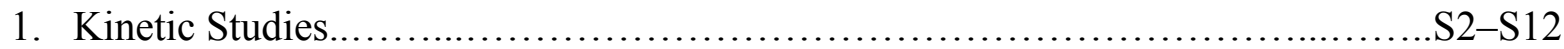

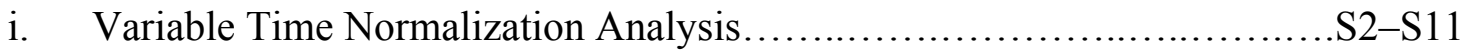

ii. Kinetic Solvent Isotope Effect Studies....................................... 12

2. Experimental Evidence Supporting CSA-Mediated Catalysis.......................S12-S13

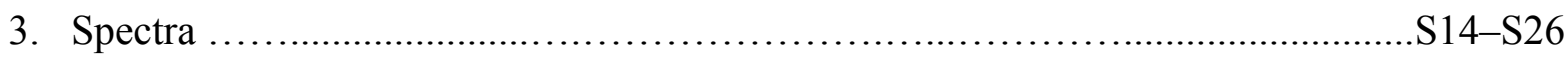

4. Cartesian Coordinates and Energies of Calculated Structures...........................S27-S52

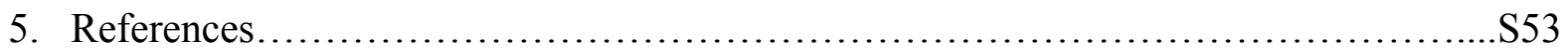




\section{Kinetic studies}

\section{i. $\quad$ Variable Time Normalization Analysis}

Initial efforts to help delineate the hydroamidation mechanism involved performing a series of comparative VTNA experiments. ${ }^{1}$ Exploiting this methodology helped provide key mechanistic facets of reactivity, including catalyst deactivation, the order in catalyst, as well as the order in substrates. The order in catalyst was determined abiding to the normalized time scale method, which was approximated via the trapezoid rule, and, thus, removes the kinetic effect of that component from the reaction profile. Notably, VTNA can be used to elucidate numerous reaction components irrespective of whether their concentration is constant or variable. To this end, the following equations were used to determine the order in catalyst (eq 1) and the order in substrates (eq 2). The value of " $\gamma$ " and " $\beta$ " that provides the greatest overlay of the generated curves is the order in the respective component.

$$
\begin{aligned}
& \sum[\text { cat }]^{\gamma} \Delta t=\sum_{i=1}^{n}\left(\frac{[\mathrm{cat}]_{i}+[\mathrm{cat}]_{i-1}}{2}\right)^{\gamma}\left(t_{i}-t_{i-1}\right) \\
& \sum[\text { component }]^{\beta} \Delta t=\sum_{i=1}^{n}\left(\frac{[\text { component }]_{i}+[\text { component }]_{i-1}}{2}\right)^{\beta}\left(t_{i}-t_{i-1}\right)
\end{aligned}
$$


Scheme S1. Investigating the order in substrates via VTNA using ethyl vinyl ether.

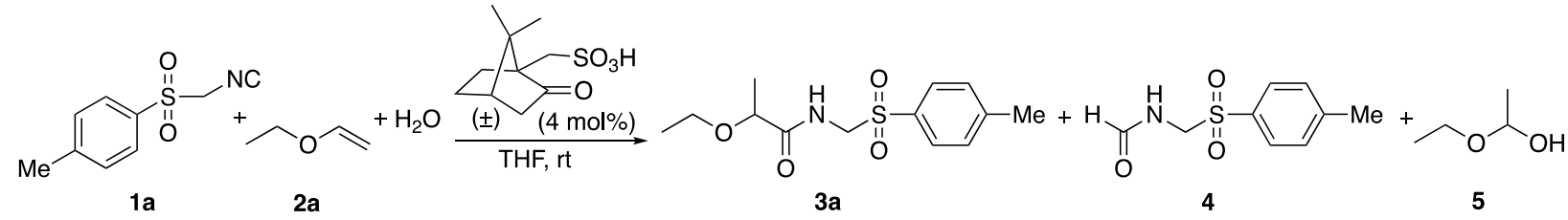

Table S1. Experimental conditions for determining the order in reagents using ethyl vinyl ether.

$\begin{array}{cccccccc} & \begin{array}{c}\text { Expt 1 } \\ \text { (std. cond.) }\end{array} & \text { Expt 2 } & \text { Expt 3 } & \text { Expt 4 } & \text { Expt 5 } & \text { Expt 6 } & \text { Expt 7 } \\ {[\mathbf{1 a}]_{\mathrm{o}}(\mathrm{M})} & 2.4 & 1.8 & 1.4 & 2.4 & 2.4 & 2.4 & 2.4 \\ {[\mathbf{2 a}]_{\mathrm{o}}(\mathrm{M})} & 5.9 & 5.9 & 5.9 & 4.1 & 3.0 & 5.9 & 5.9 \\ {\left[\mathrm{H}_{2} \mathrm{O}\right]_{\mathrm{o}}(\mathrm{M})} & 3.9 & 3.9 & 3.9 & 3.9 & 3.9 & 3.1 & 2.5 \\ {[\mathrm{CSA}]_{\mathrm{o}}(\mathrm{M})} & 0.095 & 0.095 & 0.095 & 0.095 & 0.095 & 0.095 & 0.095 \\ {[\text { reduction] (M) }} & - & 0.6 & 1.0 & 1.8 & 2.9 & 0.8 & 1.4\end{array}$

VTNA Data Illustrating the "Best” Overlay of the Curves using Arbitrary Exponents

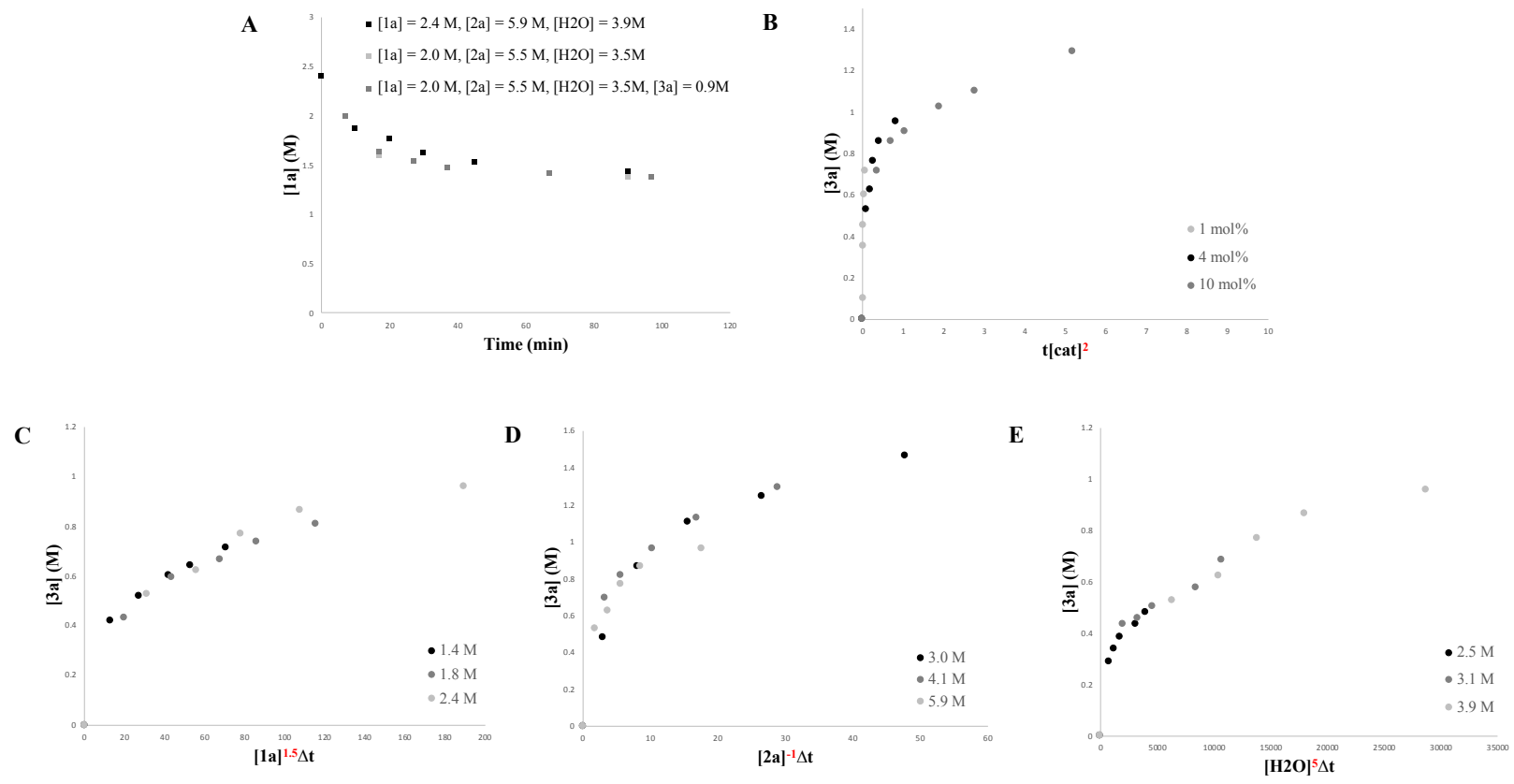

Figure S1. VTNA experimental data with respect to isocyanide consumption for product formation using ethyl vinyl ether as vinyl ether component. (A) Plot depicting mild catalyst deactivation. (B) Normalized time scale plot displaying second-order in catalyst behavior. (C-E) Plots revealing concentration dependences of substrates 1a, 2a, and $\mathrm{H}_{2} \mathrm{O}$ indicating positive 1.5order, negative first-order, and positive fifth-order, respectively. 

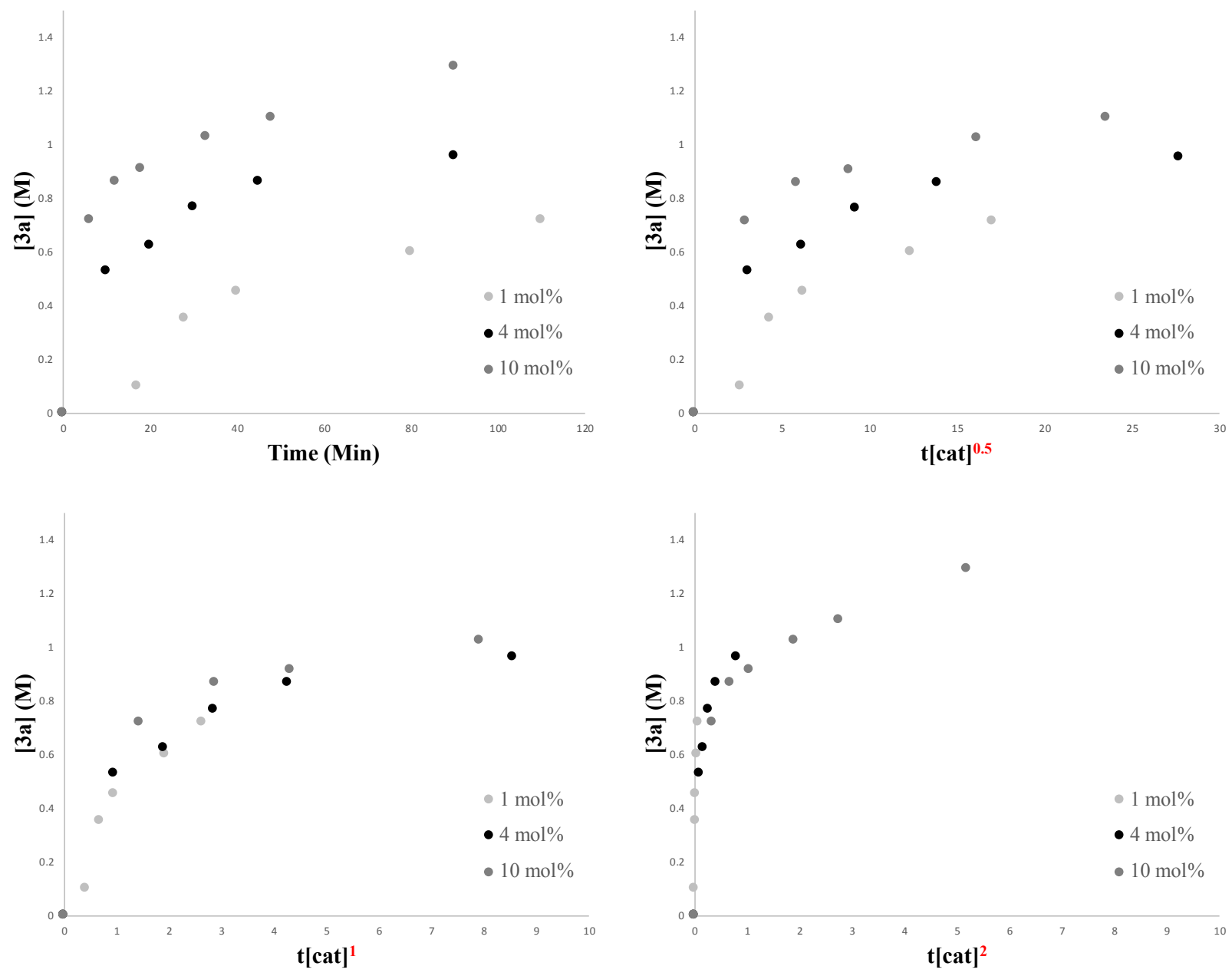

Figure S2. VTNA data indicating optimal overlay using exponent "2" for determining the order in catalyst. 

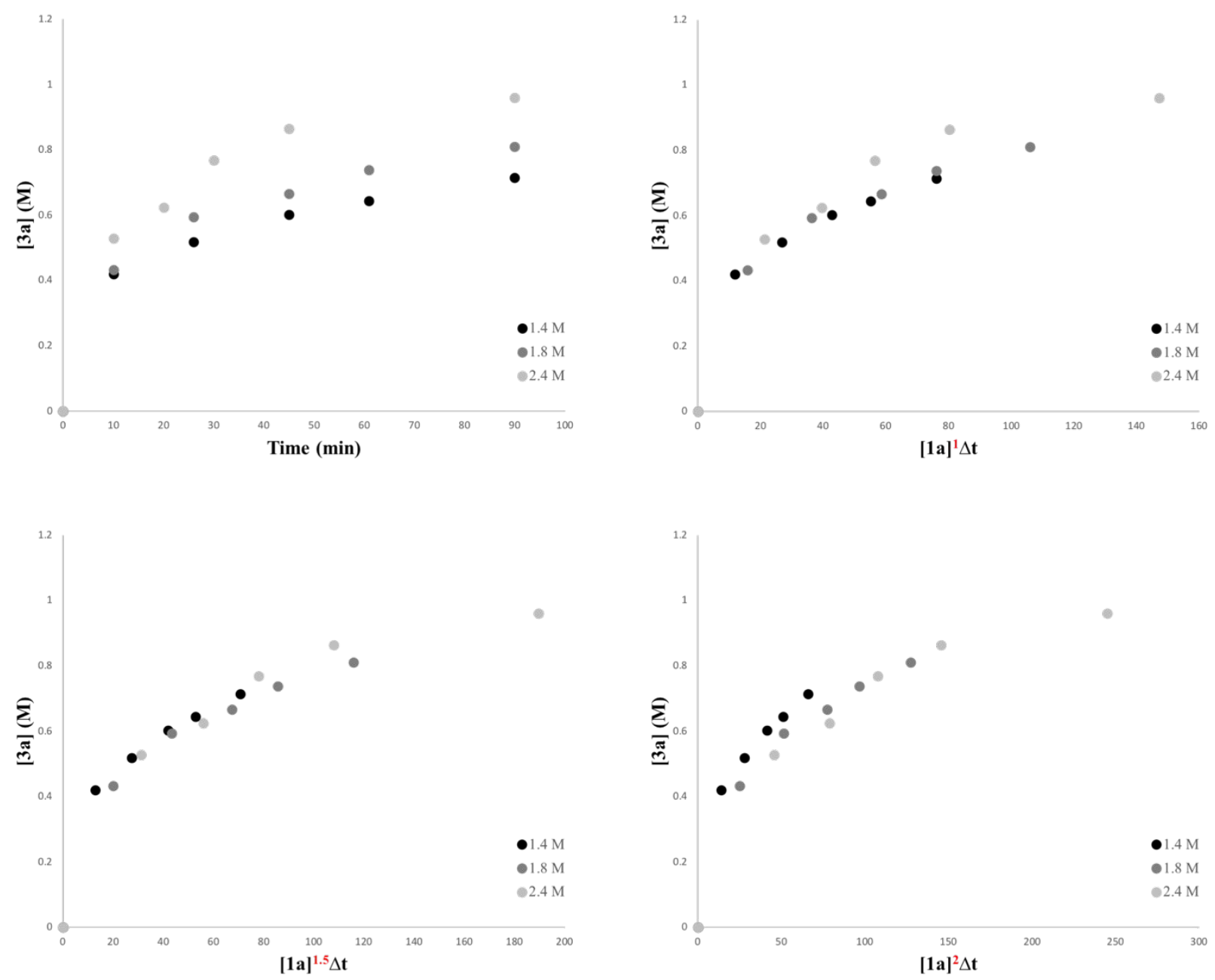

Figure S3. VTNA data indicating optimal overlay using exponent " 1.5 " for determining the order in isocyanide 1a. 

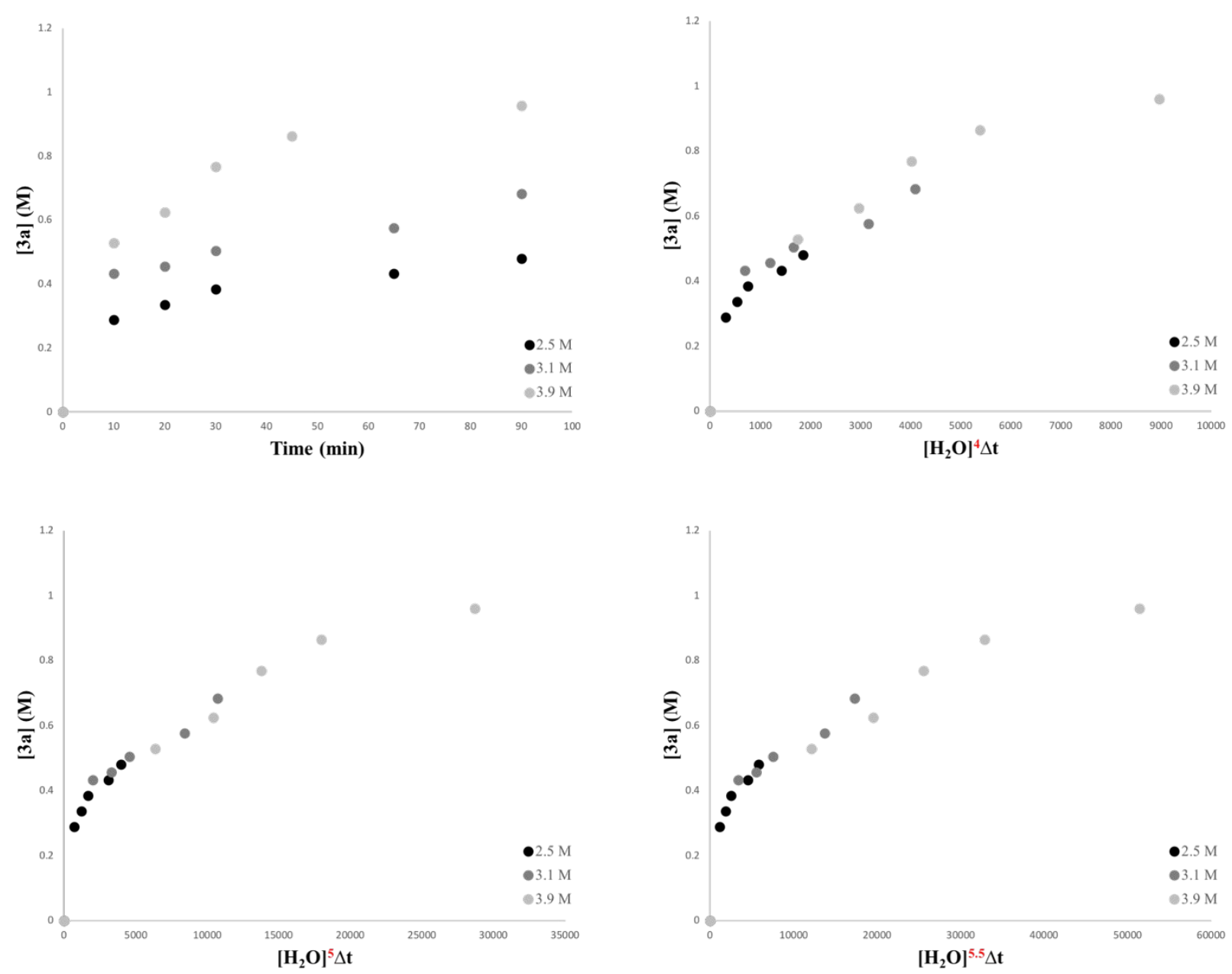

Figure S4. VTNA data indicating optimal overlay using exponent "5" for determining the order in $\mathrm{H}_{2} \mathrm{O}$.

The above trends were realized in the presence of more "clean" reaction conditions with minimal side product formation, and, thus, surmounting the possibility of abating the product formation pathway. The next set of VTNA experiments sought to probe the mechanism using DHP (2b) as the vinyl ether component, which led to a higher population of side products. This comparative kinetic analysis would reveal if a change in the mechanism transpires. The corresponding VTNA studies employing DHP as substrate follow below. 
Scheme S2. Investigating the order in substrates via VTNA using DHP.

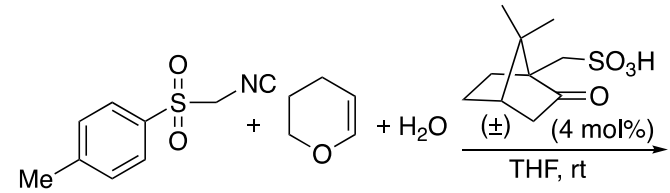

$1 a$

$2 b$

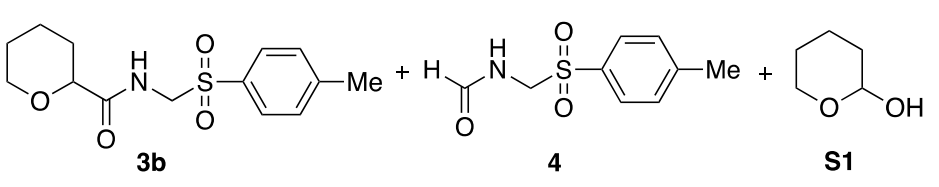

Table S2. Experimental conditions for determining the order in reagents using DHP.

\begin{tabular}{cccccccc} 
& $\begin{array}{c}\text { Expt 1 } \\
\text { (std. cond.) }\end{array}$ & Expt 2 & Expt 3 & Expt 4 & Expt 5 & Expt 6 & Expt 7 \\
\hline $\mathbf{1 a}]_{\mathrm{o}}(\mathrm{M})$ & 2.4 & 1.8 & 1.4 & 2.4 & 2.4 & 2.4 & 2.4 \\
{$[\mathbf{2 b}]_{\mathrm{o}}(\mathrm{M})$} & 5.9 & 5.9 & 5.9 & 4.1 & 3.0 & 5.9 & 5.9 \\
{$\left[\mathrm{H}_{2} \mathrm{O}\right]_{\mathrm{o}}(\mathrm{M})$} & 3.9 & 3.9 & 3.9 & 3.9 & 3.9 & 3.1 & 2.5 \\
{$[\mathrm{CSA}]_{\mathrm{o}}(\mathrm{M})$} & 0.095 & 0.095 & 0.095 & 0.095 & 0.095 & 0.095 & 0.095 \\
{$[$ reduction] $(\mathrm{M})$} & - & 0.6 & 1.0 & 1.8 & 2.9 & 0.8 & 1.4
\end{tabular}

VTNA Data Illustrating the "Best" Overlay of the Curves using Arbitrary Exponents

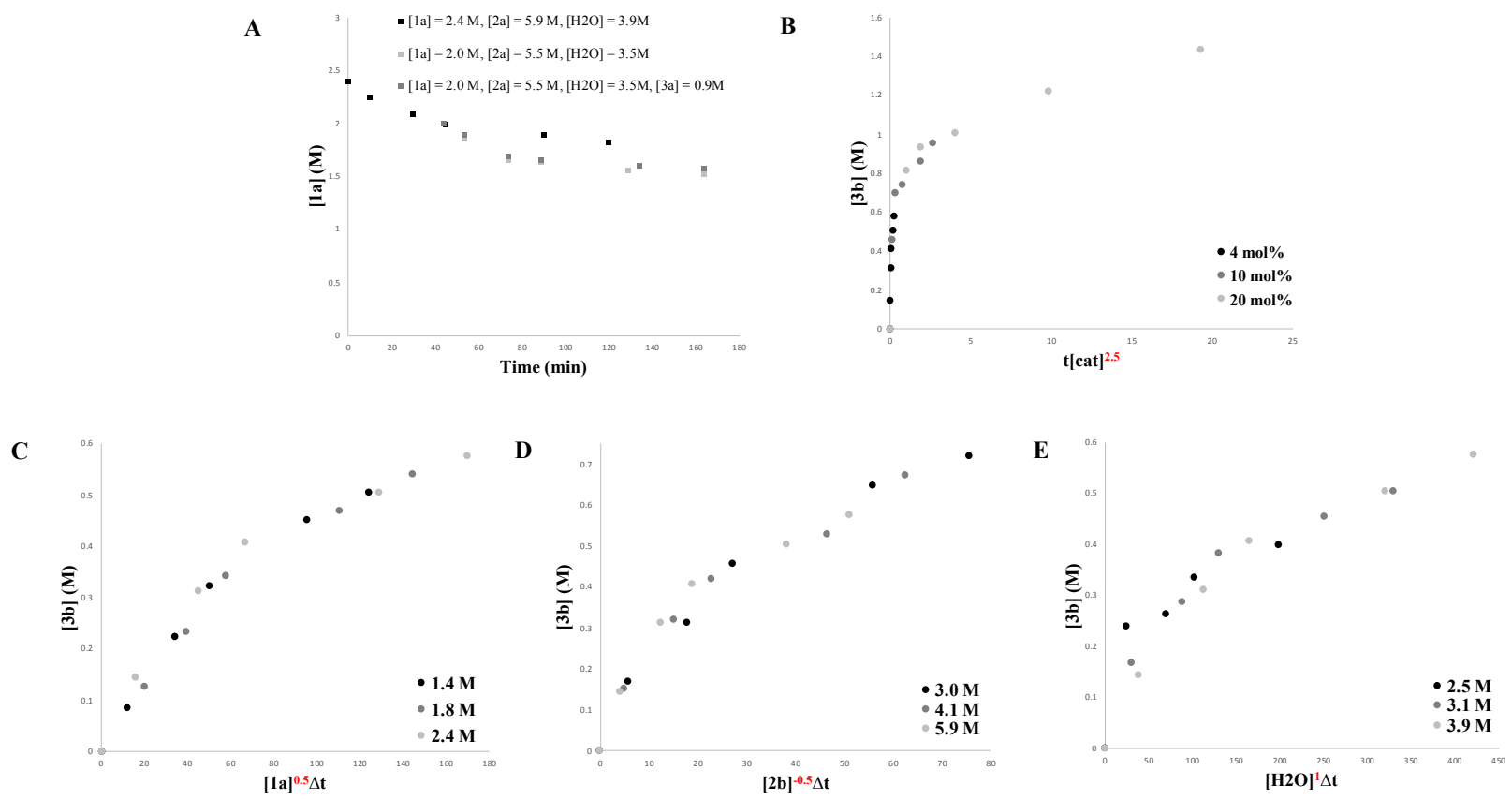

Figure S5. VTNA experimental data with respect to isocyanide consumption for product formation using DHP as vinyl ether component. (A) Plot depicting moderate catalyst deactivation. 
(B) Normalized time scale plot displaying 2.5-order in catalyst. (C-E) Plots revealing concentration dependences of substrates $\mathbf{1 a}, \mathbf{2 b}$, and $\mathrm{H}_{2} \mathrm{O}$ indicating positive 0.5 -order, negative 0.5 -order, and positive first-order, respectively.
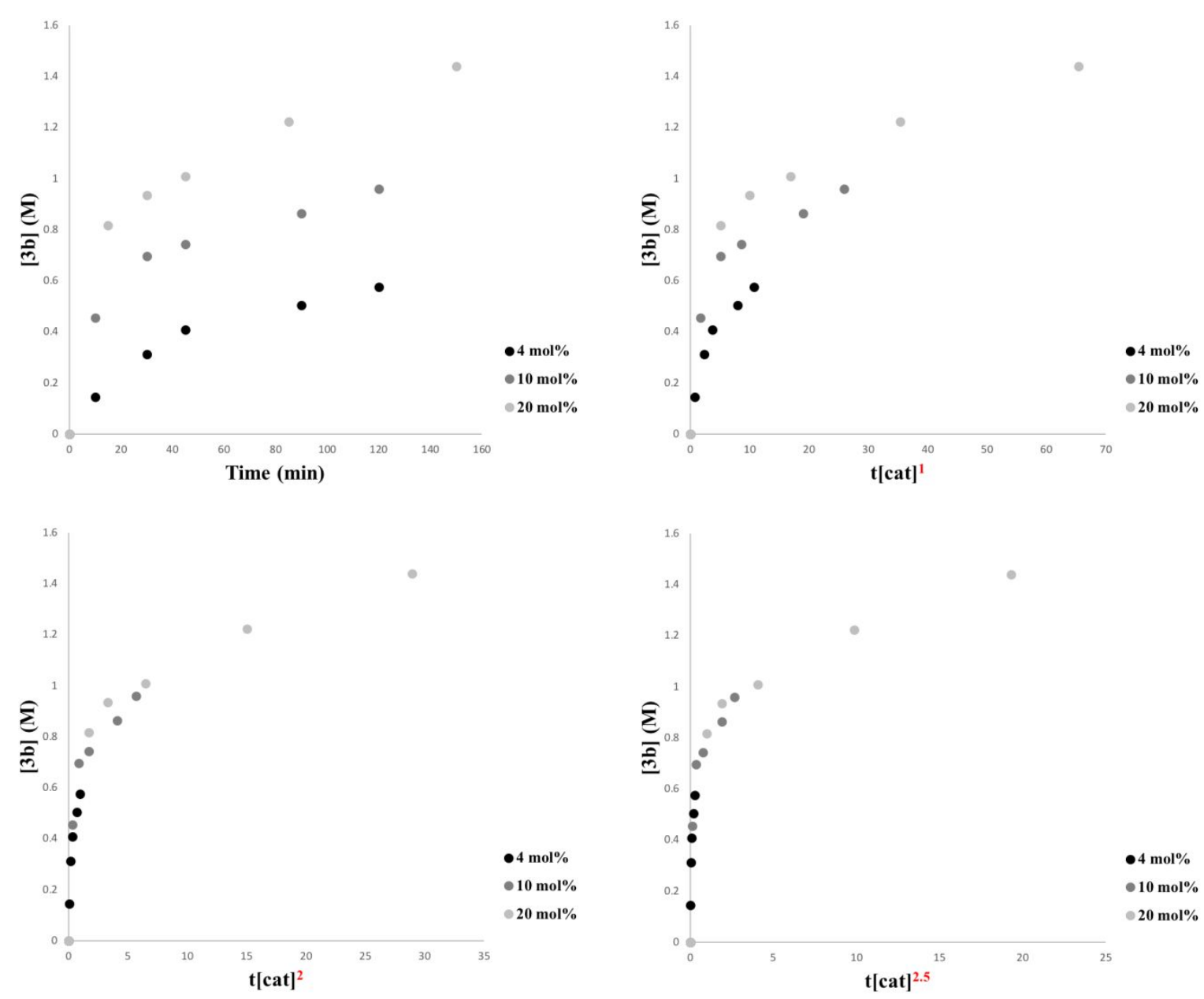

Figure S6. VTNA data indicating optimal overlay using exponent " 2.5 " for determining the order in catalyst. 

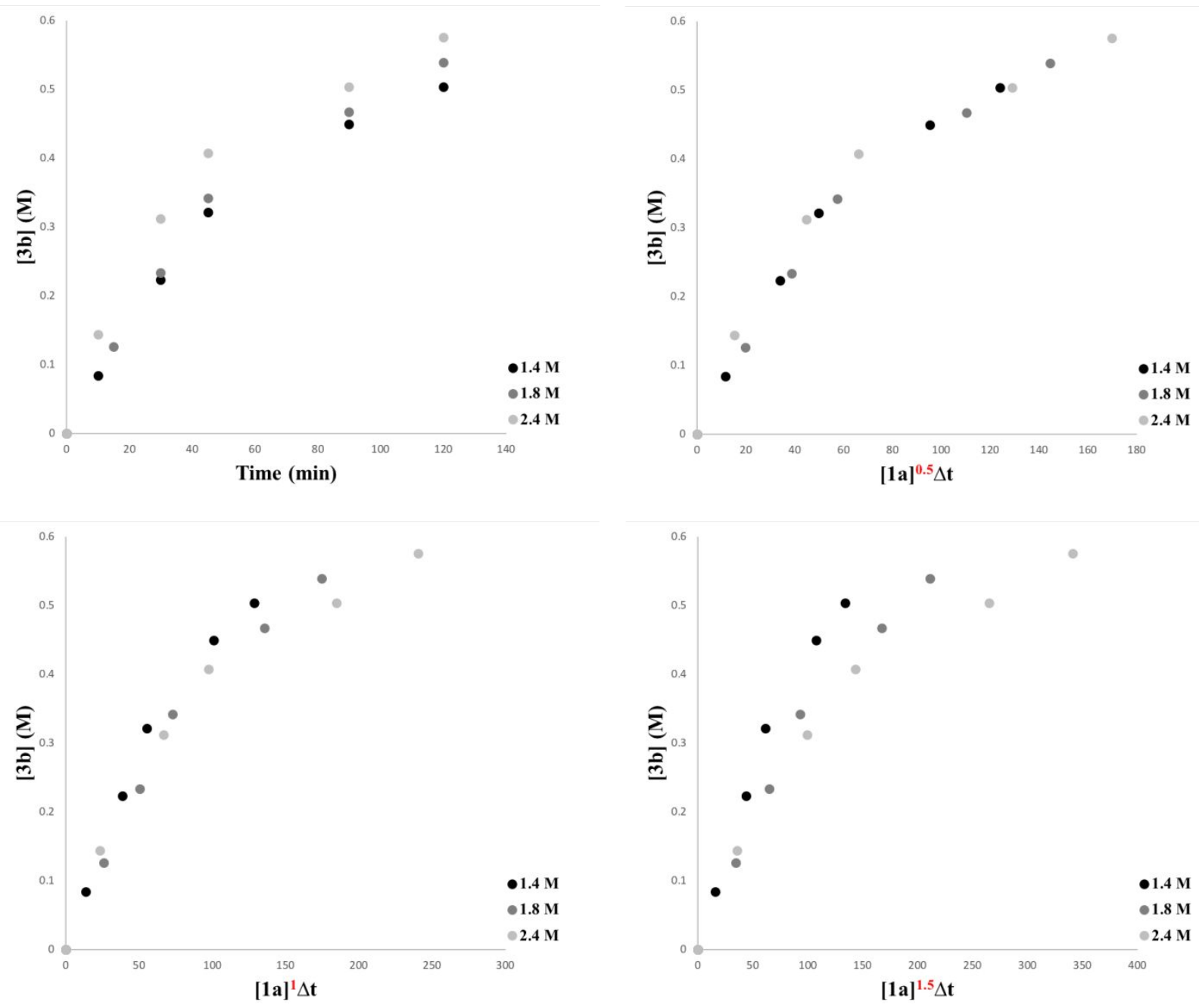

Figure S7. VTNA data indicating optimal overlay using exponent " 0.5 " for determining the order in isocyanide $\mathbf{1 a}$. 

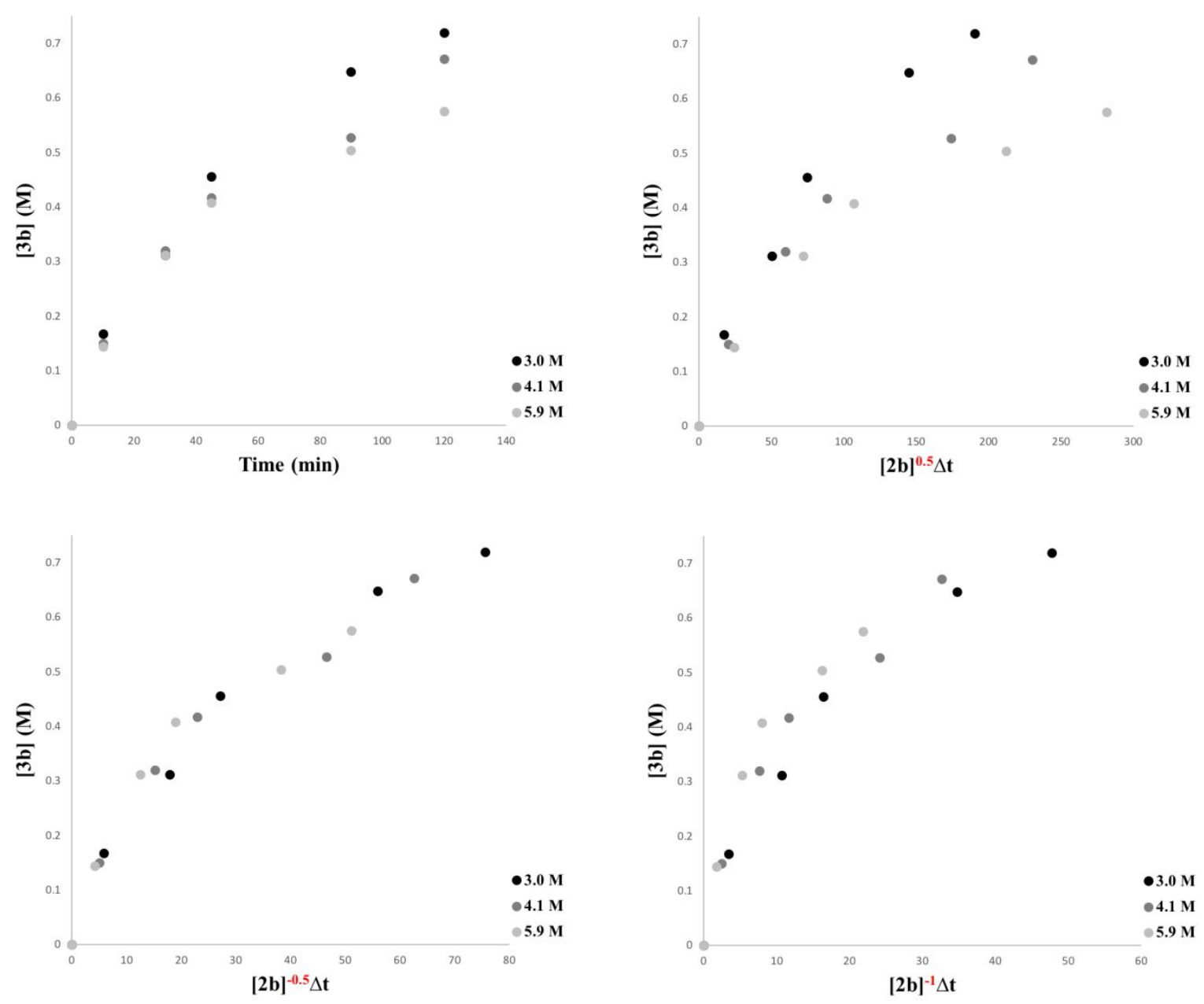

Figure S8. VTNA data indicating optimal overlay using exponent " -0.5 " for determining the order in DHP (2b). 

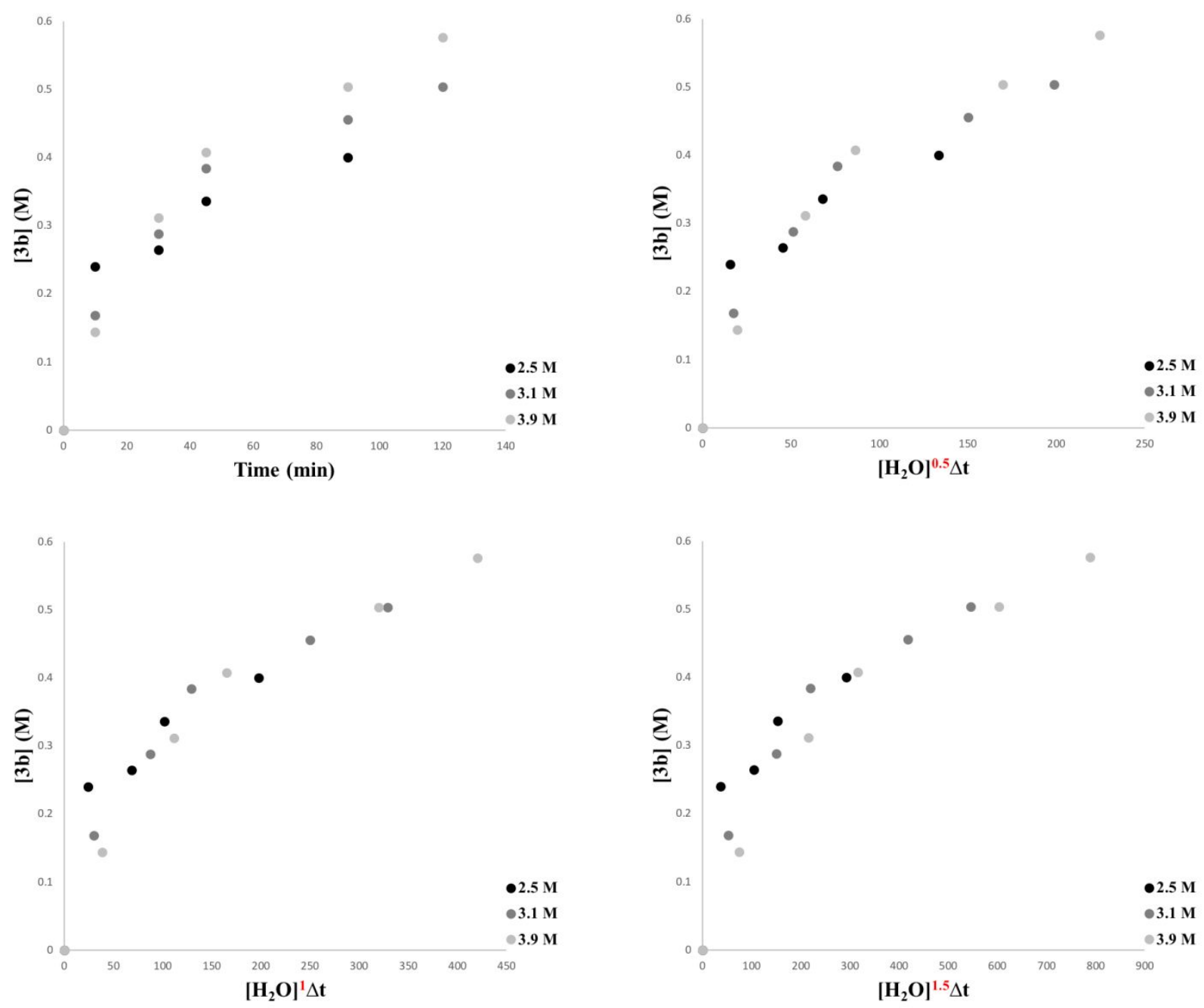

Figure S9. VTNA data indicating optimal overlay using exponent " 1 " for determining the order in $\mathrm{H}_{2} \mathrm{O}$.

It is evident that upon inspection of the VTNA results similar trends are observed. The disparity with respect to the concentration dependences between the two data sets are attributed to competing reaction pathways. In this way, the mechanistic facets of the latter VTNA experiments are reminiscent of the former studies, e.g., positive vs. negative order of components even though the latter kinetic studies have a shortage of starting material components as a result of increased side product formation. 


\section{ii. $\quad$ Kinetic Solvent Isotope Effect Studies}
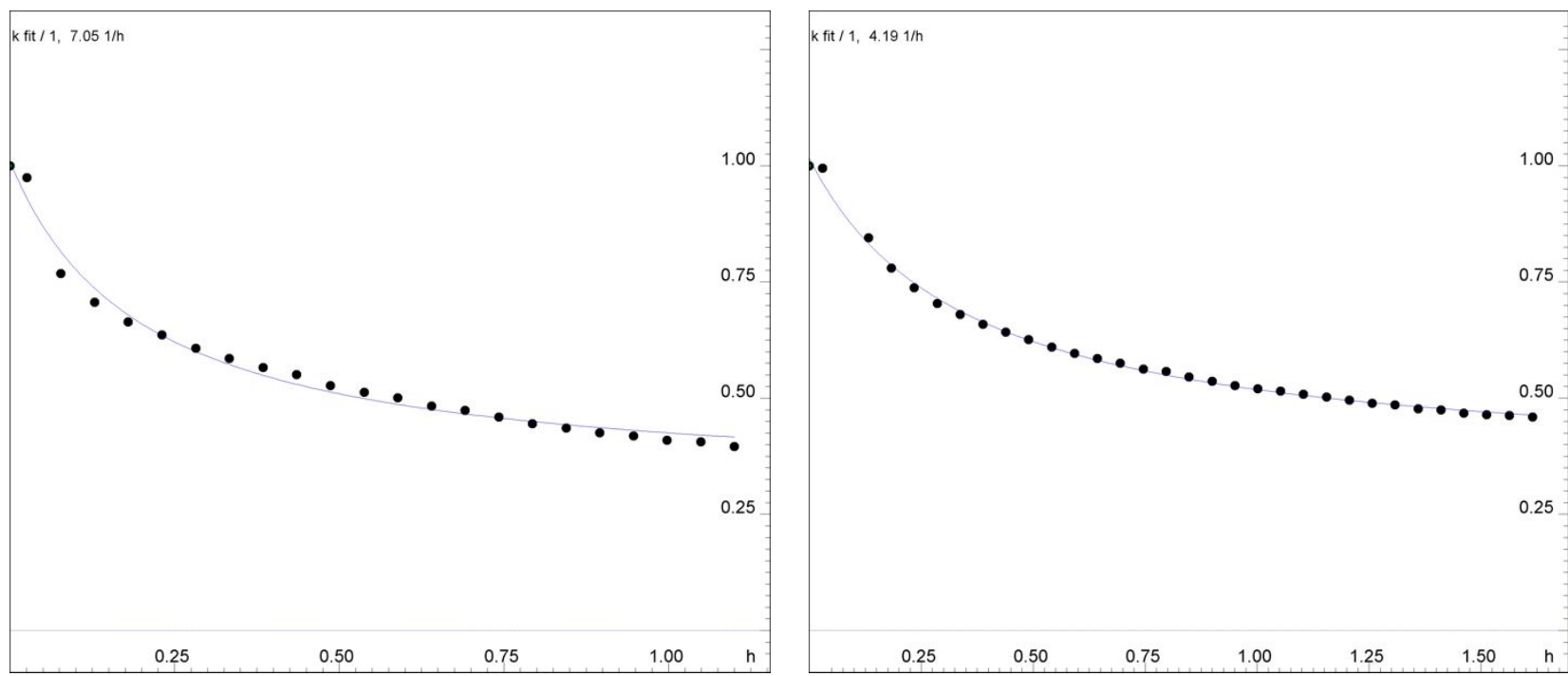

Figure S10. Plots of time (h) vs. signal intensity showcasing rate data using 1a, 2a and $\mathrm{H}_{2} \mathrm{O}$ as substrates with a rate constant, $k$, value of $7.05 \mathrm{~h}^{-1}$ (left-hand side), and 1a, 2a and $\mathrm{D}_{2} \mathrm{O}$ as substrates with a rate constant, $k$, value of $4.19 \mathrm{~h}^{-1}$ (right-hand side). The plots were generated using Bruker's Dynamics Center program.

\section{Experimental Evidence Supporting CSA-Mediated Catalysis}

\section{Procedure for Addition of $\mathrm{D}_{\mathbf{2}} \mathrm{O}$ to DHP in the Presence of Deuterated CSA.}

To initiate this study, CSA $(0.2 \mathrm{~g}, 0.86 \mathrm{mmol})$ was dissolved in $0.78 \mathrm{~mL} \mathrm{D}_{2} \mathrm{O}(43 \mathrm{mmol})$ and stirred for 1 hour at room temperature in a 4 dram vial. Next, the solution was concentrated in vacuo and the process repeated once more. The deuterium content of the catalyst was verified with HRMS (EI) measurement, which was indicative of the majority of compounds being singly deuterated (S2) (exchange with proton on sulfonic acid moiety), with a detectable amount of doubly deuterated (S3) CSA; however, to a lesser extent (Scheme S3). HRMS (EI) m/z: $[\mathrm{M}+\mathrm{H}]^{+}$calcd for $\mathrm{C}_{10} \mathrm{H}_{15} \mathrm{DO}_{4} \mathrm{~S}, 233.0832$; found: 233.0827, and 234.0892.

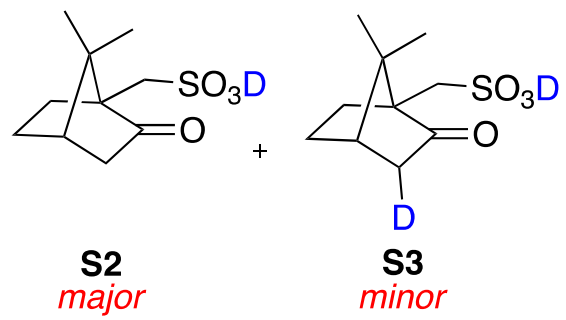

Figure S11. Likely Representation of Deuterated CSA Determined by Mass Spectrometry. 
With deuterated CSA in hand, the reaction forming hydration side product $\mathbf{5 b}$ was pursued and its product distribution probed. Using this simplified system would allow one to confirm the catalytic origin that governs Brønsted acid catalysis. This involved adding deuterated CSA (4 mol\%), and $\mathrm{D}_{2} \mathrm{O}(1.8 \mathrm{mmol})$, respectively to a stirring solution of DHP $(1.2 \mathrm{mmol})$ in $0.5 \mathrm{~mL}$ THF using a 4 dram vial. The reaction was stirred for $4 \mathrm{~h}$. Upon analysis of the HRMS (EI) measurement, only singly deuterated hydration product was detected. HRMS (EI) m/z: $[\mathrm{M}+\mathrm{H}]^{+}$calcd for $\mathrm{C}_{5} \mathrm{H}_{8} \mathrm{D}_{2} \mathrm{O}_{2}$, 104.0806; found: 102.0664 .<smiles>[2H]C1CCCOC1[O-]</smiles>

S4

Figure S12. Likely Compound Correlating to the Mass Value Obtained from Mass Spectrometry.

Scheme S3. A) Addition of $\mathrm{D}_{2} \mathrm{O}$ to DHP using Deuterated CSA. B) Reversible vs. irreversible nucleophilic addition mechanisms mediated by either hydronium or CSA, respectively.

A

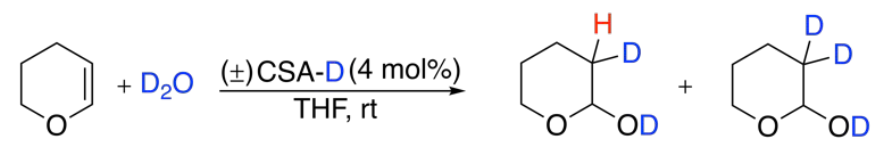

2b

$5 b^{\prime}$

5b"

Hydronium-catalyzed
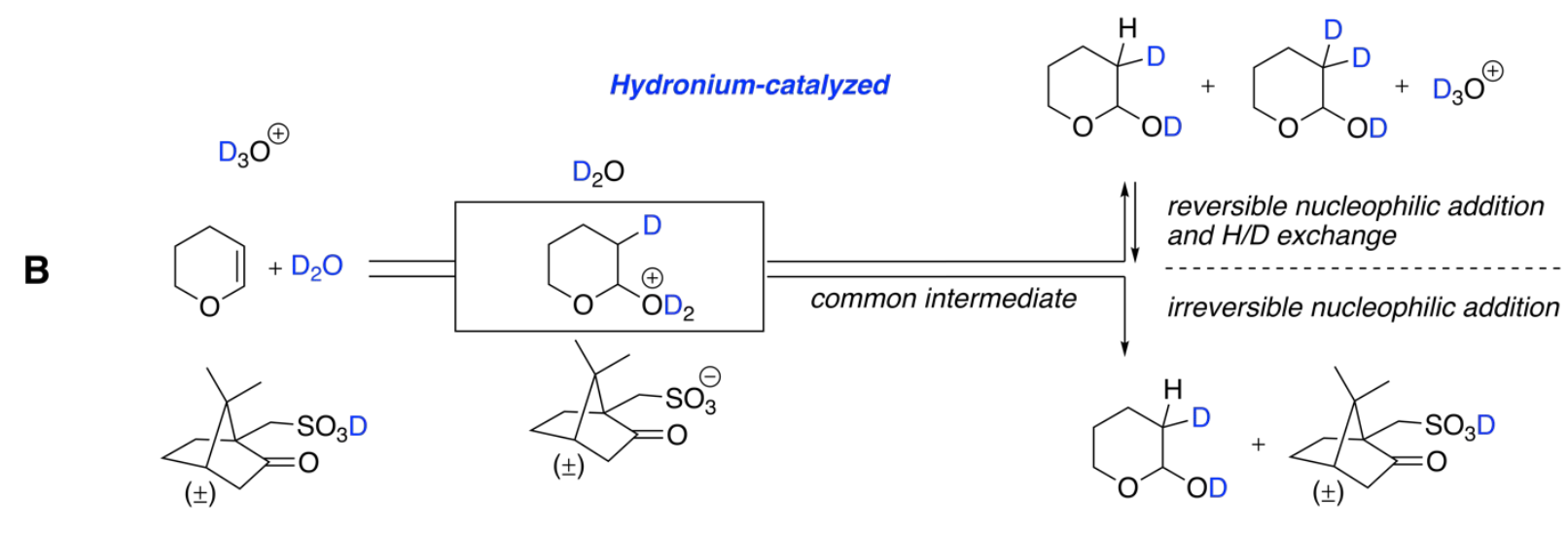

CSA-catalyzed 


\section{Spectra}

$\underline{{ }^{1} \mathrm{H} \mathrm{NMR}(300 \mathrm{MHz}) \text { Spectrum of 3a in } \mathrm{CDCl}_{3}}$

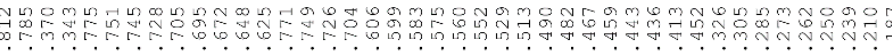

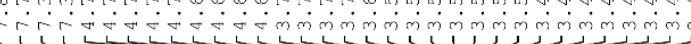

$\overbrace{0}^{N} \overbrace{0}^{H}{ }^{T o s}$

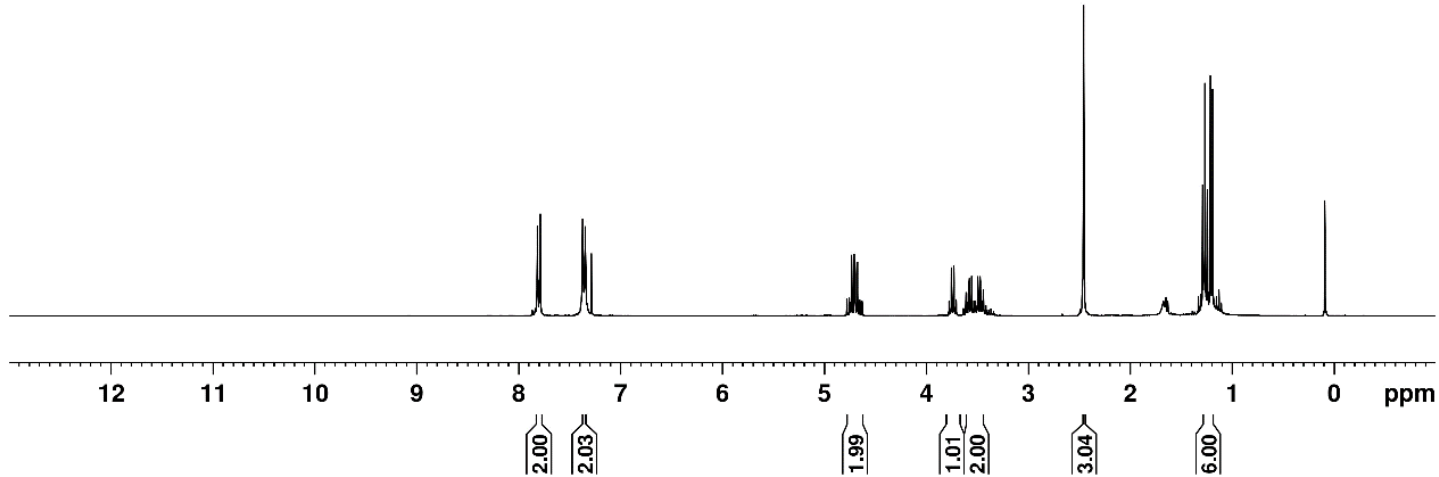

${ }^{13} \mathrm{C}\left\{{ }^{1} \mathrm{H}\right\}$ NMR (75.5 MHz) Spectrum of 3a in $\mathrm{CDCl}_{3}$

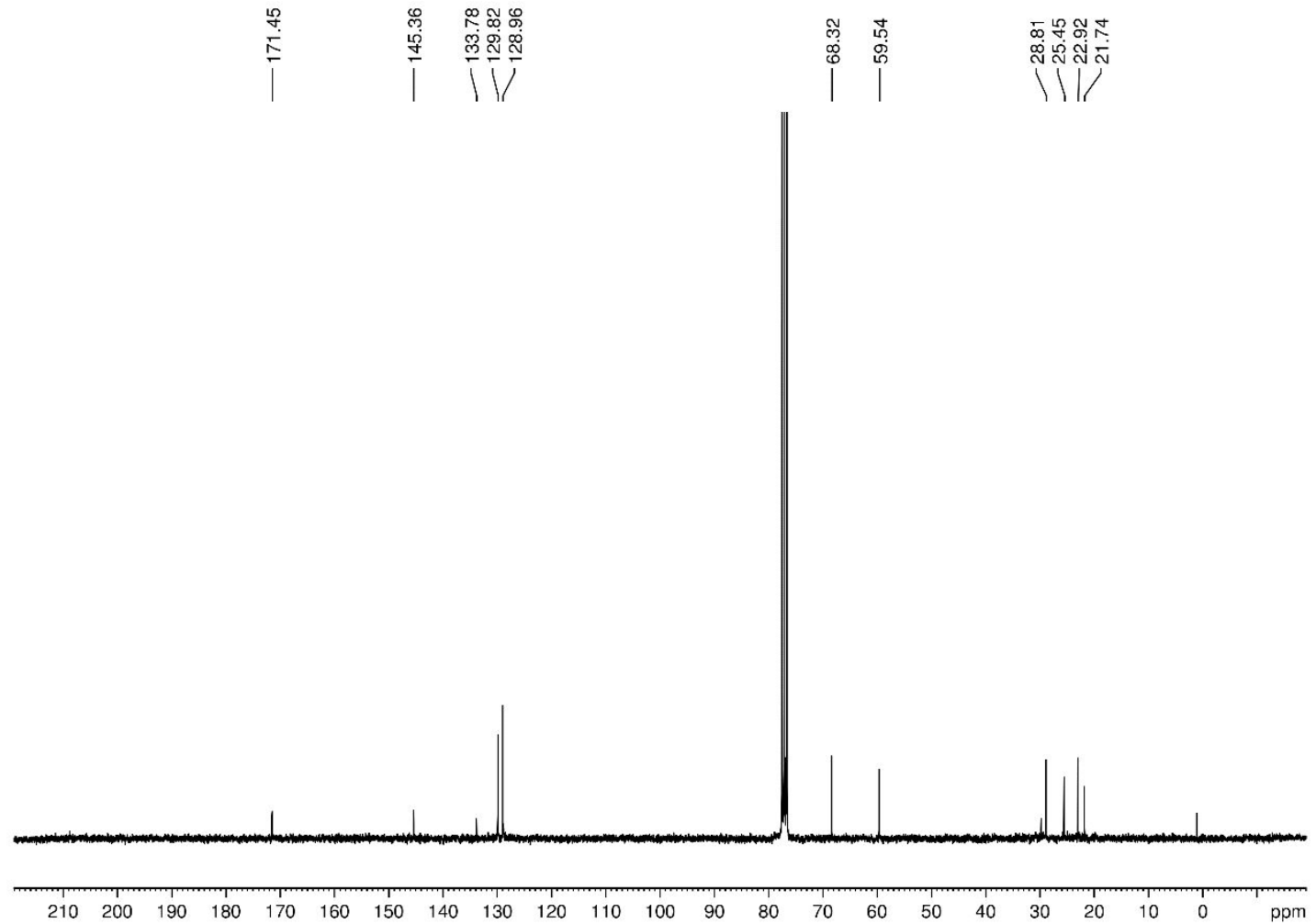


$\underline{{ }^{1} \mathrm{H} \text { NMR (300 MHz) Spectrum of } \mathbf{3 b} \text { in } \mathrm{CDCl}_{3}}$

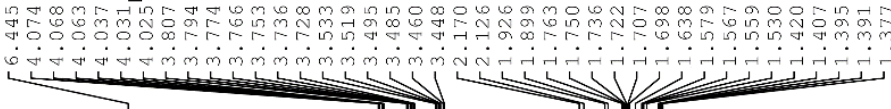<smiles>O=C(NC1CCCCC1)C1CCCCO1</smiles>

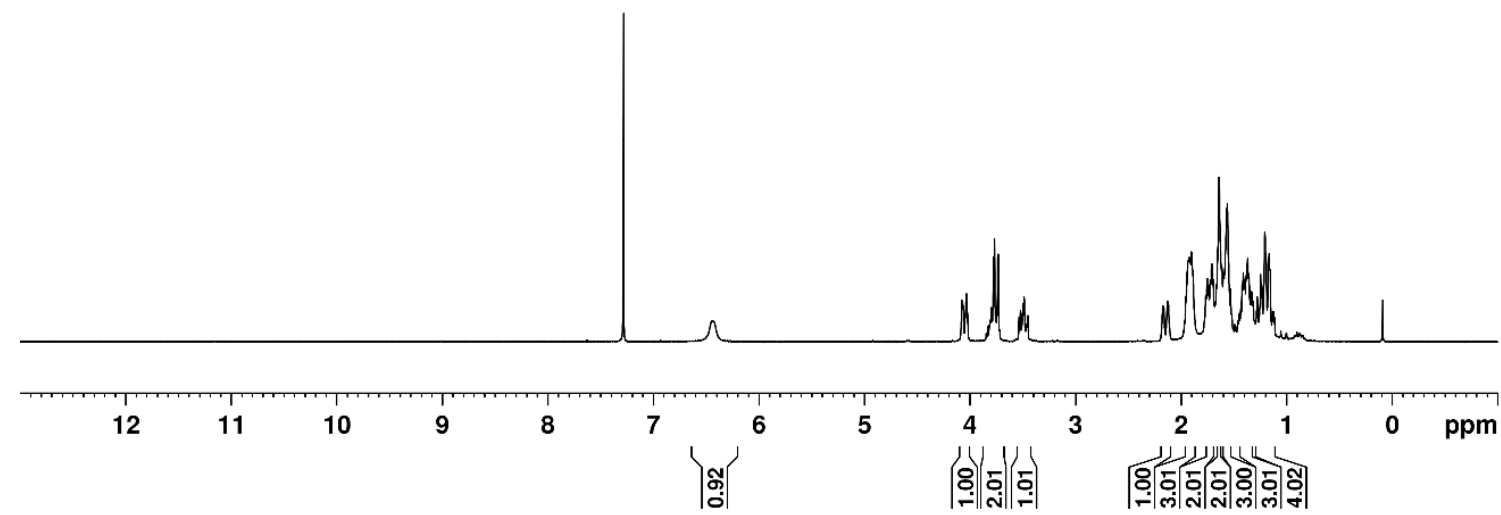

${ }^{13} \mathrm{C}\left\{{ }^{1} \mathrm{H}\right\} \mathrm{NMR}(75.5 \mathrm{MHz})$ Spectrum of $\mathbf{3 b}$ in $\mathrm{CDCl}_{3}$
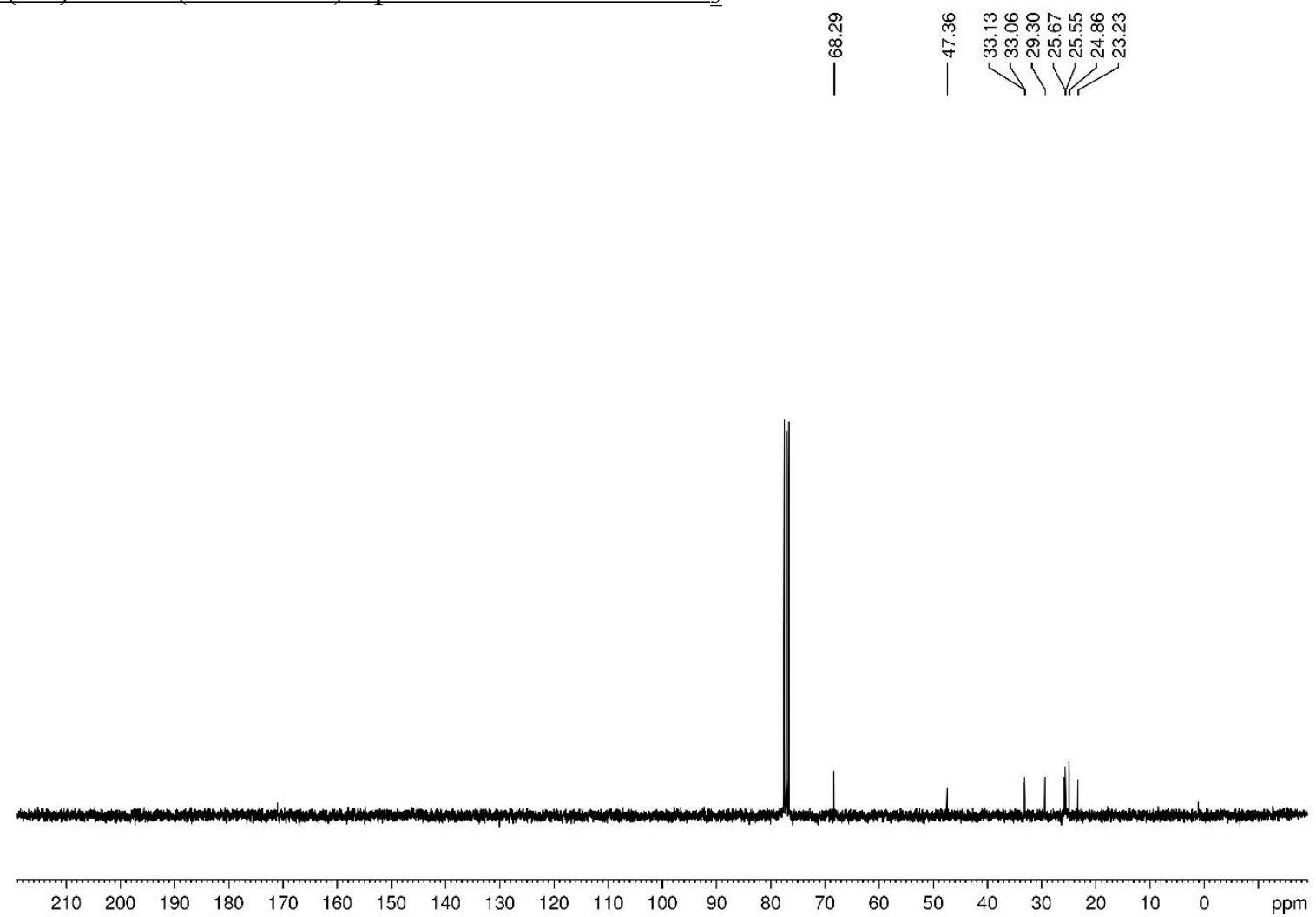
$\underline{{ }^{1} \mathrm{H} \text { NMR (300 MHz) Spectrum of } \mathbf{3 c} \text { in } \mathrm{CDCl}_{3}}$

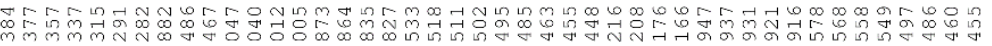
$\therefore$ -<smiles>O=C(NCc1ccccc1)C1CCCCO1</smiles>

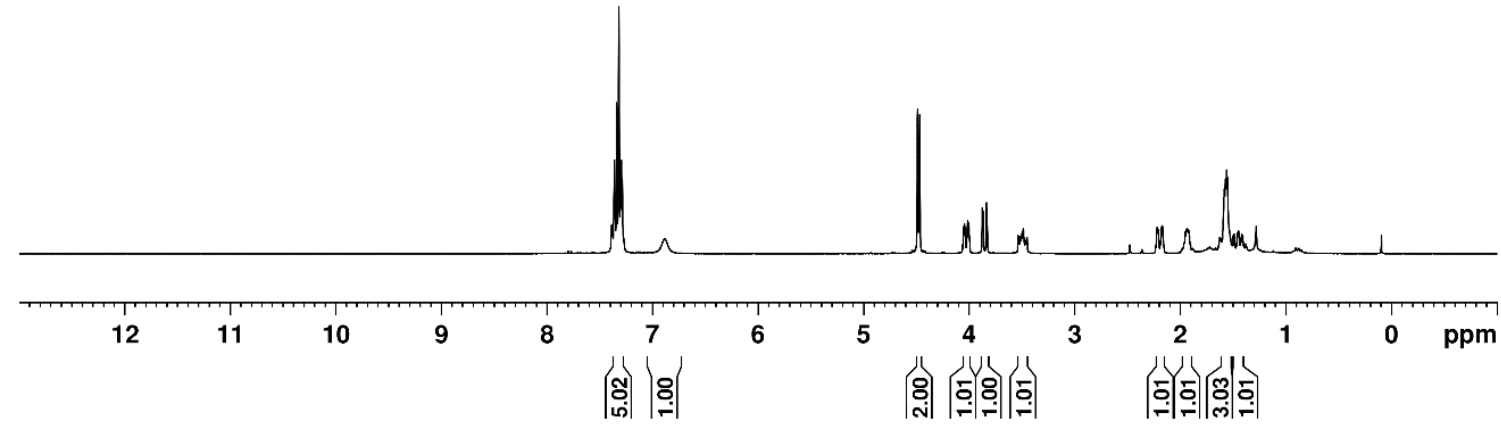

${ }^{13} \mathrm{C}\left\{{ }^{1} \mathrm{H}\right\}$ NMR (75.5 MHz) Spectrum of $3 \mathbf{c}$ in $\mathrm{CDCl}_{3}$
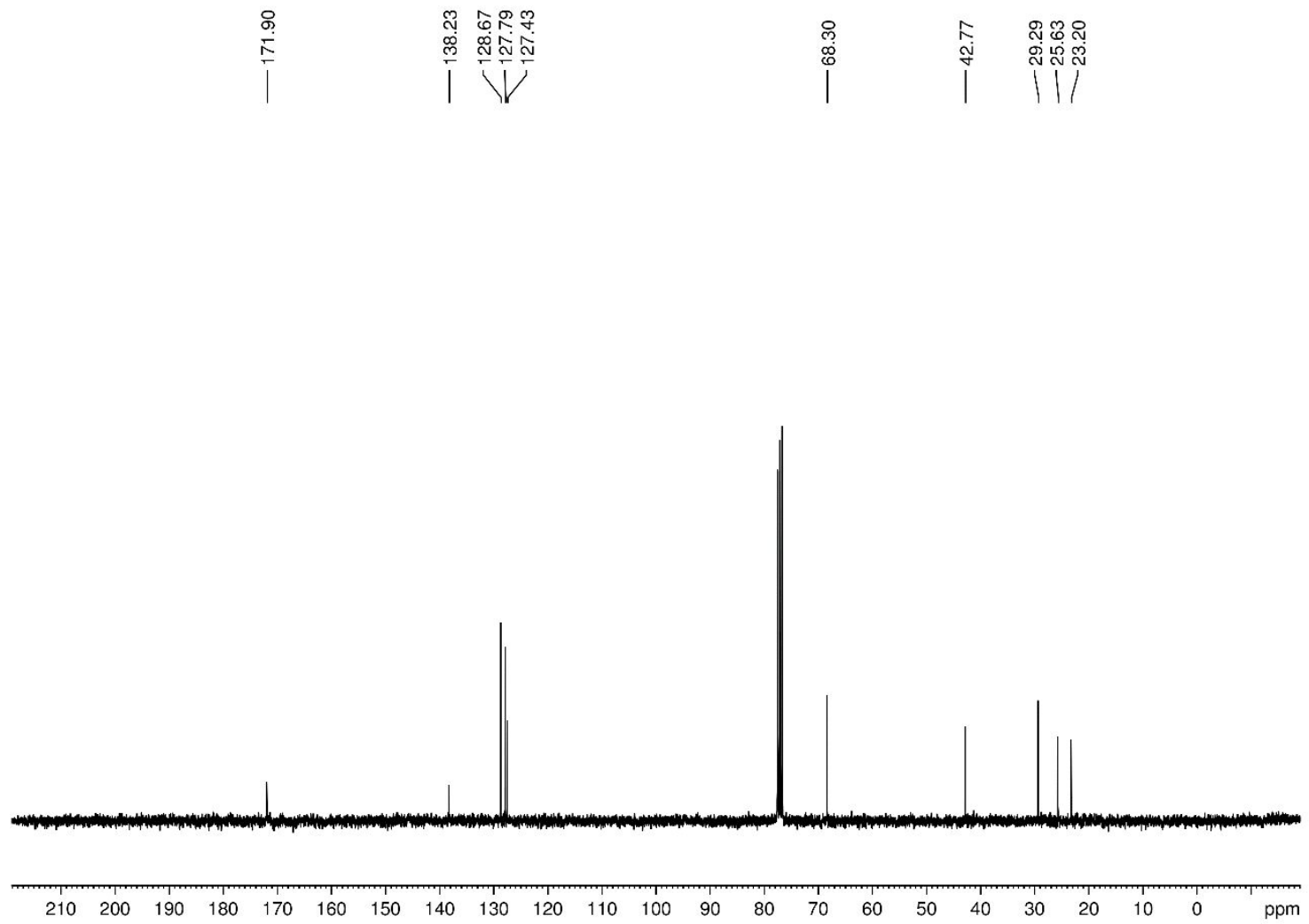
$\underline{{ }^{1} \mathrm{H} N M R}$ (300 MHz) Spectrum of $\mathbf{3 d}$ in $\mathrm{CDCl}_{3}$

VV

$\overbrace{0}^{H} \sim^{\text {Tos }}$
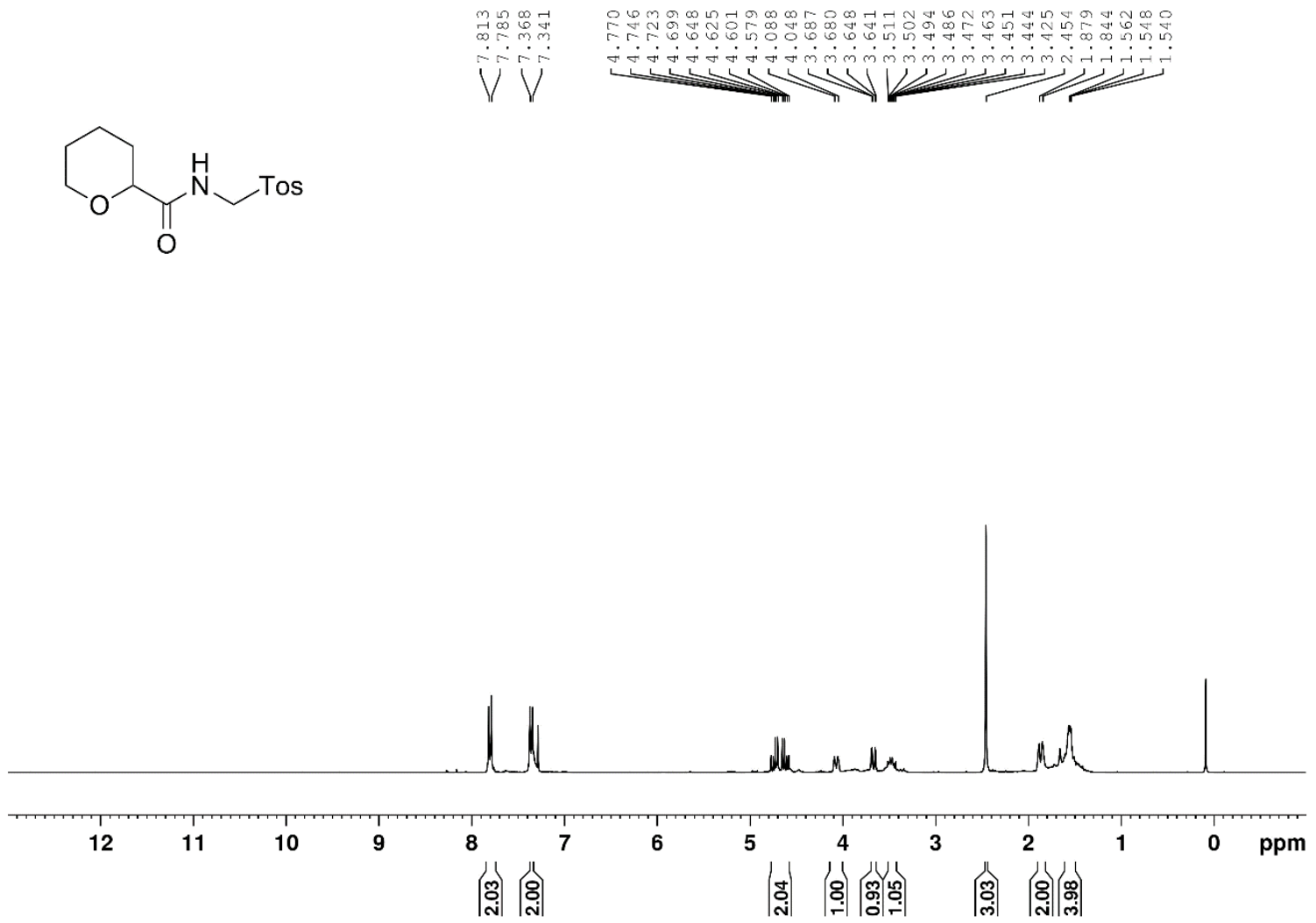

$\underline{{ }^{13} \mathrm{C}\left\{{ }^{1} \mathrm{H}\right\} \text { NMR (75.5 MHz) Spectrum of } \mathbf{3 d} \text { in } \mathrm{CDCl}_{3}}$
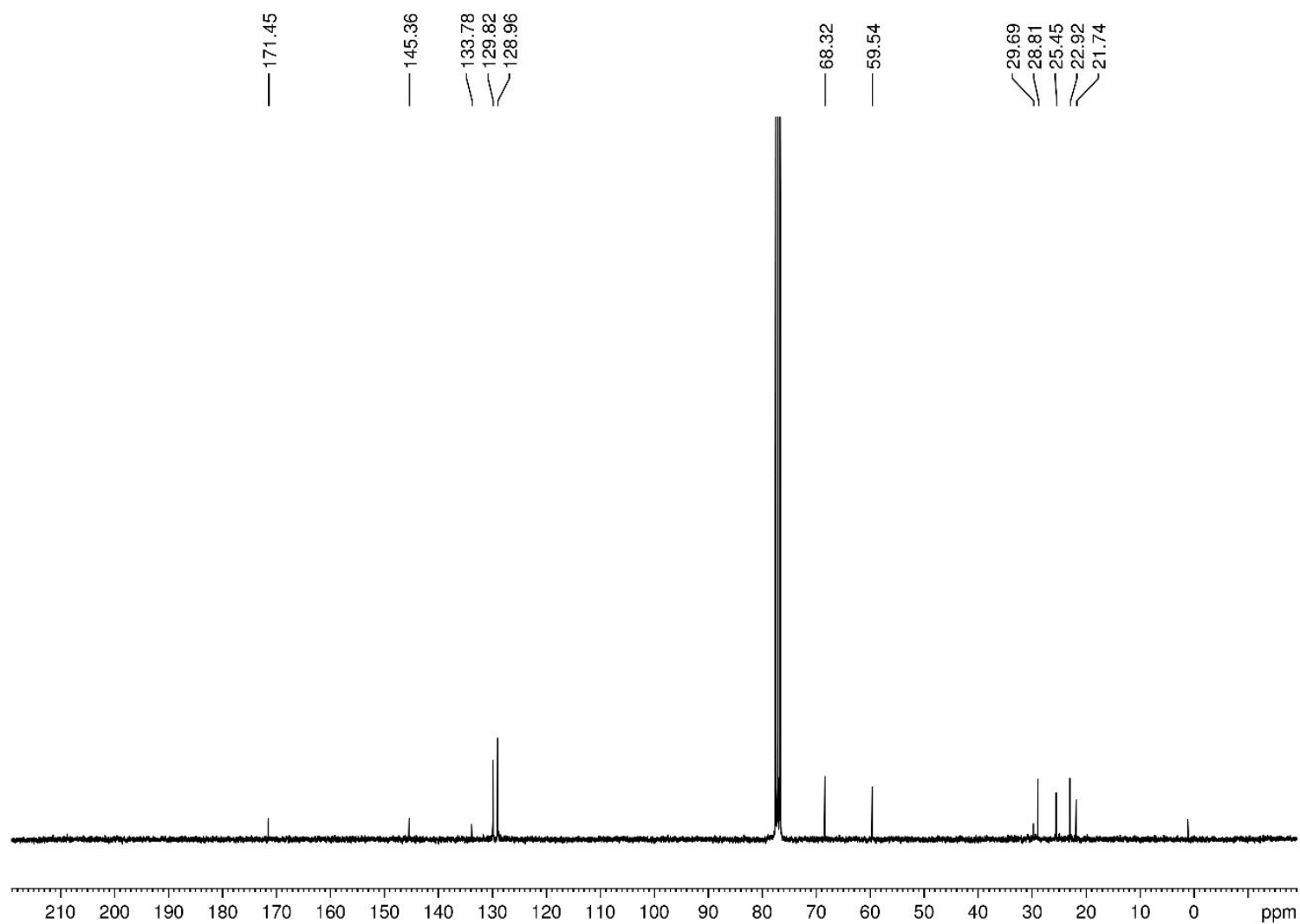
$\underline{{ }^{1} \mathrm{H} N M R}(300 \mathrm{MHz})$ Spectrum of $\mathbf{3 e}$ in $\mathrm{CDCl}_{3}$

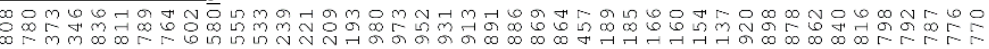

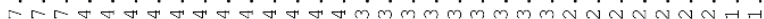

$\overbrace{0} \overbrace{0}^{H} \sim^{T o s}$

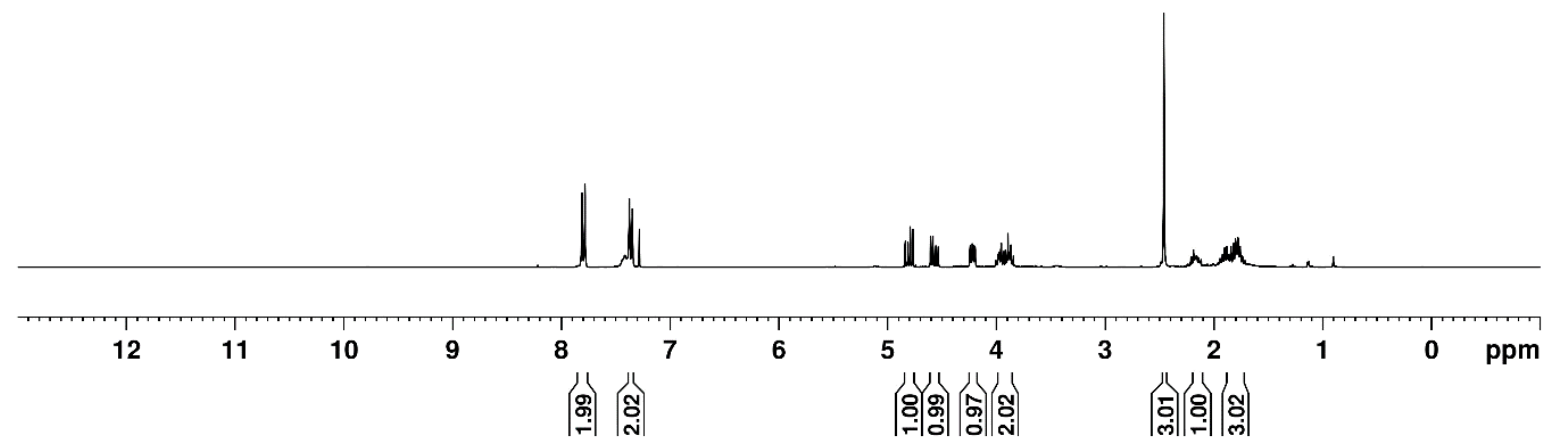

${ }^{13} \mathrm{C}\left\{{ }^{1} \mathrm{H}\right\}$ NMR (75.5 MHz) Spectrum of $3 \mathbf{e}$ in $\mathrm{CDCl}_{3}$
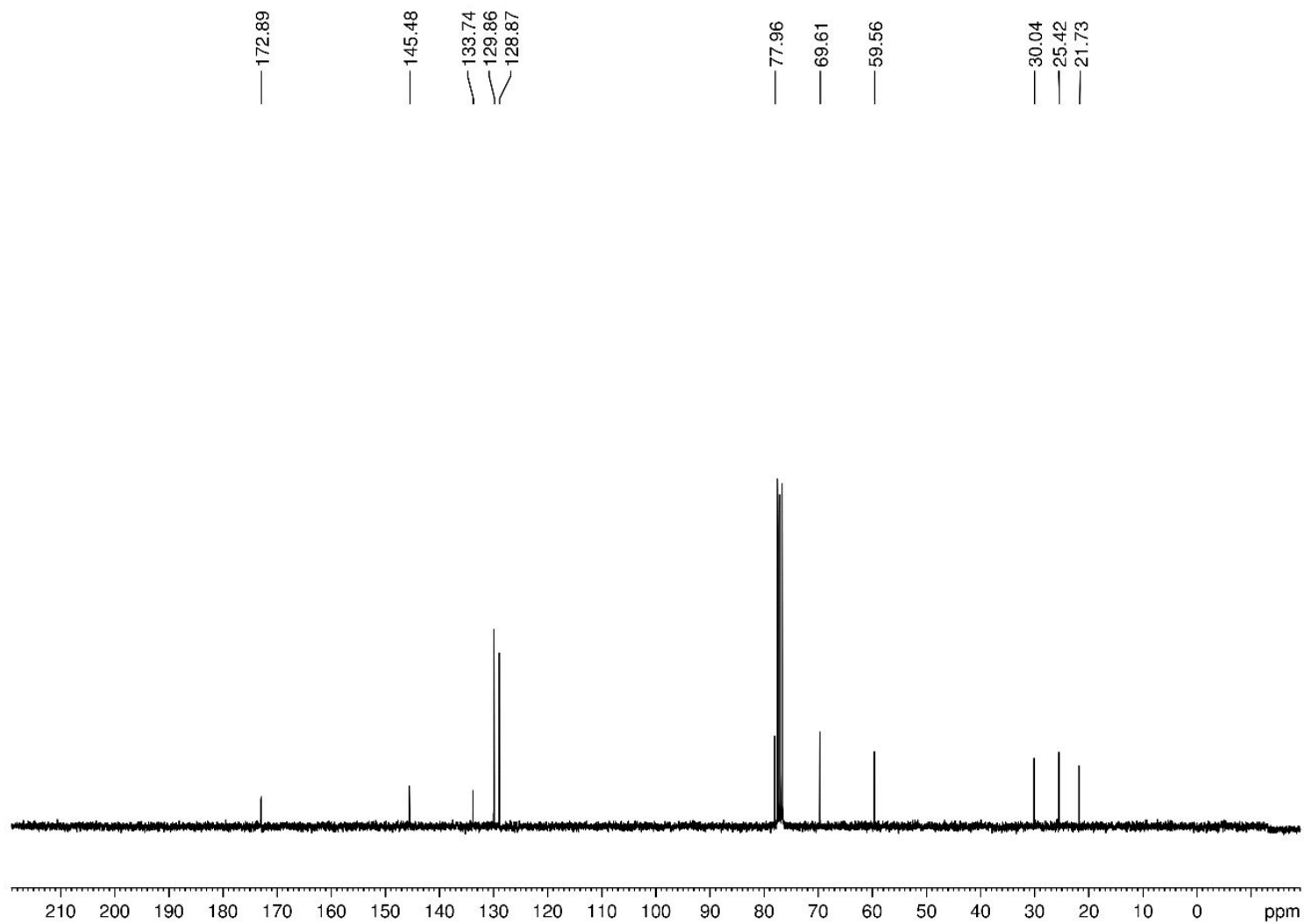
$\underline{{ }^{1} \mathrm{H} \text { NMR (300 MHz) Spectrum of } \mathbf{3} \mathbf{f} \text { in } \mathrm{CDCl}_{3}}$

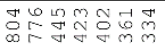<smiles>CC(NCC(=O)OC1CCCCC1)C(=O)OC1CCCCC1</smiles>

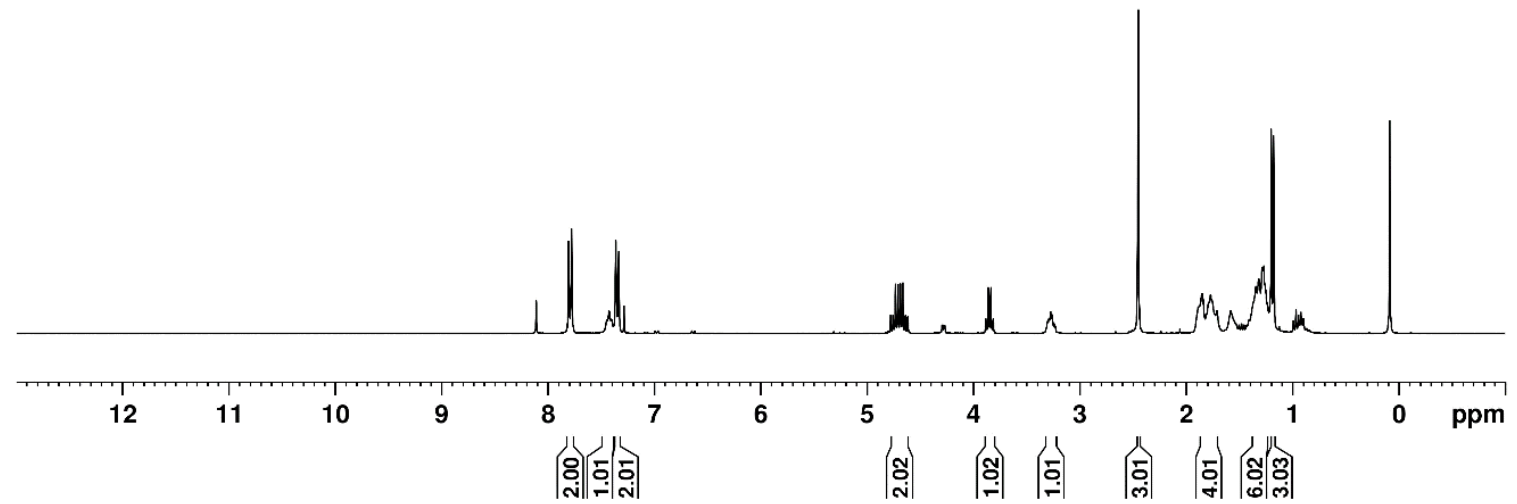

${ }^{13} \mathrm{C}\left\{{ }^{1} \mathrm{H}\right\}$ NMR (75.5 MHz) Spectrum of $\mathbf{3 f}$ in $\mathrm{CDCl}_{3}$

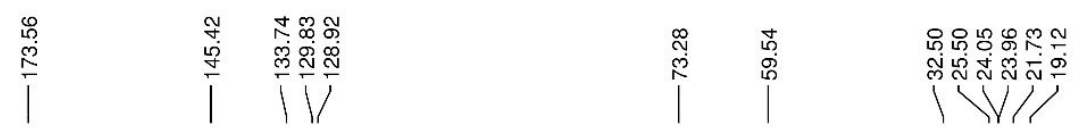
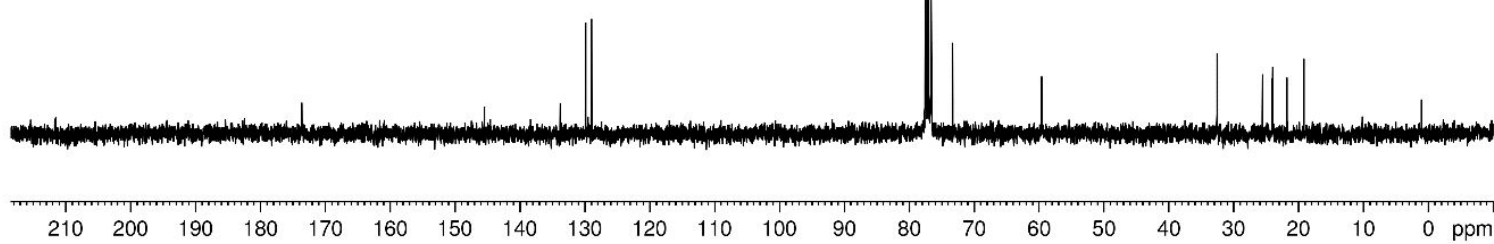
$\underline{{ }^{1} \mathrm{H} \text { NMR (300 MHz) Spectrum of } \mathbf{3 g} \text { in } \mathrm{CDCl}_{3}}$

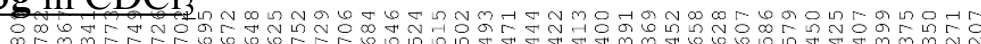

14 -<smiles>CCCCOC(C)C(=O)NCC(=O)[O-]</smiles>

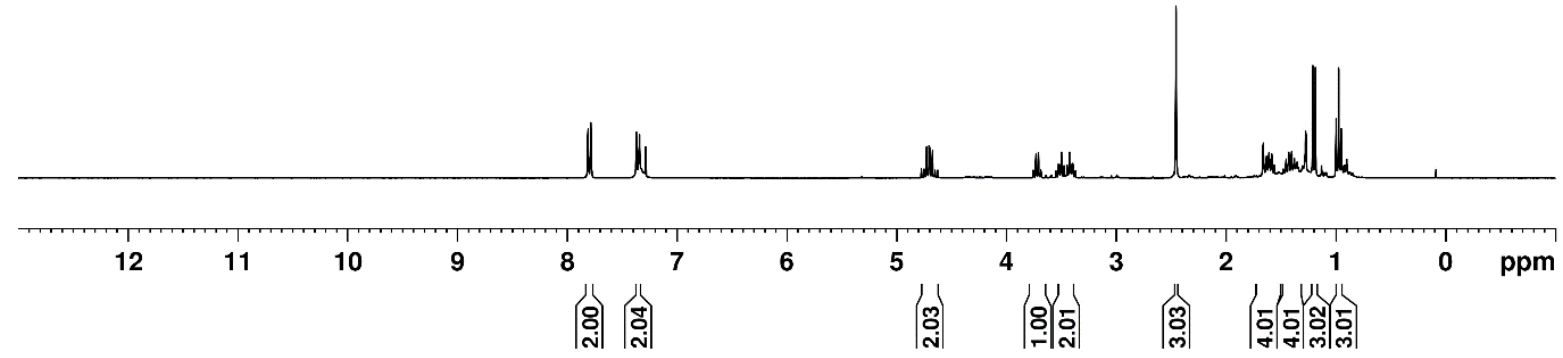

${ }^{13} \mathrm{C}\left\{{ }^{1} \mathrm{H}\right\}$ NMR (75.5 MHz) Spectrum of $\mathbf{3 g}$ in $\mathrm{CDCl}_{3}$

| |

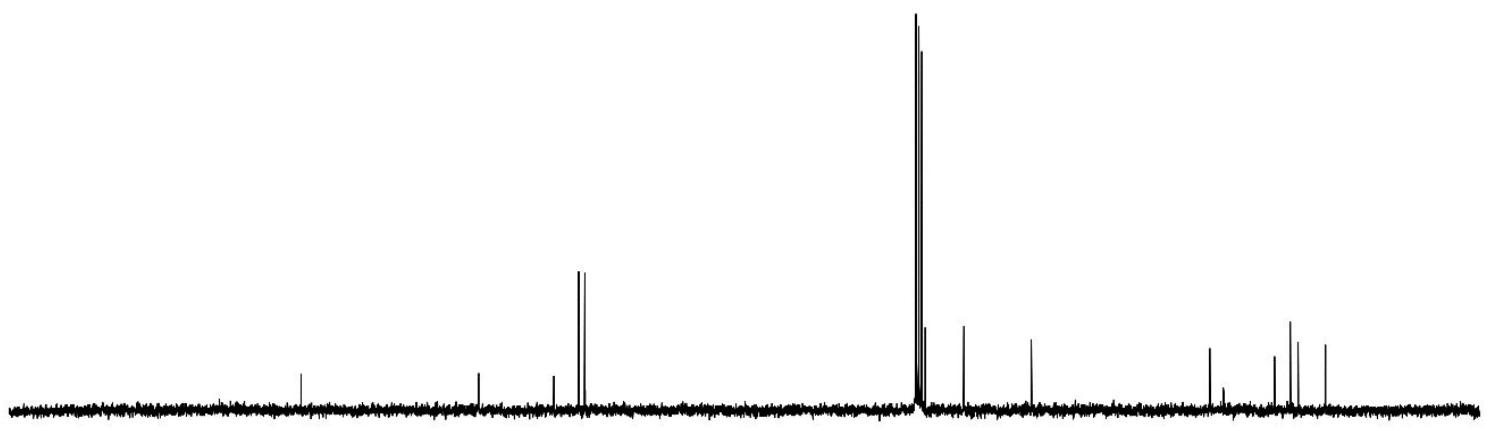

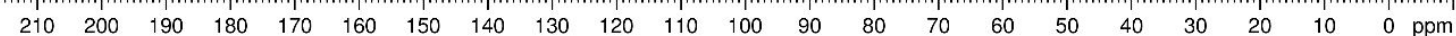


$\underline{{ }^{1} \mathrm{H} \text { NMR (300 MHz) Spectrum of } \mathbf{3 h} \text { in } \mathrm{CDCl}_{3}}$

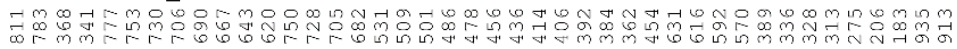

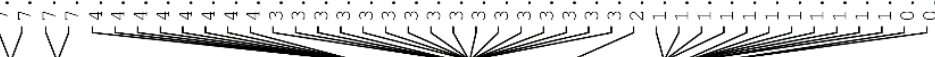

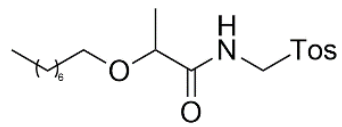

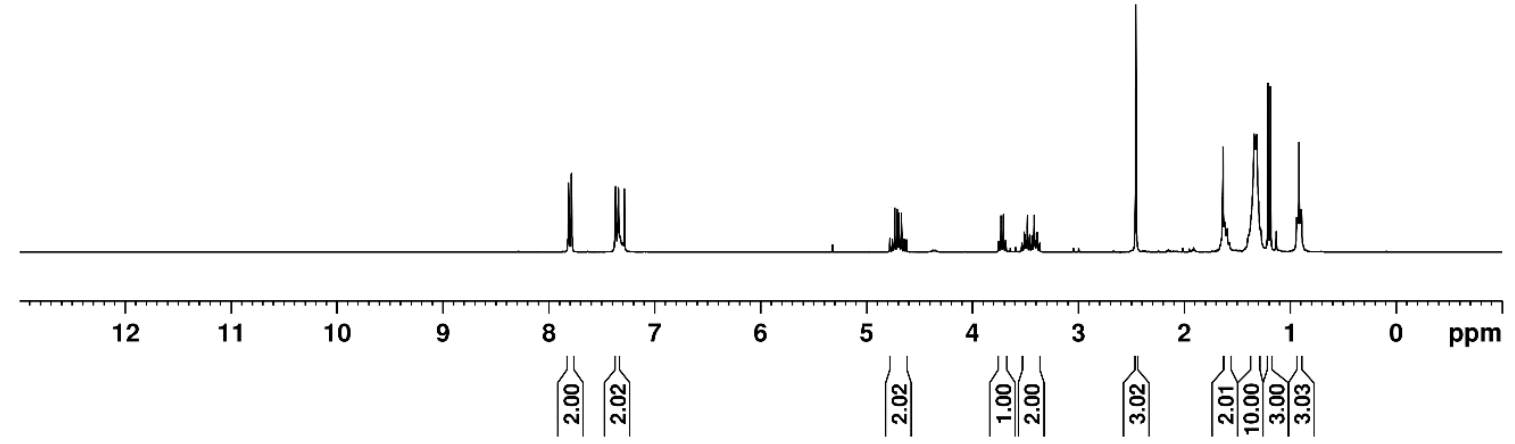

${ }^{13} \mathrm{C}\left\{{ }^{1} \mathrm{H}\right\}$ NMR (75.5 MHz) Spectrum of $\mathbf{3 h}$ in $\mathrm{CDCl}_{3}$

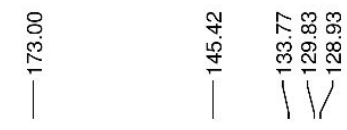

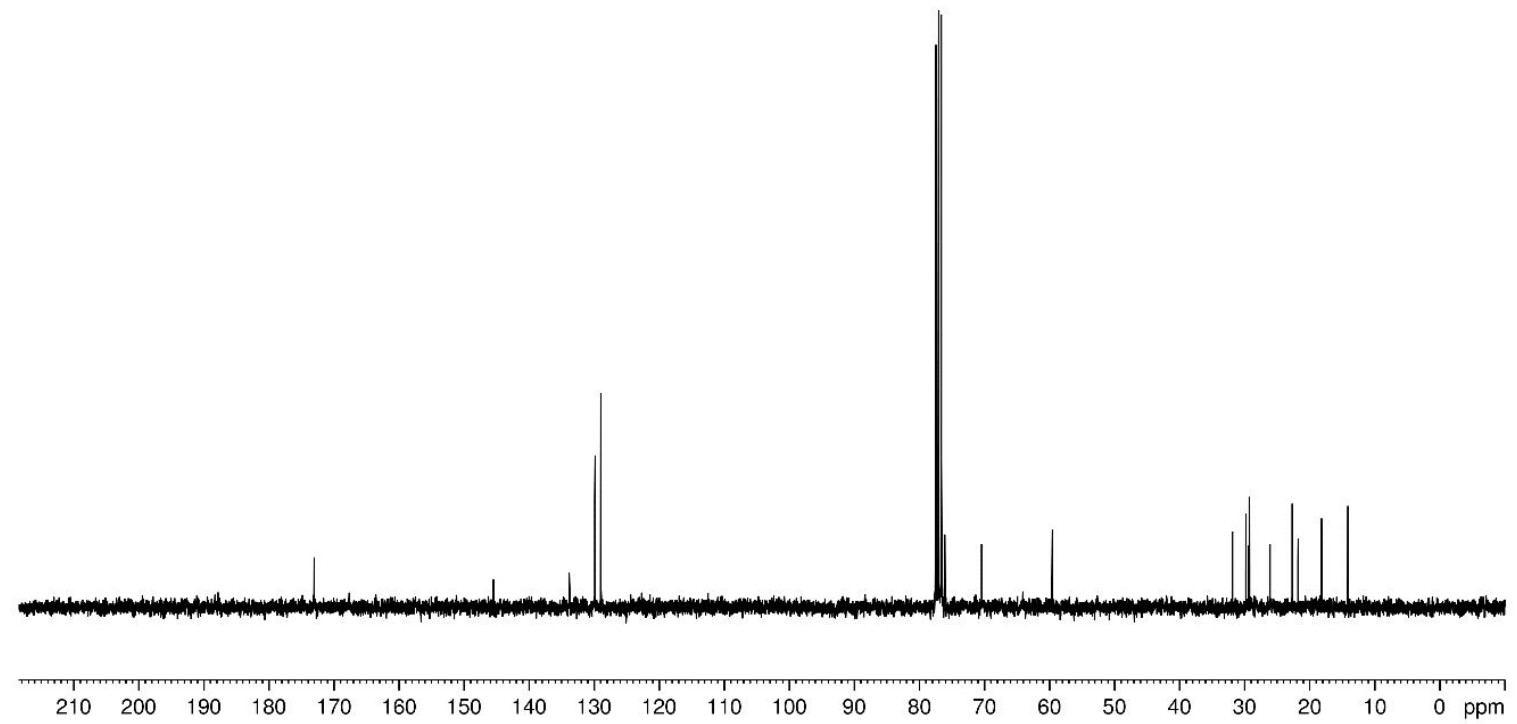


$\underline{{ }^{1} \mathrm{H} N M R}(300 \mathrm{MHz})$ Spectrum of $3 \mathbf{i}$ in $\mathrm{CDCl}_{3}$

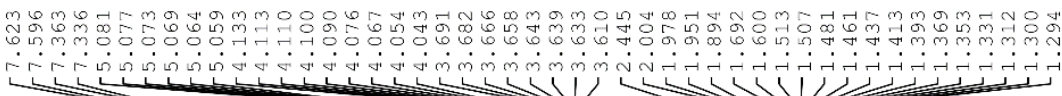<smiles>CC1=CCCC(C)CC(C(=O)NC[Ge])C1</smiles>

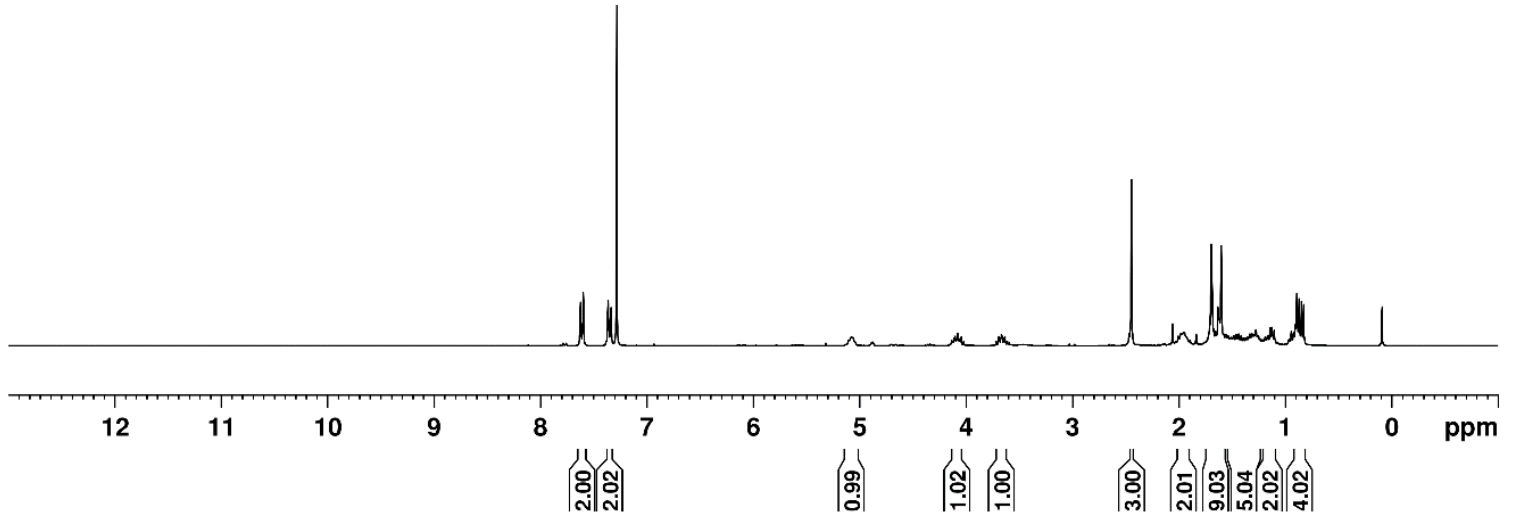

${ }^{13} \mathrm{C}\left\{{ }^{1} \mathrm{H}\right\}$ NMR (75.5 MHz) Spectrum of $3 \mathbf{i}$ in $\mathrm{CDCl}_{3}$

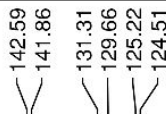

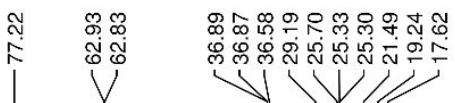
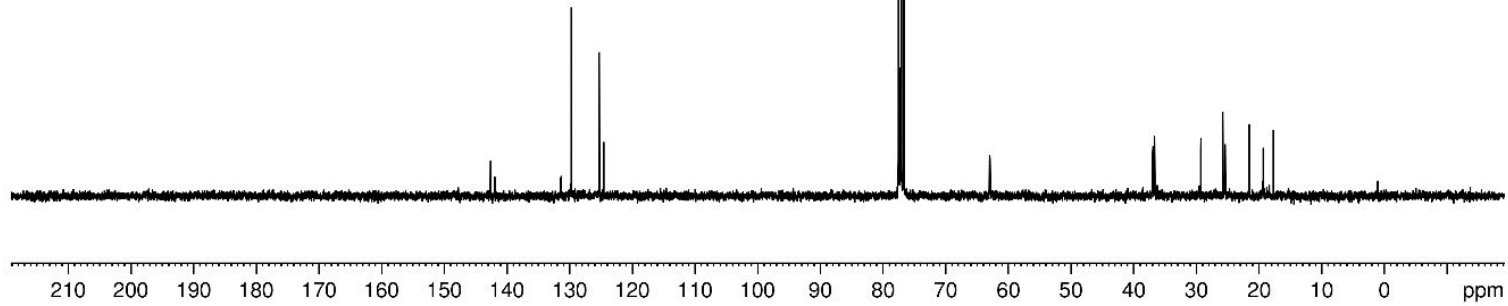
$\underline{{ }^{1} \mathrm{H} \text { NMR (300 MHz) Spectrum of } \mathbf{3} \mathbf{j} \text { in } \mathrm{CDCl}_{3}}$
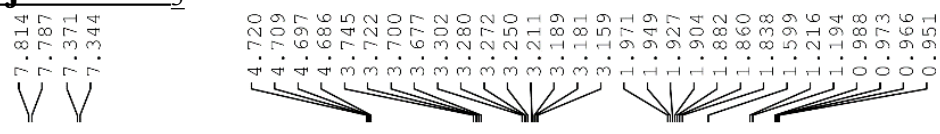

$\gamma_{0} \overbrace{0}^{\mathrm{N}} \sim^{\mathrm{T} o s}$

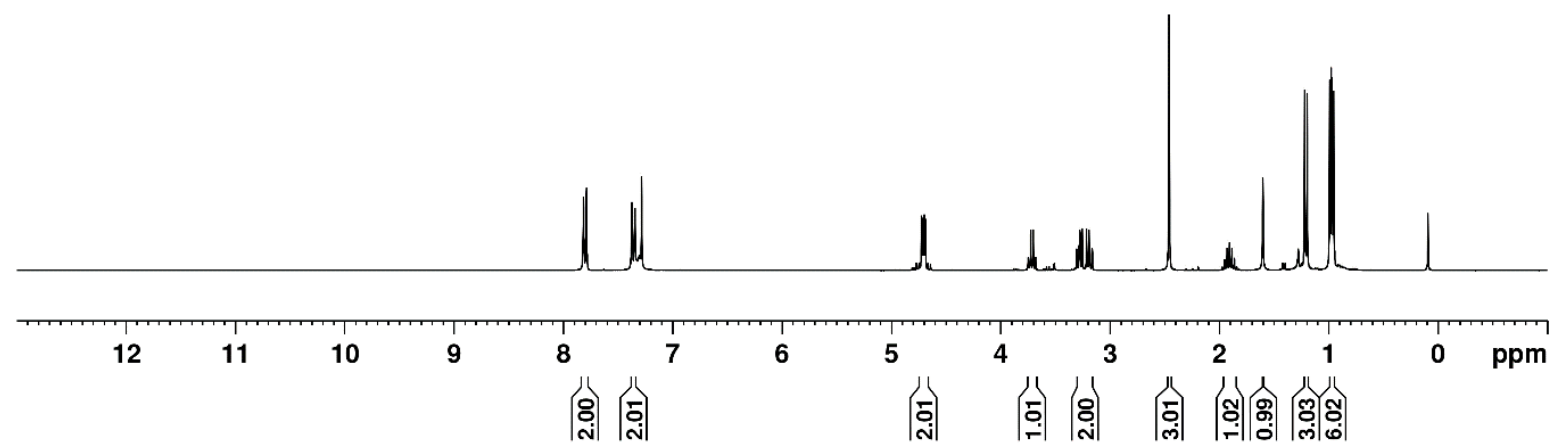

${ }^{13} \mathrm{C}\left\{{ }^{1} \mathrm{H}\right\}$ NMR (75.5 MHz) Spectrum of $\mathbf{3} \mathbf{j}$ in $\mathrm{CDCl}_{3}$

永
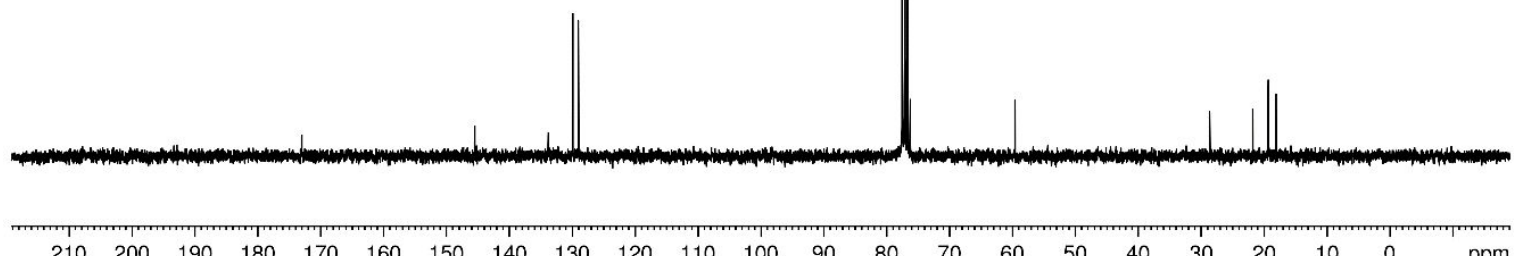
$\underline{{ }^{1} \mathrm{H} \text { NMR (300 MHz) Spectrum of } \mathbf{3 k} \text { in } \mathrm{CDCl}_{3}}$

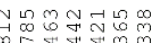

$\chi_{0} \underbrace{\mathrm{H}}_{0} \sim^{\text {Tos }}$

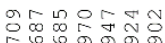

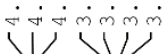

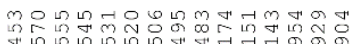

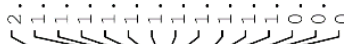

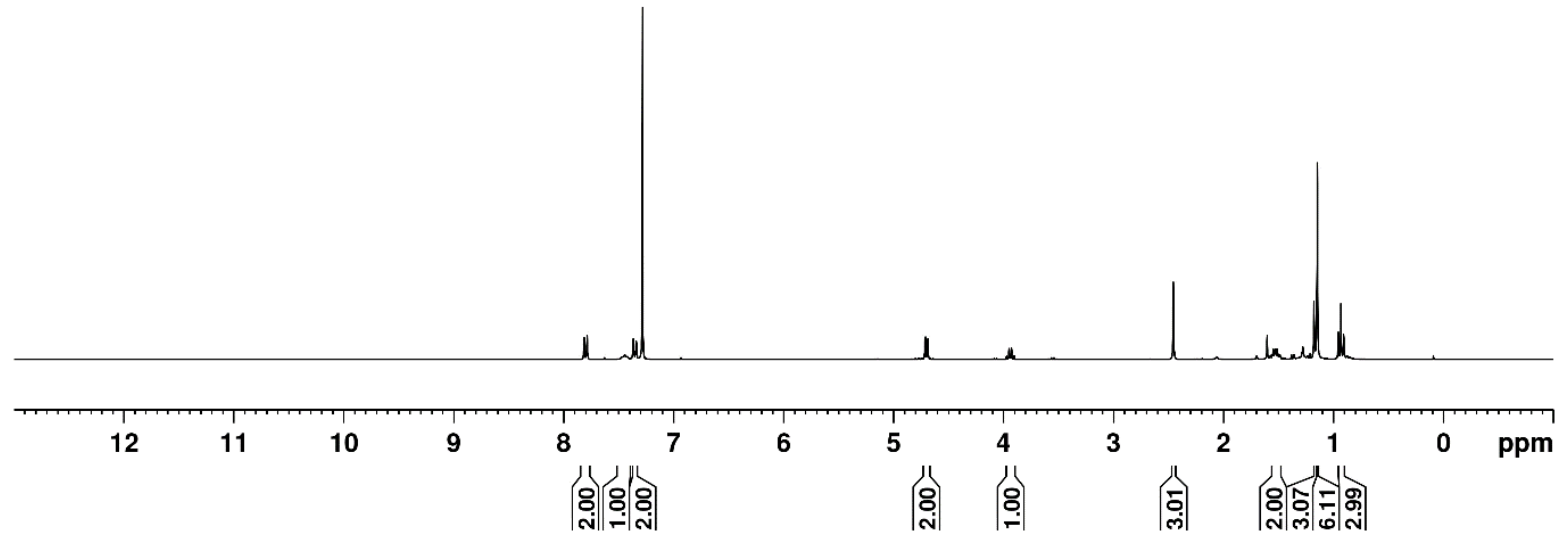

${ }^{13} \mathrm{C}\left\{{ }^{1} \mathrm{H}\right\}$ NMR (75.5 MHz) Spectrum of 3k in $\mathrm{CDCl}_{3}$

|

బ
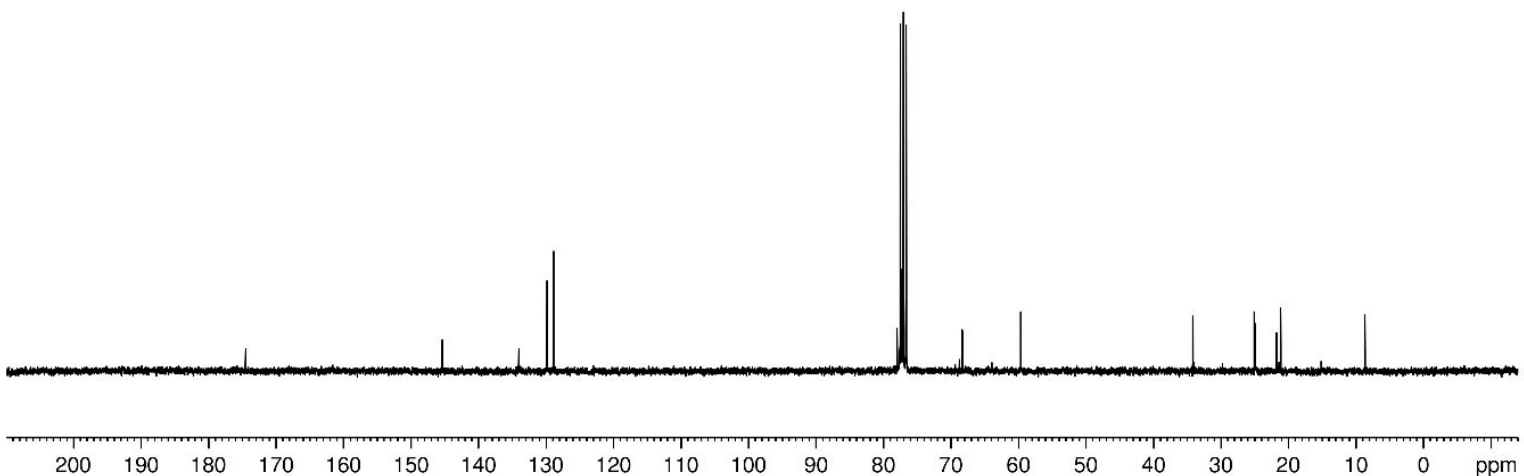
$\underline{{ }^{1} \mathrm{H} \text { NMR (300 MHz) Spectrum of } \mathbf{3 1} \text { in } \mathrm{CDCl}_{3}}$

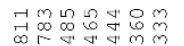

iriver

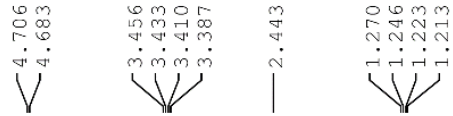

$\sim_{0} \chi_{0}^{\mathrm{H}} \sim^{\text {Tos }}$

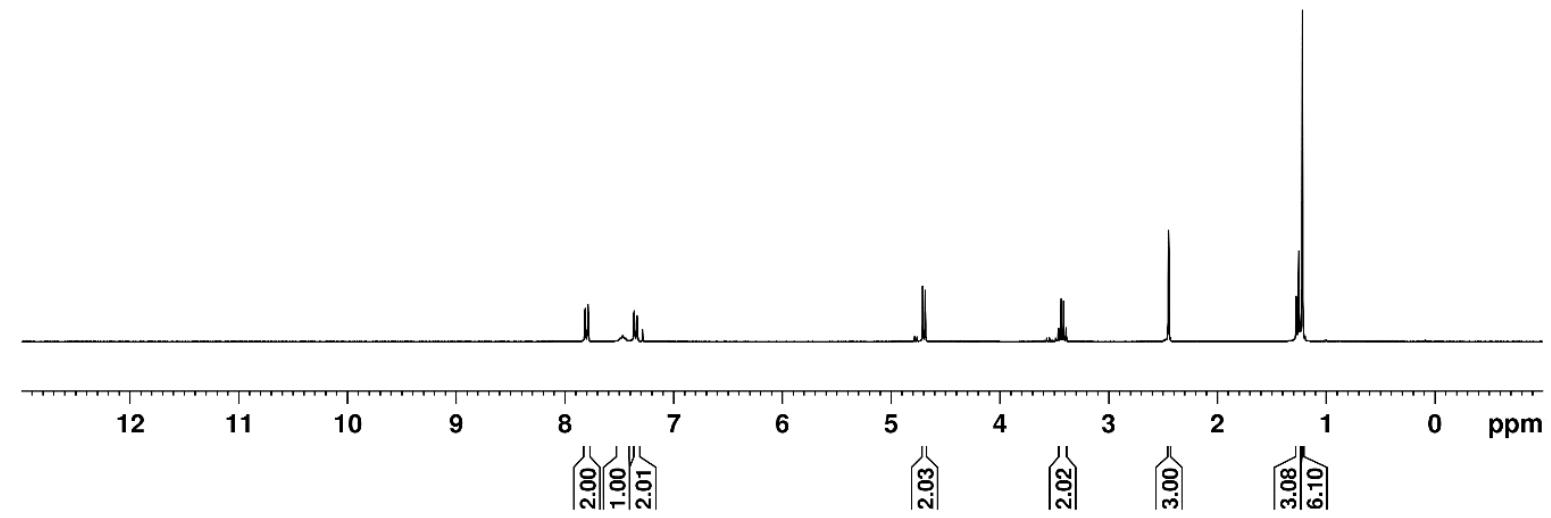

$\underline{{ }^{13} \mathrm{C}\left\{{ }^{1} \mathrm{H}\right\} \text { NMR (75.5 MHz) Spectrum of } 31 \text { in } \mathrm{CDCl}_{3}}$

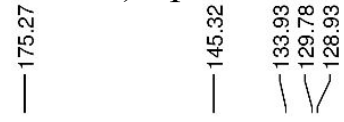

$\left.\right|^{\infty}$
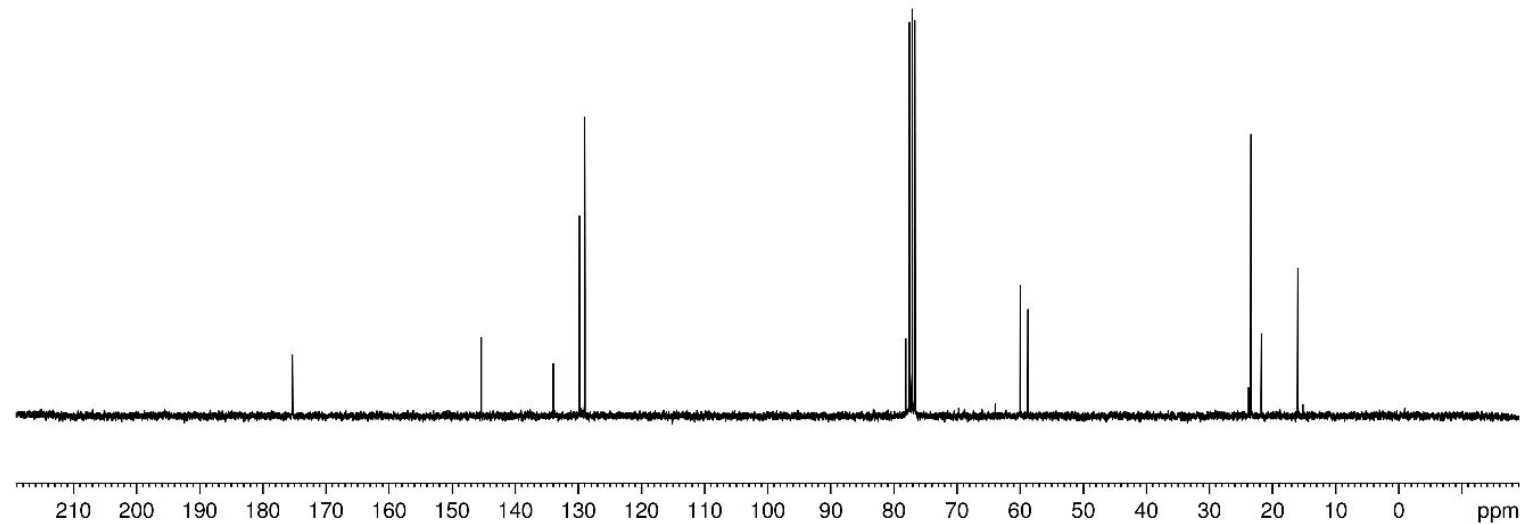
$\underline{{ }^{1} \mathrm{H} N M R}(300 \mathrm{MHz})$ Spectrum of 4 in $\mathrm{CDCl}_{3}$<smiles></smiles>

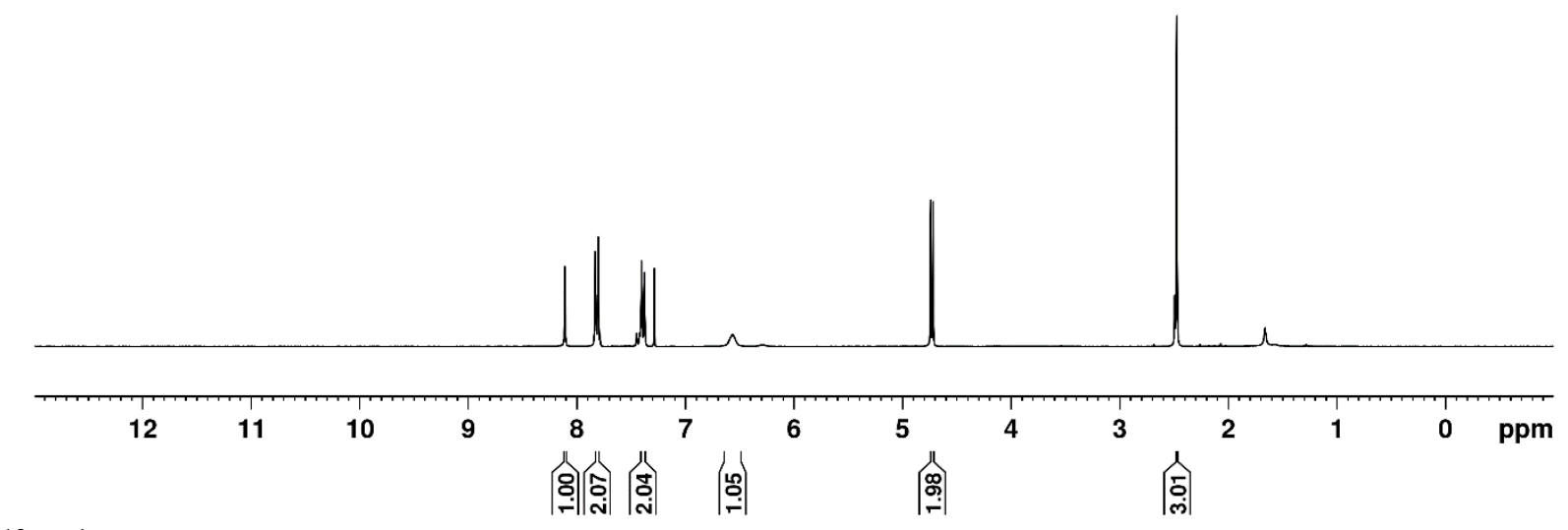

$\underline{{ }^{13} \mathrm{C}\left\{{ }^{1} \mathrm{H}\right\} \text { NMR (75.5 MHz) Spectrum of } 4 \text { in } \mathrm{CDCl}_{3}}$

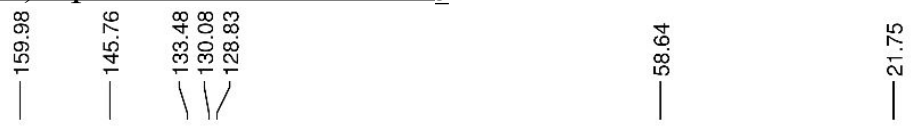
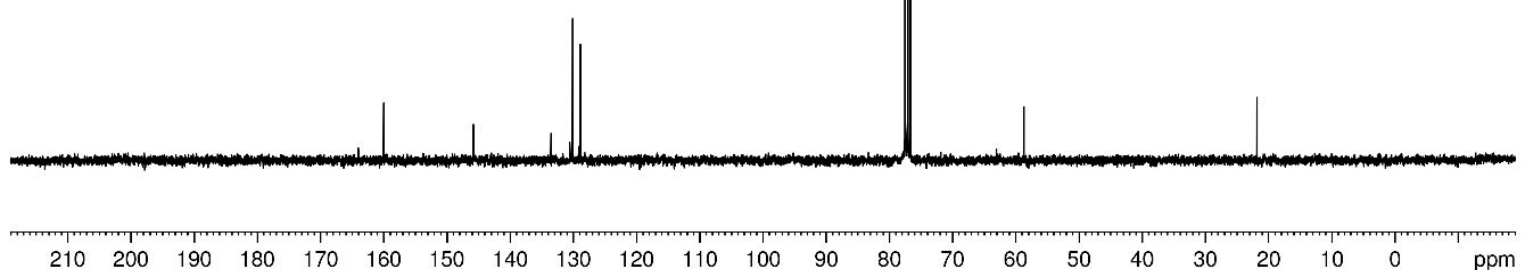


\section{Cartesian Coordinates and Energies of Calculated Structures}

Table S3. Single point energies of all structures calculated at the $\operatorname{IEFPCM}_{(\mathrm{THF})} \mathrm{B} 3 \mathrm{LYP} / 6-$ $311++\mathrm{G}(2 \mathrm{~d}, \mathrm{p})$ level of theory and thermal correction to Gibbs free energy, and Gibbs free energy calculated at the B3LYP/6-31+G(d,p) level of theory. All energies are reported in Hartrees.

\begin{tabular}{|c|c|c|c|c|}
\hline Structure & $\begin{array}{c}\text { Single Point } \\
\text { Energies (E) } \\
\text { IEFPCM }_{(\mathrm{THF})} \text { B3LY } \\
\text { P/6-311++G(2d,p) }\end{array}$ & $\begin{array}{c}\text { Thermal } \\
\text { Corrections } \\
\text { to Gibbs } \\
\text { Free } \\
\text { Energies } \\
\text { (G) } \\
\text { B3LYP/6- } \\
\text { 31+G(d,p) }\end{array}$ & $\begin{array}{c}\text { Gibbs Free } \\
\text { Energies } \\
\text { (G) } \\
\text { B3LYP/6- } \\
\text { 31+G(d,p) }\end{array}$ & $\begin{array}{c}\text { Gibbs Free Energies } \\
\text { (G) } \\
\left.\text { IEFPCM }_{(\mathrm{THF})} \text { B3LYP/ } / 2 d, p\right) / / \\
\text { 6-311++G(2) } \\
\text { B3LYP/6-31+G(d,p) }\end{array}$ \\
\hline $\begin{array}{l}\text { Methanesul } \\
\text { fonic Acid }\end{array}$ & -664.47412018 & 0.031842 & -664.300432 & -664.4422782 \\
\hline $2 a$ & -232.51291329 & 0.081917 & -232.371082 & -232.4309963 \\
\hline $\mathrm{H}_{2} \mathrm{O}$ & -76.46574793 & 0.003646 & -76.430402 & -76.46210193 \\
\hline $\begin{array}{l}\text { tert-Butyl } \\
\text { isocyanide }\end{array}$ & -250.75004670 & 0.099435 & -250.591377 & -250.6506117 \\
\hline IM1 & -896.99270026 & 0.131425 & -896.664597 & -896.8612753 \\
\hline TS1 & -896.97736282 & 0.132483 & -896.643884 & -896.8448798 \\
\hline IM2 & -897.00670311 & 0.140055 & -896.674249 & -896.8666481 \\
\hline IM3 & -1812.24661510 & 0.304729 & -1811.559677 & -1811.941886 \\
\hline TS2 & -1812.23665746 & 0.308097 & -1811.541688 & -1811.92856 \\
\hline IM4 & -1812.26270362 & 0.311387 & -1811.564006 & -1811.951317 \\
\hline $\mathbf{T S 3}_{\mathrm{C}}$ & -1812.24347080 & 0.313303 & -1811.547198 & -1811.930168 \\
\hline IM5 & -1812.24419506 & 0.312872 & -1811.549033 & -1811.931323 \\
\hline IM6 & -1888.73772805 & 0.332949 & -1887.992350 & -1888.404779 \\
\hline $\mathbf{T S 3}_{\mathrm{H}}$ & -1888.72836949 & 0.334561 & -1887.983974 & -1888.393808 \\
\hline IM7 & -1888.74541873 & 0.334954 & -1888.002003 & -1888.410465 \\
\hline 3' & -559.80513997 & 0.232907 & -559.431614 & -559.572233 \\
\hline
\end{tabular}

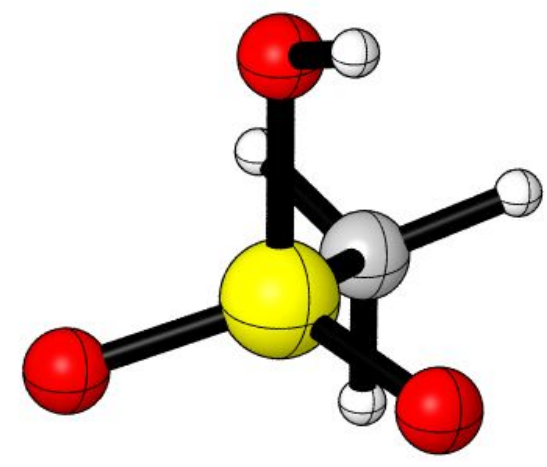


Figure S13. Optimized Structure of Methanesulfonic Acid.

Zero-point correction $=$

Thermal correction to Energy=

Thermal correction to Enthalpy=

Thermal correction to Gibbs Free Energy=

Sum of electronic and zero-point Energies $=$

Sum of electronic and thermal Energies=

Sum of electronic and thermal Enthalpies $=$

Sum of electronic and thermal Free Energies=
0.061232 (Hartree/Particle)

0.067282

0.068226

0.031842

$-664.271041$

$-664.264992$

$-664.264048$

$-664.300432$

Number of Imaginary Frequencies $=0$

E (Single Point Energy) $\left[\operatorname{IEFPCM}_{(\mathrm{THF})} \mathrm{B} 3 \mathrm{LYP} / 6-311++\mathrm{G}(2 \mathrm{~d}, \mathrm{p})\right]=-664.47412018$

$\begin{array}{lrrr}01 & & & \\ \mathrm{~S} & -0.09054600 & -0.15134000 & 0.04499100 \\ \mathrm{O} & -0.64932200 & -0.15187900 & 1.39939800 \\ \mathrm{O} & -0.23979900 & -1.30651800 & -0.83015500 \\ \mathrm{C} & 1.63773500 & 0.33319700 & 0.08210000 \\ \mathrm{H} & 2.18437900 & -0.49818400 & 0.53038400 \\ \mathrm{H} & 1.73432300 & 1.23587700 & 0.68496100 \\ \mathrm{H} & 1.96576800 & 0.50001400 & -0.94430000 \\ \mathrm{O} & -0.70881900 & 1.17218000 & -0.72781300 \\ \mathrm{H} & -1.47863400 & 1.47428900 & -0.21494200\end{array}$

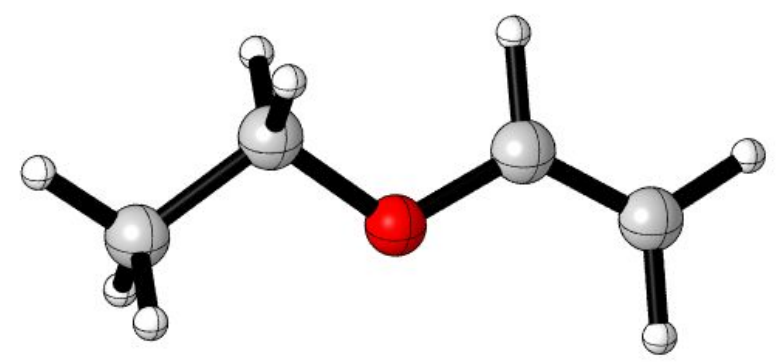

Figure S14. Optimized Structure of 2 a.

Zero-point correction=

Thermal correction to Energy=

Thermal correction to Enthalpy=

Thermal correction to Gibbs Free Energy=

Sum of electronic and zero-point Energies $=$

Sum of electronic and thermal Energies=
0.112572 (Hartree/Particle)

0.119106

0.120050

0.081917

$-232.340427$

$-232.333893$ 
Sum of electronic and thermal Enthalpies $=\quad-232.332949$

Sum of electronic and thermal Free Energies $=\quad-232.371082$

Number of Imaginary Frequencies $=0$

E (Single Point Energy) $\left[\operatorname{IEFPCM}_{(\mathrm{THF})} \mathrm{B} 3 \mathrm{LYP} / 6-311++\mathrm{G}(2 \mathrm{~d}, \mathrm{p})\right]=-232.51291329$

$\begin{array}{lrrr}01 & & & \\ \mathrm{C} & -2.30423500 & -0.34998200 & -0.02473900 \\ \mathrm{H} & -2.31243900 & -1.08777000 & 0.78302600 \\ \mathrm{H} & -2.32461800 & -0.88343600 & -0.97973200 \\ \mathrm{H} & -3.21049600 & 0.25973900 & 0.05292200 \\ \mathrm{C} & -1.07457300 & 0.53443800 & 0.06950000 \\ \mathrm{H} & -1.06272800 & 1.27582400 & -0.74256800 \\ \mathrm{H} & -1.05317800 & 1.07461000 & 1.02671000 \\ \mathrm{O} & 0.08713200 & -0.29929500 & -0.03162400 \\ \mathrm{C} & 1.28543900 & 0.34913600 & -0.04813000 \\ \mathrm{H} & 1.22775700 & 1.43385800 & -0.14462600 \\ \mathrm{C} & 2.45319900 & -0.29515500 & 0.02799300 \\ \mathrm{H} & 3.37892700 & 0.26532300 & -0.01566400 \\ \mathrm{H} & 2.50074900 & -1.37441100 & 0.12518300\end{array}$

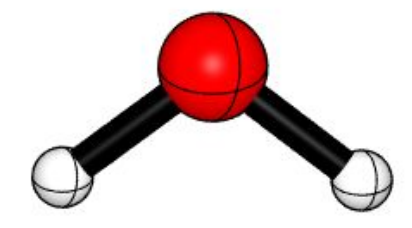

Figure S15. Optimized Structure of $\mathrm{H}_{2} \mathrm{O}$.

Zero-point correction $=$

Thermal correction to Energy=

Thermal correction to Enthalpy=

Thermal correction to Gibbs Free Energy=

Sum of electronic and zero-point Energies=

Sum of electronic and thermal Energies=

Sum of electronic and thermal Enthalpies=

Sum of electronic and thermal Free Energies=
0.021295 (Hartree/Particle)

0.024131

0.025075

0.003646

$-76.412753$

$-76.409918$

$-76.408973$

$-76.430402$

Number of Imaginary Frequencies $=0$

E (Single Point Energy) $\left[\operatorname{IEFPCM}_{(\mathrm{THF})} \mathrm{B} 3 \mathrm{LYP} / 6-311++\mathrm{G}(2 \mathrm{~d}, \mathrm{p})\right]=-76.46574793$
01
$\begin{array}{llll}\mathrm{O} & 0.00000000 & 0.00000000 & 0.11663600 \\ \mathrm{H} & 0.00000000 & 0.76884300 & -0.46654500\end{array}$
$\begin{array}{llll}\mathrm{H} & 0.00000000 & 0.76884300 & -0.46654500\end{array}$
$\mathrm{H} \quad 0.00000000 \quad-0.76884300 \quad-0.46654500$ 


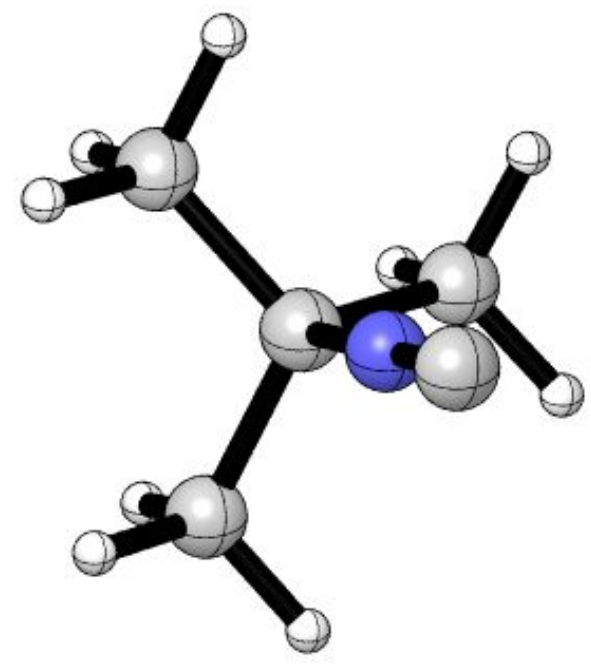

Figure S16. Optimized Structure of tert-Butyl isocyanide.

Zero-point correction=

Thermal correction to Energy=

Thermal correction to Enthalpy=

Thermal correction to Gibbs Free Energy=

Sum of electronic and zero-point Energies=

Sum of electronic and thermal Energies $=$

Sum of electronic and thermal Enthalpies=

Sum of electronic and thermal Free Energies=
0.129693 (Hartree/Particle)

0.137064

0.138008

0.099435

$-250.561119$

$-250.553748$

$-250.552804$

$-250.591377$

Number of Imaginary Frequencies $=0$

E (Single Point Energy) $\left[\operatorname{IEFPCM}_{(\mathrm{THF})} \mathrm{B} 3 \mathrm{LYP} / 6-311++\mathrm{G}(2 \mathrm{~d}, \mathrm{p})\right]=-250.75004670$

01

$\begin{array}{lrrr}\mathrm{C} & 2.36413400 & 0.00017700 & 0.00009900 \\ \mathrm{~N} & 1.18842400 & 0.00031300 & 0.00008000 \\ \mathrm{C} & -0.25745900 & -0.00000200 & -0.00000400 \\ \mathrm{C} & -0.73509500 & -0.96686900 & -1.09977700 \\ \mathrm{H} & -0.37162300 & -1.98055400 & -0.90875300 \\ \mathrm{H} & -0.37183800 & -0.64743100 & -2.08067600 \\ \mathrm{H} & -1.82914400 & -0.98666400 & -1.12211800 \\ \mathrm{C} & -0.73572500 & 1.43567900 & -0.28740300 \\ \mathrm{H} & -0.37257600 & 1.77725600 & -1.26085000 \\ \mathrm{H} & -1.82978400 & 1.46448100 & -0.29318900 \\ \mathrm{H} & -0.37264300 & 2.12557300 & 0.47964900 \\ \mathrm{C} & -0.73543500 & -0.46913800 & 1.38705300 \\ \mathrm{H} & -1.82949100 & -0.47892600 & 1.41501800 \\ \mathrm{H} & -0.37195100 & -1.47823400 & 1.60098500\end{array}$




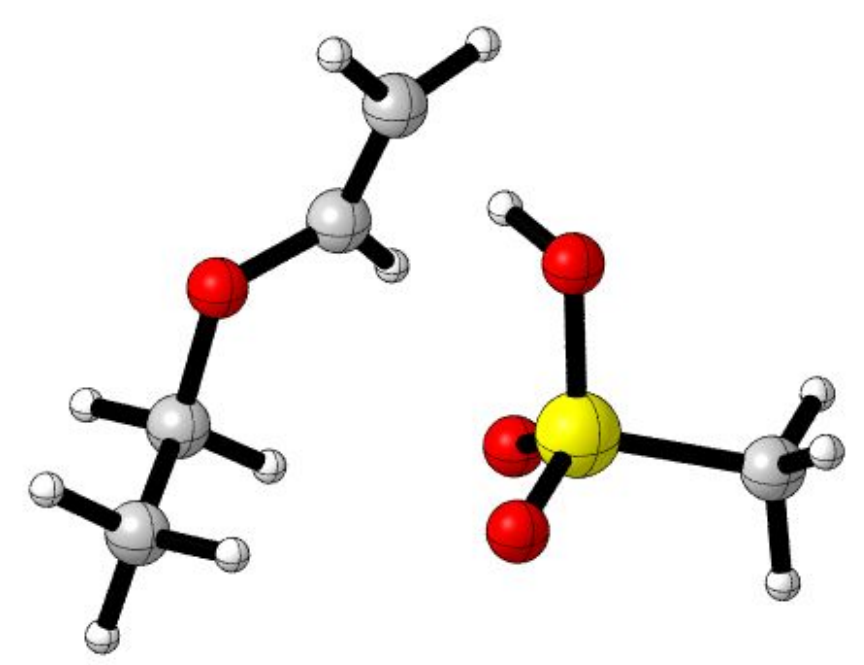

Figure S17. Optimized Structure of IM1.

Zero-point correction $=$

Thermal correction to Energy=

Thermal correction to Enthalpy=

Thermal correction to Gibbs Free Energy=

Sum of electronic and zero-point Energies=

Sum of electronic and thermal Energies=

Sum of electronic and thermal Enthalpies=

Sum of electronic and thermal Free Energies=
0.175567 (Hartree/Particle)

0.189424

0.190369

0.131425

$-896.620455$

$-896.606597$

$-896.605653$

$-896.664597$

Number of Imaginary Frequencies $=0$

E (Single Point Energy) [IEFPCM $($ THF) B3LYP/6-311++G(2d,p)] = -896.99270026

01

C $\quad 3.02881900 \quad-1.89961400 \quad-0.35521400$

$\mathrm{H} \quad 3.94041800-1.59862000-0.88038500$

$\mathrm{H} \quad 3.24821400 \quad-2.79491200 \quad 0.23580500$

$\mathrm{H} \quad 2.26461900 \quad-2.15276600-1.09542800$

$\begin{array}{llll}\mathrm{C} & 2.53840000 & -0.79395300 & 0.55870800\end{array}$

$\mathrm{H} \quad 1.61214500 \quad-1.07909200 \quad 1.06826300$

$\mathrm{H} \quad 3.29760900 \quad-0.53097100 \quad 1.30768800$

O $\quad 2.27581900 \quad 0.37194100 \quad-0.25863300$

$\begin{array}{llll}\mathrm{C} & 1.75678100 & 1.44176100 & 0.37224200\end{array}$

$\begin{array}{llll}\mathrm{H} & 1.68352500 & 1.36486000 & 1.45747600\end{array}$

C $\quad \begin{array}{llll}1.33171300 & 2.53410200 & -0.28788400\end{array}$

$\mathrm{H} \quad 1.43364600 \quad 2.61776100 \quad-1.36528800$ 


$\begin{array}{lrrr}\mathrm{H} & 0.97816000 & 3.39198400 & 0.27203200 \\ \mathrm{~S} & -1.64409600 & -0.31504200 & -0.01925400 \\ \mathrm{O} & -1.48828000 & 1.23964700 & -0.47813900 \\ \mathrm{O} & -0.87179700 & -0.51941800 & 1.21423400 \\ \mathrm{C} & -3.39907800 & -0.28026800 & 0.35329900 \\ \mathrm{H} & -3.93363100 & 0.01586900 & -0.54969200 \\ \mathrm{H} & -3.56903400 & 0.42387400 & 1.16745800 \\ \mathrm{H} & -3.67283300 & -1.29455200 & 0.64806500 \\ \mathrm{O} & -1.41177500 & -1.20162400 & -1.15813400 \\ \mathrm{H} & -0.54885600 & 1.54070000 & -0.35944900\end{array}$

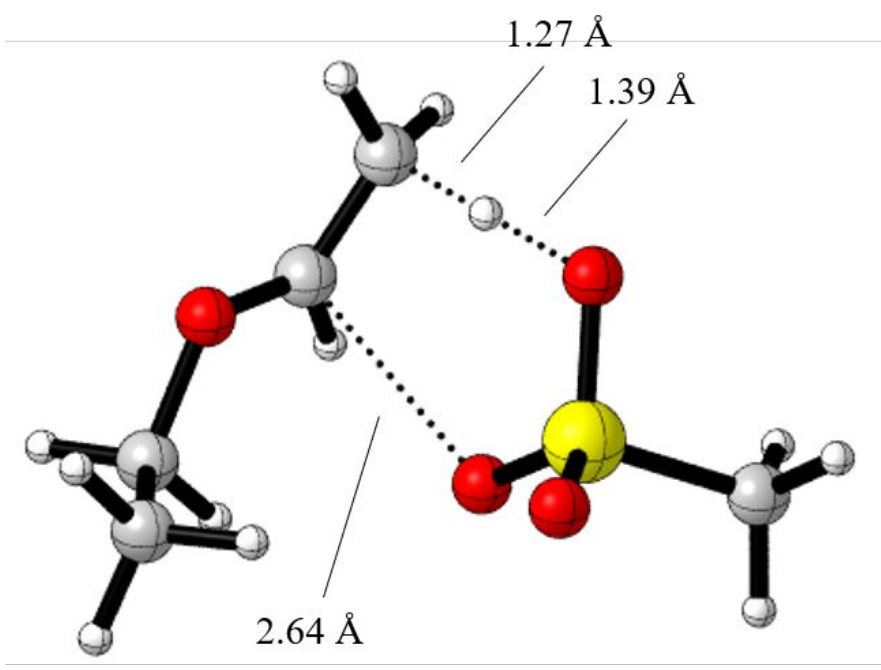

Figure S18. Optimized Structure of TS1.

Zero-point correction=

Thermal correction to Energy=

Thermal correction to Enthalpy=

Thermal correction to Gibbs Free Energy=

Sum of electronic and zero-point Energies=

Sum of electronic and thermal Energies $=$

Sum of electronic and thermal Enthalpies=

Sum of electronic and thermal Free Energies=
0.172675 (Hartree/Particle)

0.185020

0.185964

0.132483

$-896.603692$

$-896.591347$

$-896.590403$

$-896.643884$

Number of Imaginary Frequencies $=1$

E (Single Point Energy) [IEFPCM $($ THF) B3LYP/6-311++G(2d,p)] = -896.97736282

01

C $\quad 2.40000400 \quad-1.91165700 \quad-0.36653900$

$\mathrm{H} \quad 2.94698800-1.82980200-1.31016200$

$\mathrm{H} \quad 2.73234900 \quad-2.82100100 \quad 0.14582200$ 


$\begin{array}{lrrr}\mathrm{H} & 1.33034600 & -1.99813800 & -0.57192200 \\ \mathrm{C} & 2.67705100 & -0.72058600 & 0.52661900 \\ \mathrm{H} & 2.10329900 & -0.76877500 & 1.45430200 \\ \mathrm{H} & 3.74261900 & -0.59278900 & 0.73669000 \\ \mathrm{O} & 2.27089300 & 0.49786300 & -0.19433400 \\ \mathrm{C} & 1.52423000 & 1.37314200 & 0.39691800 \\ \mathrm{H} & 1.35667900 & 1.24940500 & 1.46541800 \\ \mathrm{C} & 0.86672300 & 2.37556700 & -0.33207000 \\ \mathrm{H} & 1.25055300 & 2.57804500 & -1.33123900 \\ \mathrm{H} & 0.57079100 & 3.24793600 & 0.24839900 \\ \mathrm{~S} & -1.47763500 & -0.27225700 & -0.06733900 \\ \mathrm{O} & -1.38687100 & 1.05364900 & -0.84525900 \\ \mathrm{O} & -0.42890500 & -0.29499500 & 1.00234300 \\ \mathrm{C} & -3.07516400 & -0.18162800 & 0.76125800 \\ \mathrm{H} & -3.84764000 & -0.10655100 & -0.00531800 \\ \mathrm{H} & -3.08446900 & 0.69499900 & 1.40988600 \\ \mathrm{H} & -3.19870600 & -1.09710700 & 1.34212300 \\ \mathrm{O} & -1.50442900 & -1.45177400 & -0.94752500 \\ \mathrm{H} & -0.22321600 & 1.75290200 & -0.54548800\end{array}$

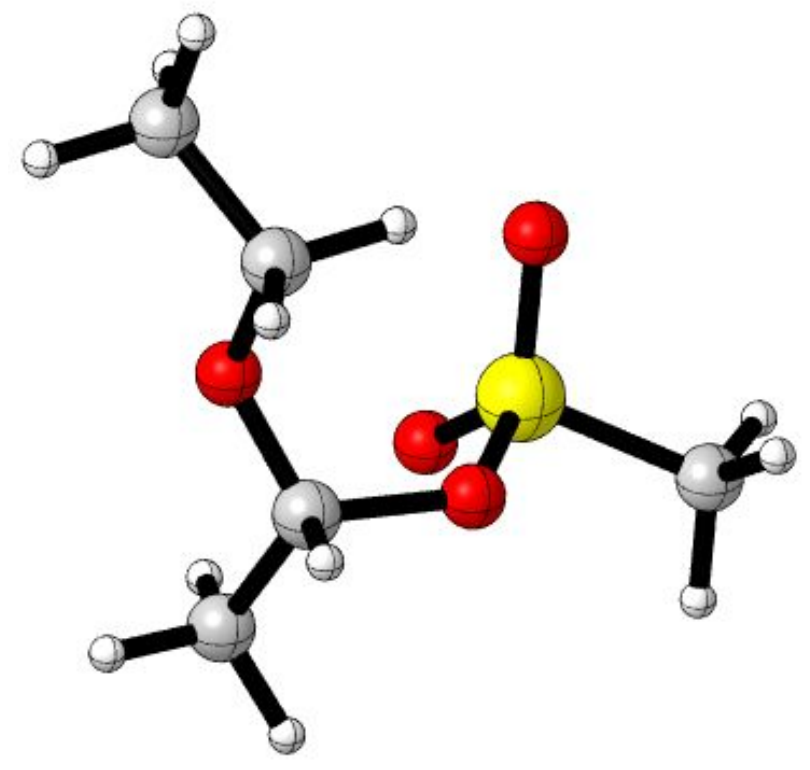

Figure S19. Optimized Structure of IM2.

Zero-point correction=

Thermal correction to Energy=

Thermal correction to Enthalpy=

Thermal correction to Gibbs Free Energy=

Sum of electronic and zero-point Energies=

Sum of electronic and thermal Energies=
0.179091 (Hartree/Particle)

0.191395

0.192339

0.140055

$-896.635214$

$-896.622910$ 


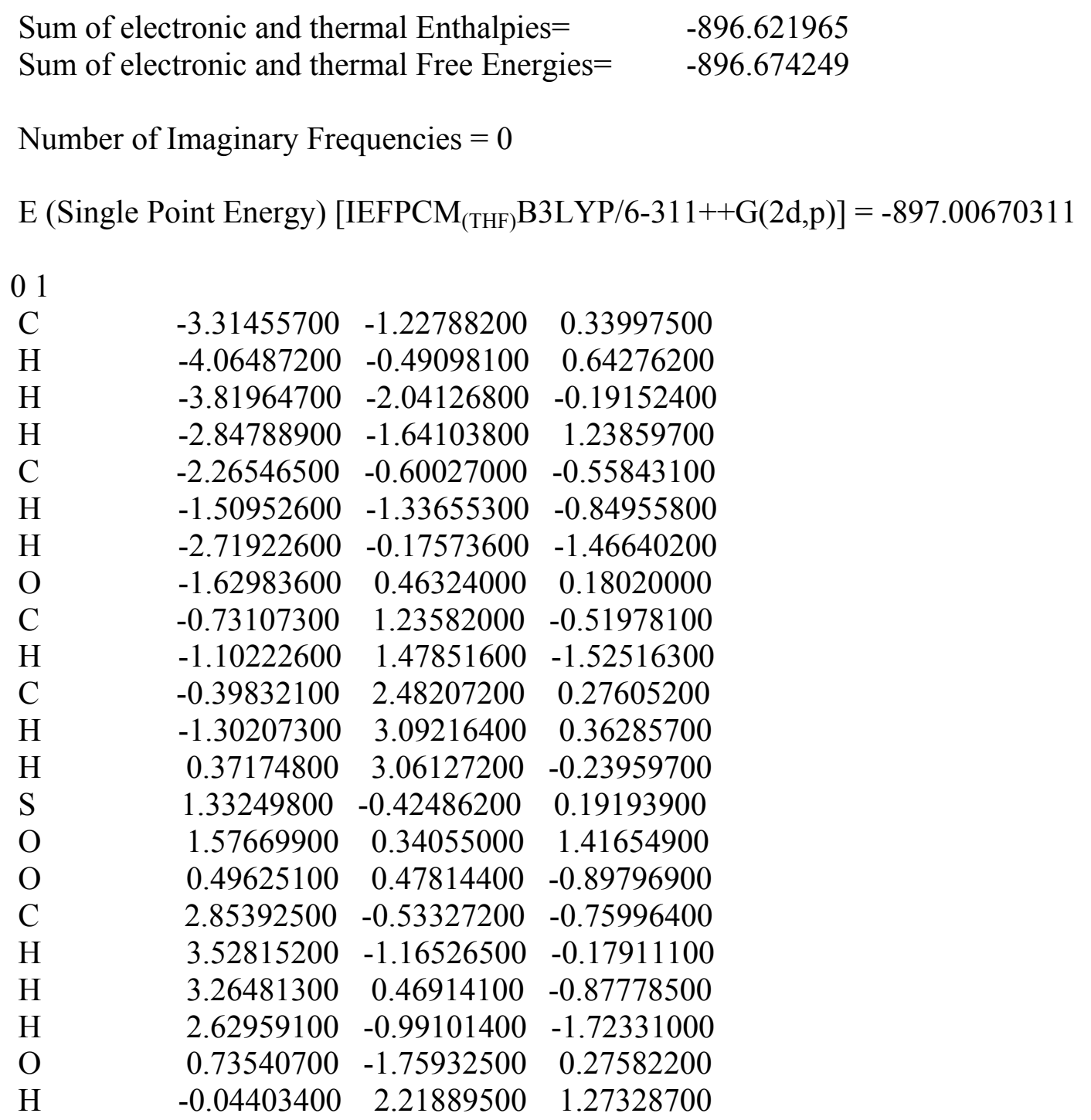




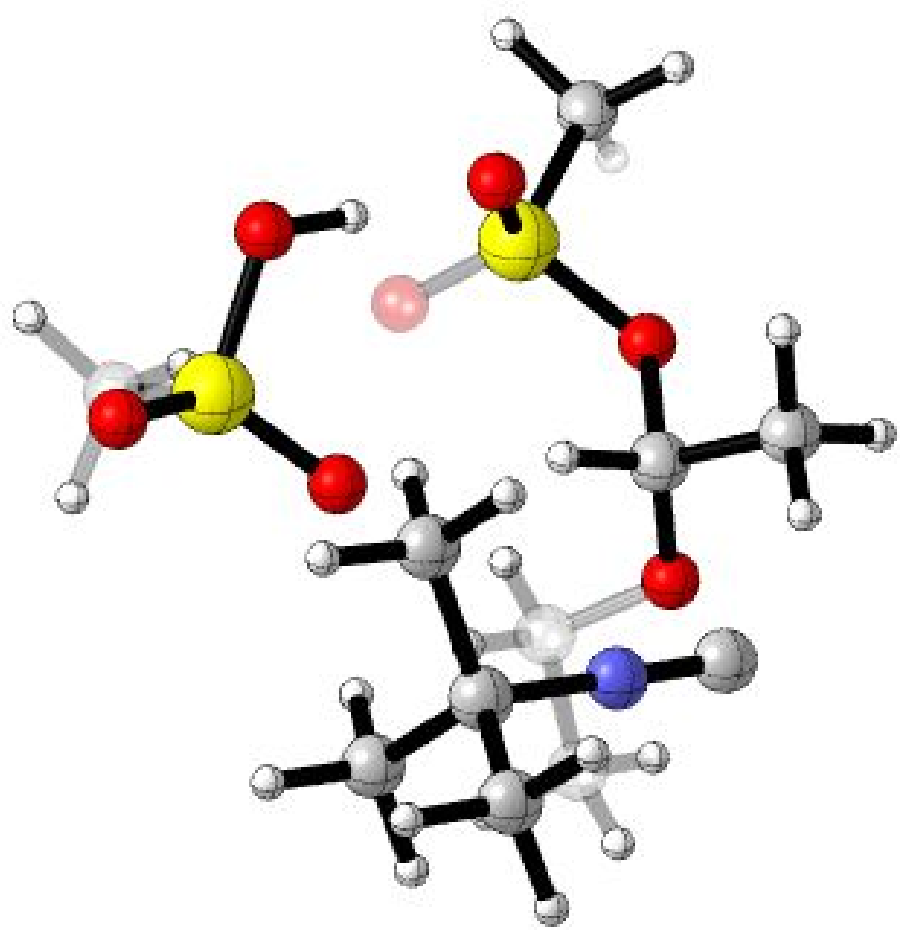

Figure S20. Optimized Structure of IM3.

Zero-point correction $=$

Thermal correction to Energy=

Thermal correction to Enthalpy=

Thermal correction to Gibbs Free Energy=

Sum of electronic and zero-point Energies $=$

Sum of electronic and thermal Energies=

Sum of electronic and thermal Enthalpies=

Sum of electronic and thermal Free Energies=
0.372496 (Hartree/Particle)

0.401981

0.402925

0.304729

$-1811.491910$

$-1811.462425$

$-1811.461481$

$-1811.559677$

Number of Imaginary Frequencies $=0$

E (Single Point Energy) $\left[\operatorname{IEFPCM}_{(\mathrm{THF})} \mathrm{B} 3 \mathrm{LYP} / 6-311++\mathrm{G}(2 \mathrm{~d}, \mathrm{p})\right]=-1812.24661510$

01

C $\quad-1.32049900 \quad-3.02838300 \quad 3.07161600$

$\mathrm{H} \quad-0.36457200 \quad-3.55715900 \quad 3.01240600$

$\mathrm{H} \quad-1.39671200 \quad-2.55842300 \quad 4.05779300$

$\mathrm{H} \quad-2.12795900 \quad-3.76055800 \quad 2.97531200$

C $\quad-1.42009400 \quad-1.96963300 \quad 1.98985100$

$\mathrm{H} \quad-2.37659000 \quad-1.43631700 \quad 2.03893600$

$\mathrm{H} \quad-0.61037800 \quad-1.23410300 \quad 2.06860400$

$\begin{array}{llll}\mathrm{O} & -1.32009600 & -2.63398900 & 0.71028500\end{array}$

C $\quad-1.25709600 \quad-1.82561000-0.38312900$ 


$\begin{array}{lccc}\mathrm{H} & -0.68508300 & -0.90997000 & -0.20585400 \\ \mathrm{C} & -0.81850000 & -2.59620300 & -1.60110000 \\ \mathrm{H} & 0.21472200 & -2.92636700 & -1.46048700 \\ \mathrm{H} & -0.86622000 & -1.95782000 & -2.48620500 \\ \mathrm{~S} & -3.07597500 & 0.19329600 & -0.74108100 \\ \mathrm{O} & -2.71050200 & -1.35852600 & -0.68567100 \\ \mathrm{O} & -2.14233000 & 0.89845200 & -1.65294300 \\ \mathrm{C} & -4.67455300 & 0.06842400 & -1.54192300 \\ \mathrm{H} & -5.32289000 & -0.54110500 & -0.91217600 \\ \mathrm{H} & -4.53347500 & -0.38153700 & -2.52437300 \\ \mathrm{H} & -5.05750800 & 1.08675000 & -1.62684000 \\ \mathrm{O} & -3.23834600 & 0.76061000 & 0.60376600 \\ \mathrm{H} & -1.46188800 & -3.46895300 & -1.74172000 \\ \mathrm{~S} & 0.34018100 & 2.43376400 & 0.55019900 \\ \mathrm{O} & 0.45053900 & 0.96996500 & 0.66477000 \\ \mathrm{O} & -0.36865400 & 2.79677900 & -0.85508600 \\ \mathrm{C} & -0.86344900 & 3.01010300 & 1.75412600 \\ \mathrm{H} & -0.43414100 & 2.83155700 & 2.74168100 \\ \mathrm{H} & -1.78414400 & 2.44003600 & 1.61797400 \\ \mathrm{H} & -1.01892900 & 4.07671200 & 1.58896300 \\ \mathrm{O} & 1.53944700 & 3.26063800 & 0.63419400 \\ \mathrm{H} & -0.99404600 & 2.07492800 & -1.13507000 \\ \mathrm{C} & 2.93802600 & -2.48655700 & -1.15234000 \\ \mathrm{~N} & 3.61360300 & -1.59379800 & -0.79798800 \\ \mathrm{C} & 4.43179000 & -0.48279200 & -0.35687000 \\ \mathrm{C} & 4.18018700 & 0.70138900 & -1.30857200 \\ \mathrm{H} & 3.13079500 & 1.00380400 & -1.28066000 \\ \mathrm{H} & 4.44587700 & 0.43544600 & -2.33603000 \\ \mathrm{H} & 4.79012900 & 1.55499200 & -0.99814500 \\ \mathrm{C} & 5.90451100 & -0.92982000 & -0.41140300 \\ \mathrm{H} & 6.18934000 & -1.20792100 & -1.43036000 \\ \mathrm{H} & 6.54741000 & -0.10777800 & -0.08191100 \\ \mathrm{H} & 6.07433100 & -1.78915100 & 0.24390900 \\ \mathrm{C} & 4.01417500 & -0.12667900 & 1.08212500 \\ \mathrm{H} & 4.61832800 & 0.71374600 & 1.43767500 \\ \mathrm{H} & 2.96153400 & 0.16446300 & 1.11707900 \\ \mathrm{H} & 4.17209900 & -0.97849800 & 1.75071700\end{array}$




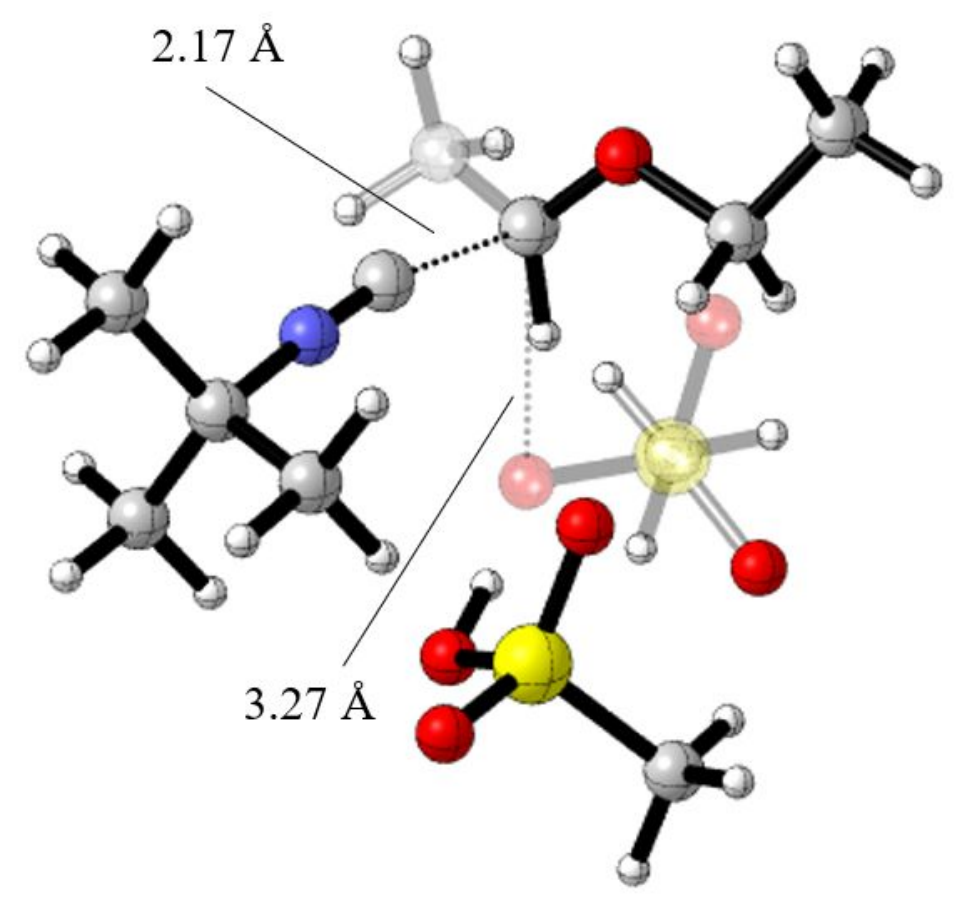

Figure S21. Optimized Structure of TS2.

Zero-point correction=

Thermal correction to Energy=

Thermal correction to Enthalpy=

Thermal correction to Gibbs Free Energy=

Sum of electronic and zero-point Energies=

Sum of electronic and thermal Energies $=$

Sum of electronic and thermal Enthalpies=

Sum of electronic and thermal Free Energies=
0.370942 (Hartree/Particle)

0.399519

0.400464

0.308097

$-1811.478843$

$-1811.450265$

$-1811.449321$

$-1811.541688$

Number of Imaginary Frequencies $=1$

E (Single Point Energy) [IEFPCM $(\mathrm{THF})$ B3LYP/6-311++G(2d,p)] = -1812.23665746

01

$\begin{array}{lrrr}\mathrm{C} & 0.82523200 & 3.47812600 & 2.76493100 \\ \mathrm{H} & -0.06733200 & 4.11115800 & 2.77675700 \\ \mathrm{H} & 0.98423100 & 3.08947400 & 3.77590800 \\ \mathrm{H} & 1.69019400 & 4.09074400 & 2.49534100 \\ \mathrm{C} & 0.66764700 & 2.31416300 & 1.81248200 \\ \mathrm{H} & 1.56035800 & 1.68872400 & 1.75127500 \\ \mathrm{H} & -0.20243400 & 1.69198600 & 2.03643700 \\ \mathrm{O} & 0.46309200 & 2.87392300 & 0.46146300 \\ \mathrm{C} & 0.34515000 & 2.03911100 & -0.51675900\end{array}$




$\begin{array}{lrrr}\mathrm{H} & 0.55908800 & 0.99082300 & -0.33395600 \\ \mathrm{C} & 0.49816200 & 2.59963100 & -1.88522200 \\ \mathrm{H} & 0.10987400 & 1.90923200 & -2.63428200 \\ \mathrm{H} & 1.58031100 & 2.69761100 & -2.03600200 \\ \mathrm{~S} & 3.15035500 & -0.25409000 & -0.56408700 \\ \mathrm{O} & 3.04683400 & 1.17958000 & -0.16665500 \\ \mathrm{O} & 1.96352900 & -0.64506400 & -1.44631900 \\ \mathrm{C} & 4.57983100 & -0.38435700 & -1.65575300 \\ \mathrm{H} & 5.46087900 & -0.08901200 & -1.08403800 \\ \mathrm{H} & 4.42939000 & 0.28171600 & -2.50620600 \\ \mathrm{H} & 4.66282000 & -1.42100600 & -1.98461800 \\ \mathrm{O} & 3.36284400 & -1.21386000 & 0.54605400 \\ \mathrm{H} & 0.03025700 & 3.58175600 & -1.97347300 \\ \mathrm{~S} & -0.31596400 & -2.12805200 & 0.71342600 \\ \mathrm{O} & -0.24360300 & -0.70484400 & 1.10394000 \\ \mathrm{O} & 0.21844500 & -2.30706000 & -0.76855900 \\ \mathrm{C} & 0.86971200 & -3.04188700 & 1.70288000 \\ \mathrm{H} & 0.55037100 & -2.96242700 & 2.74349900 \\ \mathrm{H} & 1.84877600 & -2.58152800 & 1.54671600 \\ \mathrm{H} & 0.85258100 & -4.08029200 & 1.36973200 \\ \mathrm{O} & -1.62803900 & -2.78566200 & 0.78485600 \\ \mathrm{H} & 0.98520400 & -1.62896500 & -1.01070500 \\ \mathrm{C} & -1.74574800 & 1.45017900 & -0.55961000 \\ \mathrm{~N} & -2.77428800 & 0.90488200 & -0.56122300 \\ \mathrm{C} & -4.02966700 & 0.16583800 & -0.53706000 \\ \mathrm{C} & -3.88067900 & -1.03170300 & -1.49369100 \\ \mathrm{H} & -3.08840800 & -1.70312300 & -1.15468100 \\ \mathrm{H} & -3.66216200 & -0.69477500 & -2.51131300 \\ \mathrm{H} & -4.82309300 & -1.58745800 & -1.50775800 \\ \mathrm{C} & -5.13840500 & 1.12635200 & -1.00210700 \\ \mathrm{H} & -4.94996300 & 1.48124600 & -2.01960900 \\ \mathrm{H} & -6.09539500 & 0.59699400 & -0.99240600 \\ \mathrm{H} & -5.21450600 & 1.99083700 & -0.33602700 \\ \mathrm{C} & -4.25997400 & -0.30867600 & 0.90936200 \\ \mathrm{H} & -5.20570800 & -0.85736200 & 0.95156800 \\ \mathrm{H} & -3.45571400 & -0.97555900 & 1.22805200 \\ & -4.32225400 & 0.54255600 & 1.59396300\end{array}$




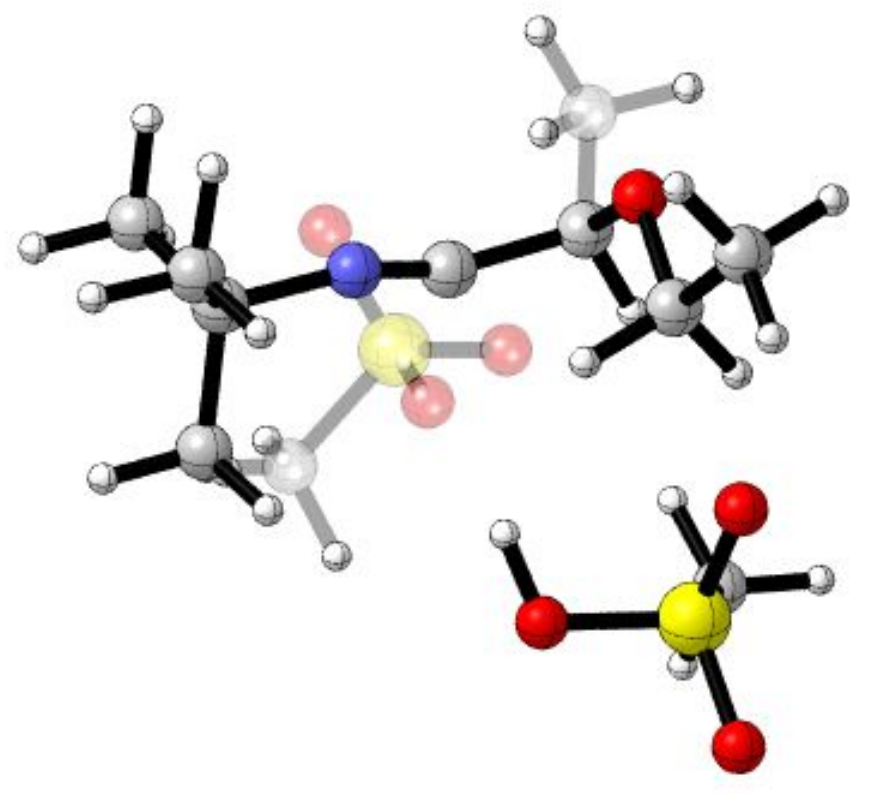

Figure S22. Optimized Structure of IM4.

Zero-point correction $=$

Thermal correction to Energy=

Thermal correction to Enthalpy=

Thermal correction to Gibbs Free Energy=

Sum of electronic and zero-point Energies=

Sum of electronic and thermal Energies $=$

Sum of electronic and thermal Enthalpies=

Sum of electronic and thermal Free Energies=
0.373508 (Hartree/Particle)

0.401977

0.402921

0.311387

$-1811.501886$

$-1811.473416$

$-1811.472472$

$-1811.564006$

Number of Imaginary Frequencies $=0$

E (Single Point Energy) [IEFPCM $($ THF) B3LYP/6-311++G(2d,p)] = -1812.26270362

01

$\begin{array}{lrrr}\mathrm{C} & -3.38516500 & 3.33335200 & -1.24866500 \\ \mathrm{H} & -4.19411800 & 3.00463900 & -0.58907100 \\ \mathrm{H} & -3.07088600 & 4.33375100 & -0.93371600 \\ \mathrm{H} & -3.77452900 & 3.39712300 & -2.26931800 \\ \mathrm{C} & -2.20680600 & 2.38131800 & -1.17440000 \\ \mathrm{H} & -1.39129800 & 2.70611900 & -1.83540800 \\ \mathrm{H} & -1.80428600 & 2.30965000 & -0.15854200 \\ \mathrm{O} & -2.66672500 & 1.08370300 & -1.61751200 \\ \mathrm{C} & -1.69146800 & 0.06779600 & -1.65335100 \\ \mathrm{H} & -0.67725800 & 0.43964700 & -1.85854700 \\ \mathrm{C} & -2.09147300 & -0.98412700 & -2.69125200\end{array}$




$\begin{array}{lccc}\mathrm{H} & -1.38332200 & -1.81380500 & -2.67027800 \\ \mathrm{H} & -2.06947700 & -0.50338300 & -3.67200200 \\ \mathrm{~S} & 1.68362400 & -1.51813600 & -1.14944500 \\ \mathrm{O} & 0.38917700 & -2.26450700 & -1.09814600 \\ \mathrm{O} & 2.26756300 & -1.31111900 & 0.23163600 \\ \mathrm{C} & 2.86289600 & -2.59426000 & -1.98694500 \\ \mathrm{H} & 2.48702000 & -2.78925700 & -2.99231400 \\ \mathrm{H} & 2.94416500 & -3.52021100 & -1.41632300 \\ \mathrm{H} & 3.82354700 & -2.07915000 & -2.02620300 \\ \mathrm{O} & 1.60657200 & -0.25341900 & -1.93737900 \\ \mathrm{H} & -3.10622300 & -1.34274500 & -2.49845000 \\ \mathrm{~S} & 1.60549900 & 2.08943300 & 1.06222800 \\ \mathrm{O} & 0.25345700 & 1.53707200 & 0.83198100 \\ \mathrm{O} & 2.60733400 & 0.90981100 & 1.43972300 \\ \mathrm{C} & 2.24408300 & 2.67194200 & -0.51425900 \\ \mathrm{H} & 1.61884300 & 3.51103400 & -0.82524100 \\ \mathrm{H} & 2.18692400 & 1.84456400 & -1.22560900 \\ \mathrm{H} & 3.27196100 & 2.99999300 & -0.35480300 \\ \mathrm{O} & 1.77060400 & 3.13765000 & 2.06791100 \\ \mathrm{H} & 2.43037200 & 0.04567300 & 0.90820600 \\ \mathrm{C} & -1.61475900 & -0.56504300 & -0.30149100 \\ \mathrm{~N} & -1.69802800 & -1.02153100 & 0.75120700 \\ \mathrm{C} & -1.61693300 & -1.71766800 & 2.03581200 \\ \mathrm{C} & -1.62948400 & -3.22226900 & 1.70859700 \\ \mathrm{H} & -0.80202300 & -3.47205200 & 1.04034100 \\ \mathrm{H} & -2.57279400 & -3.51670100 & 1.23869900 \\ \mathrm{H} & -1.51892100 & -3.77931200 & 2.64333400 \\ \mathrm{C} & -2.84147000 & -1.29349300 & 2.86275500 \\ \mathrm{H} & -3.77495900 & -1.55870200 & 2.35766400 \\ \mathrm{H} & -2.80887800 & -1.81438500 & 3.82353900 \\ \mathrm{H} & -2.83158900 & -0.21710500 & 3.05422000 \\ \mathrm{C} & -0.29729000 & -1.29366000 & 2.70220200 \\ \mathrm{H} & -0.22477800 & -1.80818300 & 3.66511500 \\ \mathrm{H} & 0.55857800 & -1.56893700 & 2.08185100 \\ \mathrm{H} & -0.27248100 & -0.21515000 & 2.87016700 \\ & & & \\ & & & \end{array}$




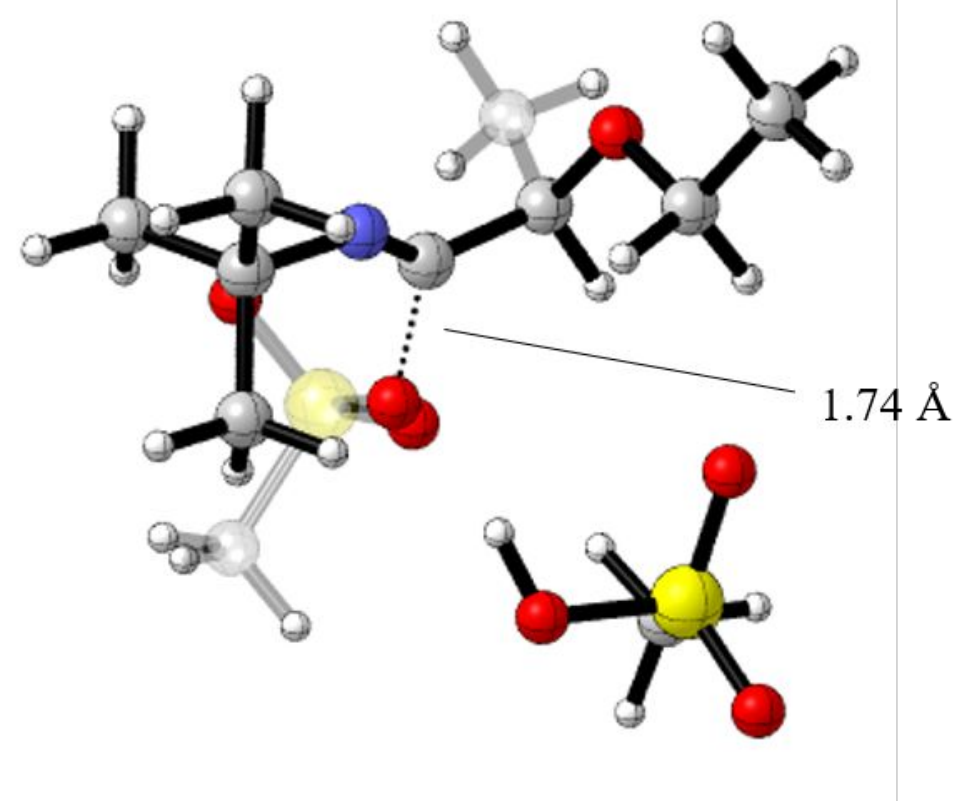

Figure S23. Optimized Structure of $\mathbf{T S 3} \mathbf{C}_{\mathbf{C}}$.

Zero-point correction=

Thermal correction to Energy=

Thermal correction to Enthalpy=

Thermal correction to Gibbs Free Energy=

Sum of electronic and zero-point Energies $=$

Sum of electronic and thermal Energies=

Sum of electronic and thermal Enthalpies=

Sum of electronic and thermal Free Energies=
0.373775 (Hartree/Particle)

0.401323

0.402267

0.313303

$-1811.486726$

$-1811.459178$

$-1811.458234$

$-1811.547198$

Number of Imaginary Frequencies $=1$

E (Single Point Energy) $\left[\operatorname{IEFPCM}_{(\mathrm{THF})} \mathrm{B} 3 \mathrm{LYP} / 6-311++\mathrm{G}(2 \mathrm{~d}, \mathrm{p})\right]=-1812.24347080$

01

$\begin{array}{lrrr}\mathrm{C} & 1.05536400 & 4.71251500 & 0.36886300 \\ \mathrm{H} & 2.14838200 & 4.75644800 & 0.40204900 \\ \mathrm{H} & 0.70846300 & 5.32022600 & -0.47352200 \\ \mathrm{H} & 0.66389100 & 5.14764700 & 1.29332100 \\ \mathrm{C} & 0.57674500 & 3.28081700 & 0.20468700 \\ \mathrm{H} & -0.51827700 & 3.22456600 & 0.17387800 \\ \mathrm{H} & 0.96570200 & 2.85098400 & -0.73024900 \\ \mathrm{O} & 1.06457200 & 2.52166300 & 1.32265700 \\ \mathrm{C} & 0.57628500 & 1.20323600 & 1.41603100 \\ \mathrm{H} & -0.49825300 & 1.16737700 & 1.20490600 \\ \mathrm{C} & 0.86780700 & 0.70353700 & 2.83347700 \\ \mathrm{H} & 0.53906100 & -0.32641200 & 2.97906500\end{array}$




$\begin{array}{lrrr}\mathrm{H} & 0.33429800 & 1.34899200 & 3.53535600 \\ \mathrm{~S} & -0.12058900 & -2.22121300 & 0.85411600 \\ \mathrm{O} & 1.12401800 & -2.55834500 & 1.55563900 \\ \mathrm{O} & 0.11530500 & -0.90893100 & -0.04550100 \\ \mathrm{C} & -0.43100600 & -3.45748400 & -0.41975500 \\ \mathrm{H} & -0.65969400 & -4.38699700 & 0.10490100 \\ \mathrm{H} & 0.46820900 & -3.56898000 & -1.02565400 \\ \mathrm{H} & -1.28027800 & -3.13433000 & -1.02289700 \\ \mathrm{O} & -1.35462400 & -2.05408300 & 1.63597600 \\ \mathrm{H} & 1.93996100 & 0.76834800 & 3.03716000 \\ \mathrm{~S} & -3.25777400 & 0.57532000 & -0.79505300 \\ \mathrm{O} & -2.51703400 & 1.55934700 & 0.00645200 \\ \mathrm{O} & -2.17626000 & -0.42976700 & -1.48355300 \\ \mathrm{C} & -4.14551500 & -0.52069000 & 0.31878400 \\ \mathrm{H} & -4.87548900 & 0.09469600 & 0.84775200 \\ \mathrm{H} & -3.42876700 & -0.96863700 & 1.00949400 \\ \mathrm{H} & -4.64697300 & -1.27824200 & -0.28450300 \\ \mathrm{O} & -4.15086300 & 1.00660800 & -1.86308800 \\ \mathrm{H} & -1.34903400 & -0.47143600 & -0.93696300 \\ \mathrm{C} & 1.26548700 & 0.32463700 & 0.37778400 \\ \mathrm{~N} & 2.31319400 & 0.36672600 & -0.21227900 \\ \mathrm{C} & 3.13192300 & -0.33255100 & -1.20688600 \\ \mathrm{C} & 3.81420300 & -1.51504100 & -0.49168700 \\ \mathrm{H} & 3.07952900 & -2.21230300 & -0.08277300 \\ \mathrm{H} & 4.43844100 & -1.15804500 & 0.33265600 \\ \mathrm{H} & 4.45505200 & -2.04329000 & -1.20474000 \\ \mathrm{C} & 4.18850300 & 0.67274300 & -1.69961400 \\ \mathrm{H} & 4.78627700 & 1.04457400 & -0.86281900 \\ \mathrm{H} & 4.85602200 & 0.18914700 & -2.41946400 \\ \mathrm{H} & 3.71423300 & 1.52818300 & -2.19027400 \\ \mathrm{C} & 2.26696700 & -0.81084200 & -2.38698900 \\ \mathrm{H} & 2.91184600 & -1.27601400 & -3.13908700 \\ \mathrm{H} & 1.51906200 & -1.53705300 & -2.06684300 \\ \mathrm{H} & 1.74829100 & 0.03055900 & -2.85663400 \\ & & & \\ & & & \\ \mathrm{H} & & & \end{array}$




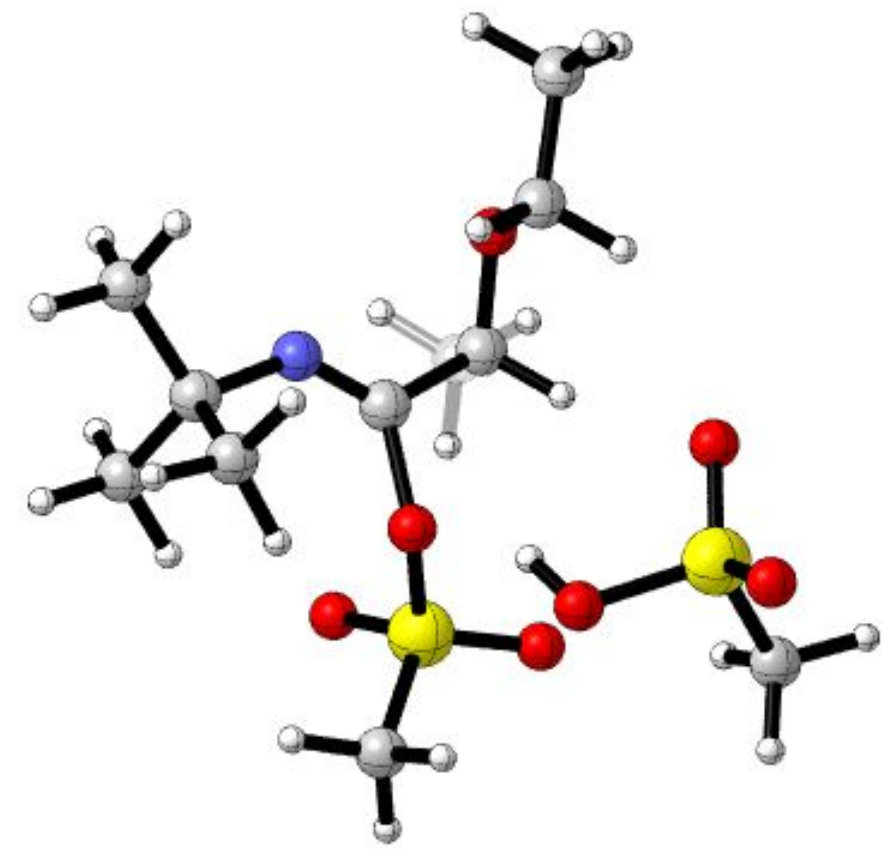

Figure S24. Optimized Structure of IM5.

Zero-point correction=

Thermal correction to Energy=

Thermal correction to Enthalpy=

Thermal correction to Gibbs Free Energy=

Sum of electronic and zero-point Energies=

Sum of electronic and thermal Energies=

Sum of electronic and thermal Enthalpies=

Sum of electronic and thermal Free Energies=
0.374439 (Hartree/Particle)

0.402389

0.403333

0.312872

$-1811.487466$

$-1811.459516$

$-1811.458572$

$-1811.549033$

Number of Imaginary Frequencies $=0$

E (Single Point Energy) [IEFPCM $(\mathrm{THF})$ B3LYP/6-311++G(2d,p)] = -1812.24419506

$\begin{array}{lrrr}01 & & & \\ \mathrm{C} & 1.03581800 & 4.60570400 & 0.47968700 \\ \mathrm{H} & 2.12288700 & 4.67608500 & 0.37476400 \\ \mathrm{H} & 0.57036900 & 5.20995600 & -0.30628200 \\ \mathrm{H} & 0.75504300 & 5.02519700 & 1.45069500 \\ \mathrm{C} & 0.57688600 & 3.16231800 & 0.36426900 \\ \mathrm{H} & -0.51278400 & 3.08130000 & 0.46759500 \\ \mathrm{H} & 0.85593500 & 2.75026300 & -0.61605300 \\ \mathrm{O} & 1.21742000 & 2.41255600 & 1.40413900 \\ \mathrm{C} & 0.79657700 & 1.07417000 & 1.54087200 \\ \mathrm{H} & -0.30135600 & 1.01814500 & 1.54180000\end{array}$




$\begin{array}{lrrr}\mathrm{C} & 1.35417700 & 0.57792400 & 2.87821800 \\ \mathrm{H} & 1.08480000 & -0.46009800 & 3.08033400 \\ \mathrm{H} & 0.95405200 & 1.20974600 & 3.67519800 \\ \mathrm{~S} & -0.06466300 & -2.15624500 & 0.96426800 \\ \mathrm{O} & 1.15365500 & -2.60292400 & 1.63569700 \\ \mathrm{O} & 0.30179100 & -0.86164800 & -0.00997600 \\ \mathrm{C} & -0.52019300 & -3.32218100 & -0.32624400 \\ \mathrm{H} & -0.83409000 & -4.23092000 & 0.19124600 \\ \mathrm{H} & 0.35573700 & -3.51201500 & -0.94609800 \\ \mathrm{H} & -1.34141200 & -2.89851700 & -0.90526100 \\ \mathrm{O} & -1.25464100 & -1.82149800 & 1.75029400 \\ \mathrm{H} & 2.44409200 & 0.66532500 & 2.87110900 \\ \mathrm{~S} & -3.28761500 & 0.57642900 & -0.78448800 \\ \mathrm{O} & -2.55401400 & 1.63870600 & -0.08936900 \\ \mathrm{O} & -2.17831100 & -0.47932800 & -1.37591000 \\ \mathrm{C} & -4.14569400 & -0.43346300 & 0.42919900 \\ \mathrm{H} & -4.89370900 & 0.20975800 & 0.89630500 \\ \mathrm{H} & -3.41873000 & -0.79104100 & 1.15971000 \\ \mathrm{H} & -4.62542600 & -1.25931300 & -0.09716400 \\ \mathrm{O} & -4.19269700 & 0.87529500 & -1.88569700 \\ \mathrm{H} & -1.32590000 & -0.35381600 & -0.90261400 \\ \mathrm{C} & 1.29007000 & 0.21172500 & 0.37016700 \\ \mathrm{~N} & 2.31845000 & 0.40409800 & -0.29020600 \\ \mathrm{C} & 2.91730300 & -0.30531200 & -1.43494200 \\ \mathrm{C} & 3.47947300 & -1.65603500 & -0.95027400 \\ \mathrm{H} & 2.69194800 & -2.31715900 & -0.58237700 \\ \mathrm{H} & 4.19520500 & -1.50512800 & -0.13670900 \\ \mathrm{H} & 3.99831900 & -2.15407700 & -1.77596200 \\ \mathrm{C} & 4.07580100 & 0.58495000 & -1.92412200 \\ \mathrm{H} & 4.78996000 & 0.76088600 & -1.11457200 \\ \mathrm{H} & 4.60030100 & 0.10812400 & -2.75869000 \\ \mathrm{H} & 3.70002600 & 1.55635900 & -2.25999300 \\ \mathrm{C} & 1.90742900 & -0.49935000 & -2.58378600 \\ \mathrm{H} & 2.42664600 & -0.90230900 & -3.45944700 \\ \mathrm{H} & 1.10493000 & -1.18755800 & -2.31342600 \\ \mathrm{H} & 1.45895400 & 0.45718700 & -2.87085100\end{array}$




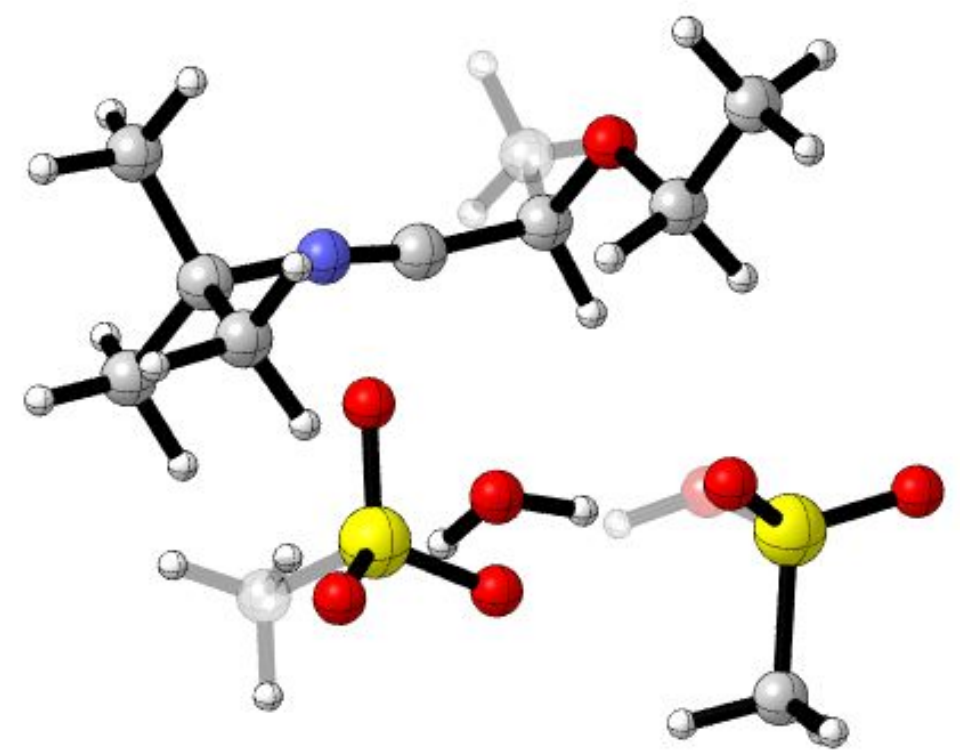

Figure S25. Optimized Structure of IM6.

Zero-point correction=

Thermal correction to Energy=

Thermal correction to Enthalpy=

Thermal correction to Gibbs Free Energy=

Sum of electronic and zero-point Energies=

Sum of electronic and thermal Energies=

Sum of electronic and thermal Enthalpies=

Sum of electronic and thermal Free Energies=
0.398868 (Hartree/Particle)

0.430112

0.431056

0.332949

$-1887.926431$

$-1887.895187$

$-1887.894243$

$-1887.992350$

Number of Imaginary Frequencies $=0$

E (Single Point Energy) $\left[\operatorname{IEFPCM}_{(\mathrm{THF})} \mathrm{B} 3 \mathrm{LYP} / 6-311++\mathrm{G}(2 \mathrm{~d}, \mathrm{p})\right]=-1888.73772805$

$\begin{array}{lrrr}01 & & & \\ \mathrm{C} & 0.47226700 & 4.86149400 & -0.21382700 \\ \mathrm{H} & 1.52065900 & 5.11846500 & -0.03198100 \\ \mathrm{H} & 0.17285700 & 5.29117100 & -1.17538400 \\ \mathrm{H} & -0.13795200 & 5.31713900 & 0.57161900 \\ \mathrm{C} & 0.27464500 & 3.35811500 & -0.24499900 \\ \mathrm{H} & -0.77206800 & 3.08958700 & -0.42264500 \\ \mathrm{H} & 0.88211100 & 2.89200800 & -1.03209600 \\ \mathrm{O} & 0.67804900 & 2.83982100 & 1.04691100 \\ \mathrm{C} & 0.47149600 & 1.45786900 & 1.23553100 \\ \mathrm{H} & -0.41059600 & 1.09210800 & 0.69607800 \\ \mathrm{C} & 0.36497800 & 1.15471400 & 2.73068200 \\ \mathrm{H} & 0.27246500 & 0.07912600 & 2.88742600 \\ \mathrm{H} & -0.53077700 & 1.65673800 & 3.10293800\end{array}$




$\begin{array}{lrrr}\mathrm{S} & -3.46965400 & 0.53135200 & -0.30122600 \\ \mathrm{O} & -4.48970100 & 1.50021000 & 0.08848100 \\ \mathrm{O} & -3.16297500 & -0.37883800 & 0.96916000 \\ \mathrm{C} & -4.15173500 & -0.63331800 & -1.49019000 \\ \mathrm{H} & -3.42093700 & -1.42914900 & -1.64421100 \\ \mathrm{H} & -4.33721000 & -0.08083200 & -2.41266600 \\ \mathrm{H} & -5.08186700 & -1.02558200 & -1.07767500 \\ \mathrm{O} & -2.19447900 & 1.04372300 & -0.85392000 \\ \mathrm{H} & 1.23621300 & 1.54577500 & 3.26318900 \\ \mathrm{~S} & -0.22902800 & -2.53874700 & 0.26763700 \\ \mathrm{O} & 0.37603400 & -2.45932200 & -1.09672200 \\ \mathrm{O} & -1.72827400 & -2.33465200 & 0.24149500 \\ \mathrm{C} & -0.02253700 & -4.24330100 & 0.81409900 \\ \mathrm{H} & -0.53127600 & -4.89110300 & 0.09928400 \\ \mathrm{H} & -0.46529100 & -4.33709300 & 1.80646600 \\ \mathrm{H} & 1.04542800 & -4.46360600 & 0.84362300 \\ \mathrm{O} & 0.45164800 & -1.67010700 & 1.27249800 \\ \mathrm{H} & -2.50698300 & -1.14029100 & 0.73370600 \\ \mathrm{C} & 1.64676100 & 0.75206500 & 0.63814700 \\ \mathrm{~N} & 2.65562600 & 0.39551500 & 0.21301400 \\ \mathrm{C} & 3.82853700 & -0.14492300 & -0.46957400 \\ \mathrm{C} & 3.69776500 & -1.67789100 & -0.45378900 \\ \mathrm{H} & 2.77596300 & -2.00105800 & -0.94305500 \\ \mathrm{H} & 3.69778800 & -2.05614200 & 0.57205300 \\ \mathrm{H} & 4.55767500 & -2.09858200 & -0.98344400 \\ \mathrm{C} & 5.06816300 & 0.32987400 & 0.30721200 \\ \mathrm{H} & 5.05967600 & -0.04827500 & 1.33339000 \\ \mathrm{H} & 5.96159800 & -0.05279100 & -0.19382300 \\ \mathrm{H} & 5.12708200 & 1.42176300 & 0.33103300 \\ \mathrm{C} & 3.80120800 & 0.41682500 & -1.90331200 \\ \mathrm{H} & 4.63117200 & -0.02471000 & -2.46244400 \\ \mathrm{H} & 2.85815100 & 0.17609400 & -2.39867300 \\ \mathrm{H} & 3.92600800 & 1.50365700 & -1.89839700 \\ \mathrm{O} & 0.38566600 & 0.23750100 & -1.98409800 \\ \mathrm{H} & 0.35728700 & -0.72621200 & -1.81474700 \\ \mathrm{H} & -0.53469000 & 0.52568800 & -1.87354500\end{array}$




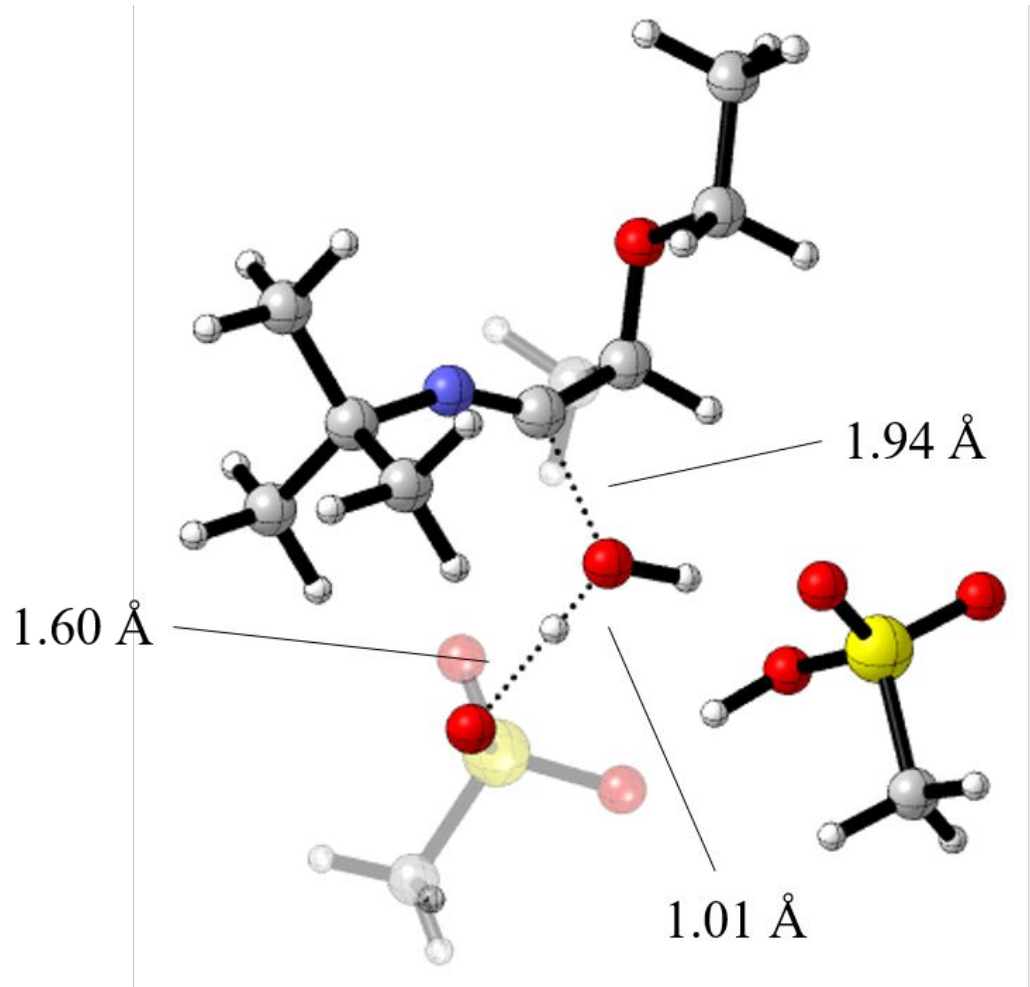

Figure S26. Optimized Structure of $\mathbf{T S 3}_{\mathrm{H}}$.

Zero-point correction $=$

Thermal correction to Energy=

Thermal correction to Enthalpy=

Thermal correction to Gibbs Free Energy=

Sum of electronic and zero-point Energies $=$

Sum of electronic and thermal Energies=

Sum of electronic and thermal Enthalpies=

Sum of electronic and thermal Free Energies=
0.399055 (Hartree/Particle)

0.428521

0.429465

0.334561

$-1887.919480$

$-1887.890015$

$-1887.889070$

$-1887.983974$

Number of Imaginary Frequencies $=1$

E (Single Point Energy) $\left[\operatorname{IEFPCM}_{(\mathrm{THF})} \mathrm{B} 3 \mathrm{LYP} / 6-311++\mathrm{G}(2 \mathrm{~d}, \mathrm{p})\right]=-1888.72836949$

01

$\begin{array}{lrrr}\mathrm{C} & 3.53460100 & 4.27527300 & 0.44715400 \\ \mathrm{H} & 4.46121900 & 3.78577700 & 0.76165000 \\ \mathrm{H} & 3.76321100 & 4.95179500 & -0.38285700 \\ \mathrm{H} & 3.15589400 & 4.86923700 & 1.28405400 \\ \mathrm{C} & 2.50452300 & 3.24954100 & 0.01011300 \\ \mathrm{H} & 1.57191600 & 3.73550700 & -0.30987900 \\ \mathrm{H} & 2.88321900 & 2.65479700 & -0.83537800 \\ \mathrm{O} & 2.23124000 & 2.38779300 & 1.12449500 \\ \mathrm{C} & 1.21730400 & 1.43489100 & 0.89841000\end{array}$




$\begin{array}{lrrr}\mathrm{H} & 0.38674400 & 1.86924300 & 0.33323100 \\ \mathrm{C} & 0.72782900 & 0.90114900 & 2.24643200 \\ \mathrm{H} & 0.01904300 & 0.07811700 & 2.12122000 \\ \mathrm{H} & 0.23949700 & 1.71976000 & 2.78036000 \\ \mathrm{~S} & -3.00095800 & 1.83210400 & -0.47682800 \\ \mathrm{O} & -3.45523200 & 3.17103800 & -0.12047300 \\ \mathrm{O} & -3.20986900 & 0.89303600 & 0.78264900 \\ \mathrm{C} & -4.08877200 & 1.12152600 & -1.71979200 \\ \mathrm{H} & -3.80524400 & 0.07751200 & -1.86379100 \\ \mathrm{H} & -3.95302600 & 1.69911000 & -2.63543900 \\ \mathrm{H} & -5.11256100 & 1.20552700 & -1.35368800 \\ \mathrm{O} & -1.60951500 & 1.69683900 & -0.98203100 \\ \mathrm{H} & 1.58074500 & 0.55055400 & 2.83388800 \\ \mathrm{~S} & -1.62561000 & -2.40040400 & 0.52130300 \\ \mathrm{O} & -0.58208300 & -2.48069200 & -0.56799200 \\ \mathrm{O} & -2.76901700 & -1.49746200 & 0.09768000 \\ \mathrm{C} & -2.34782500 & -4.04532000 & 0.60698900 \\ \mathrm{H} & -2.76290300 & -4.29073000 & -0.37097400 \\ \mathrm{H} & -3.12772700 & -4.02626000 & 1.36921100 \\ \mathrm{H} & -1.55633200 & -4.74386200 & 0.88139800 \\ \mathrm{O} & -1.07430300 & -2.06307000 & 1.85037900 \\ \mathrm{H} & -2.97089700 & -0.09590000 & 0.57714700 \\ \mathrm{C} & 1.79217900 & 0.28595100 & 0.08915500 \\ \mathrm{~N} & 2.74762300 & -0.40315100 & -0.01982600 \\ \mathrm{C} & 3.45813100 & -1.51656300 & -0.65267300 \\ \mathrm{C} & 2.90515500 & -2.81384300 & -0.03093100 \\ \mathrm{H} & 1.83518500 & -2.92339500 & -0.22532400 \\ \mathrm{H} & 3.06820900 & -2.82543200 & 1.05068200 \\ \mathrm{H} & 3.43550700 & -3.66512000 & -0.46900000 \\ \mathrm{C} & 4.95225800 & -1.34591800 & -0.33041100 \\ \mathrm{H} & 5.11982700 & -1.34475400 & 0.75027600 \\ \mathrm{H} & 5.51642300 & -2.17402400 & -0.76920700 \\ \mathrm{H} & 5.33865600 & -0.40900600 & -0.74290600 \\ \mathrm{C} & 3.21439100 & -1.47321300 & -2.17182400 \\ \mathrm{H} & 3.76330100 & -2.29597400 & -2.64006800 \\ \mathrm{H} & 2.15414700 & -1.57646400 & -2.40801800 \\ \mathrm{H} & 3.57638400 & -0.53277100 & -2.59828800 \\ & 0.38785000 & -0.13806800 & -1.17726900 \\ \mathrm{H} & -0.37908900 & -1.05722800 & -0.97283600 \\ \mathrm{H} & & 0.48268300 & -1.15353700\end{array}$




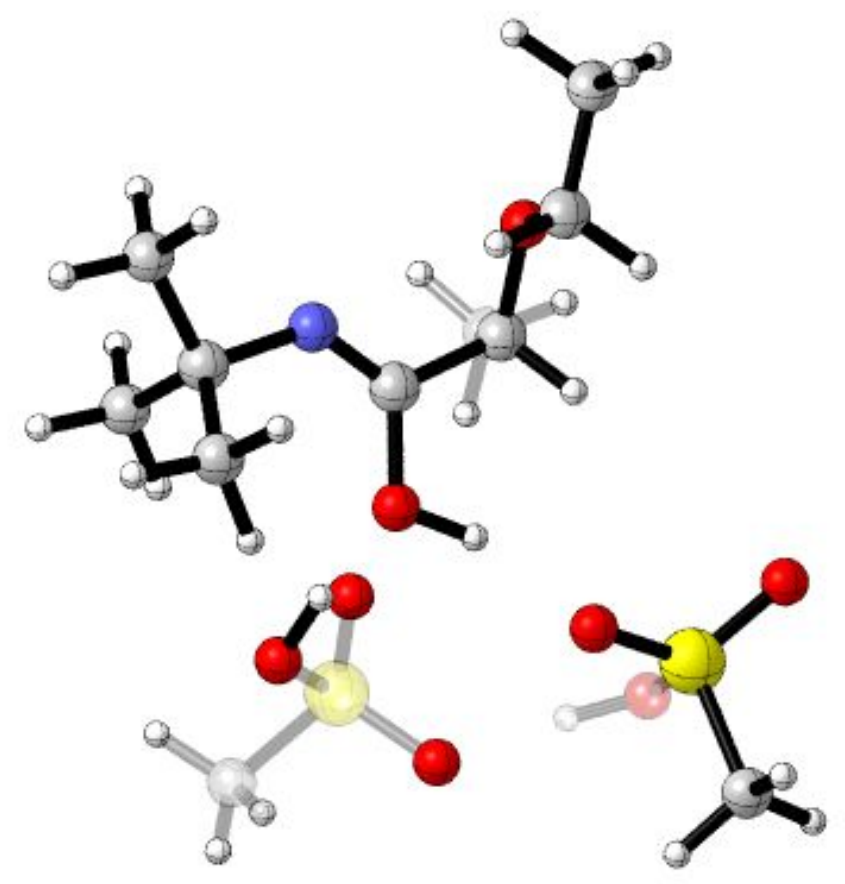

Figure S27. Optimized Structure of IM7.

Zero-point correction=

Thermal correction to Energy=

Thermal correction to Enthalpy=

Thermal correction to Gibbs Free Energy=

Sum of electronic and zero-point Energies $=$

Sum of electronic and thermal Energies=

Sum of electronic and thermal Enthalpies=

Sum of electronic and thermal Free Energies=
0.400790 (Hartree/Particle)

0.430429

0.431373

0.334954

$-1887.936168$

$-1887.906529$

$-1887.905585$

$-1888.002003$

Number of Imaginary Frequencies $=0$

E (Single Point Energy) $\left[\operatorname{IEFPCM}_{(\mathrm{THF})} \mathrm{B} 3 \mathrm{LYP} / 6-311++\mathrm{G}(2 \mathrm{~d}, \mathrm{p})\right]=-1888.74541873$

01

$\begin{array}{lrrr}\mathrm{C} & 4.46733200 & 3.40196100 & 0.60922800 \\ \mathrm{H} & 5.22753500 & 2.69965500 & 0.96415600 \\ \mathrm{H} & 4.88880600 & 3.98227400 & -0.21881100 \\ \mathrm{H} & 4.22314200 & 4.08833500 & 1.42595400 \\ \mathrm{C} & 3.22851400 & 2.65331300 & 0.14705400 \\ \mathrm{H} & 2.46122300 & 3.35682700 & -0.21598100 \\ \mathrm{H} & 3.47825000 & 1.96636000 & -0.67301600 \\ \mathrm{O} & 2.71227400 & 1.92009400 & 1.25643700 \\ \mathrm{C} & 1.51392900 & 1.22245300 & 1.00539000 \\ \mathrm{H} & 0.79109100 & 1.90073800 & 0.51949500 \\ \mathrm{C} & 0.95520000 & 0.77573500 & 2.35933900\end{array}$




$\begin{array}{lccc}\mathrm{H} & 0.03533800 & 0.19527800 & 2.24502000 \\ \mathrm{H} & 0.74370500 & 1.65515700 & 2.97332500 \\ \mathrm{~S} & -2.53785800 & 2.26898900 & -0.55080200 \\ \mathrm{O} & -2.20683500 & 3.52042800 & 0.11515900 \\ \mathrm{O} & -3.38876200 & 1.38214500 & 0.47776100 \\ \mathrm{C} & -3.71250200 & 2.58851300 & -1.87041600 \\ \mathrm{H} & -4.00849500 & 1.63565900 & -2.31067500 \\ \mathrm{H} & -3.20636300 & 3.21600500 & -2.60579000 \\ \mathrm{H} & -4.56701300 & 3.10972000 & -1.43773300 \\ \mathrm{O} & -1.45439900 & 1.43719400 & -1.11946200 \\ \mathrm{H} & 1.69994900 & 0.15848600 & 2.86963300 \\ \mathrm{~S} & -2.26948400 & -2.19617900 & 0.58630200 \\ \mathrm{O} & -1.07500600 & -2.42898700 & -0.45218800 \\ \mathrm{O} & -3.23821100 & -1.22643200 & 0.01372700 \\ \mathrm{C} & -3.01762800 & -3.81946200 & 0.50439500 \\ \mathrm{H} & -3.30718900 & -4.01424300 & -0.52824900 \\ \mathrm{H} & -3.89085000 & -3.79068000 & 1.15769600 \\ \mathrm{H} & -2.28747700 & -4.54669300 & 0.85983000 \\ \mathrm{O} & -1.75545700 & -1.91945900 & 1.92613600 \\ \mathrm{H} & -3.28880500 & 0.39900400 & 0.30469000 \\ \mathrm{C} & 1.70446900 & 0.00737200 & 0.08459100 \\ \mathrm{~N} & 2.79442500 & -0.60956500 & -0.00283900 \\ \mathrm{C} & 3.10821300 & -1.79284000 & -0.82510500 \\ \mathrm{C} & 2.52255900 & -3.05005400 & -0.15014900 \\ \mathrm{H} & 1.42976600 & -3.04519900 & -0.15453400 \\ \mathrm{H} & 2.86209200 & -3.11686100 & 0.88834100 \\ \mathrm{H} & 2.85761500 & -3.94937700 & -0.67894500 \\ \mathrm{C} & 4.64663600 & -1.89792800 & -0.82614200 \\ \mathrm{H} & 5.02252500 & -1.96660200 & 0.19899600 \\ \mathrm{H} & 4.97714100 & -2.78071900 & -1.38459300 \\ \mathrm{H} & 5.09076400 & -1.00974200 & -1.28724900 \\ \mathrm{C} & 2.61756100 & -1.67093600 & -2.28367000 \\ \mathrm{H} & 3.00617700 & -2.50927300 & -2.87238200 \\ \mathrm{H} & 1.52906800 & -1.67414600 & -2.35856300 \\ \mathrm{H} & 2.98831600 & -0.74419200 & -2.73513400 \\ \mathrm{O} & 0.52064700 & -0.35662100 & -0.57950200 \\ \mathrm{H} & -0.42025300 & -1.65366900 & -0.45797500 \\ \mathrm{H} & -0.09925900 & 0.40423400 & -0.66725000 \\ & & & \\ & & & \end{array}$




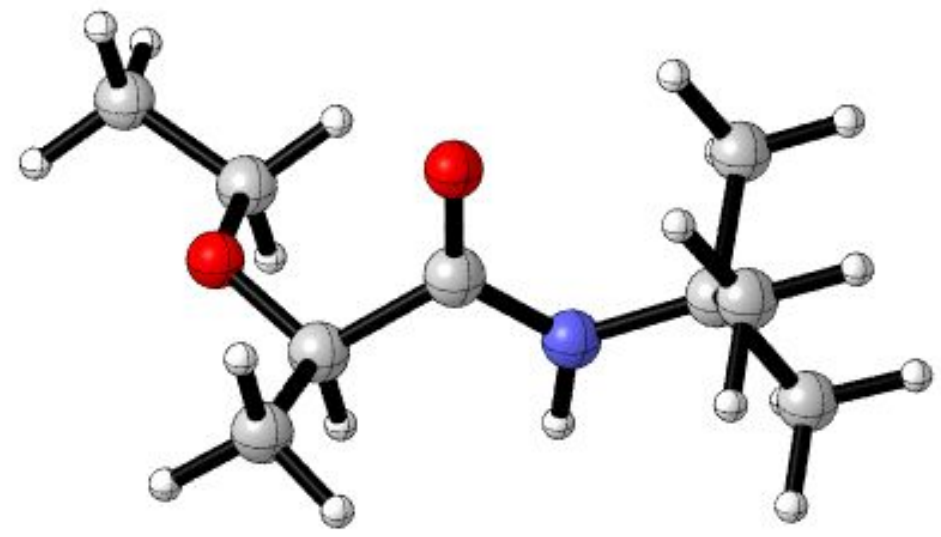

Figure S28. Optimized Structure of 3'.

Zero-point correction=

Thermal correction to Energy=

Thermal correction to Enthalpy=

Thermal correction to Gibbs Free Energy=

Sum of electronic and zero-point Energies $=$

Sum of electronic and thermal Energies=

Sum of electronic and thermal Enthalpies=

Sum of electronic and thermal Free Energies=
0.275463 (Hartree/Particle)

0.290752

0.291697

0.232907

$-559.389058$

$-559.373768$

$-559.372824$

$-559.431614$

Number of Imaginary Frequencies $=0$

E (Single Point Energy) $\left[\operatorname{IEFPCM}_{(\mathrm{THF})} \mathrm{B} 3 \mathrm{LYP} / 6-311++\mathrm{G}(2 \mathrm{~d}, \mathrm{p})\right]=-559.80513997$

01

$\begin{array}{lrrr}\mathrm{C} & -3.98645500 & -1.50404000 & -0.04935000 \\ \mathrm{H} & -4.11342000 & -1.37937000 & -1.12873300 \\ \mathrm{H} & -4.10880700 & -2.56476500 & 0.19486800 \\ \mathrm{H} & -4.77231800 & -0.93687700 & 0.45911500 \\ \mathrm{C} & -2.61082900 & -1.02273800 & 0.37982900 \\ \mathrm{H} & -2.47609300 & -1.14915600 & 1.46763900 \\ \mathrm{H} & -1.82632400 & -1.59935200 & -0.13005600 \\ \mathrm{O} & -2.49676200 & 0.35871600 & 0.04350700 \\ \mathrm{C} & -1.29154600 & 0.97392300 & 0.44597600 \\ \mathrm{H} & -1.16963800 & 0.85555900 & 1.53899000 \\ \mathrm{C} & -1.40414700 & 2.45695200 & 0.09673600 \\ \mathrm{H} & -0.51403100 & 3.00481200 & 0.42249000 \\ \mathrm{H} & -2.28222600 & 2.88510700 & 0.58823400 \\ \mathrm{H} & -1.51318300 & 2.56932200 & -0.98514900 \\ \mathrm{C} & -0.06085800 & 0.35914800 & -0.27050700 \\ \mathrm{~N} & 1.02409500 & 0.17000800 & 0.53482300\end{array}$




$\begin{array}{lrrr}\mathrm{C} & 2.36580300 & -0.30311900 & 0.10881600 \\ \mathrm{C} & 3.24144600 & -0.31415200 & 1.37205300 \\ \mathrm{H} & 2.83367000 & -0.99155700 & 2.13183900 \\ \mathrm{H} & 3.32608500 & 0.68948300 & 1.80542900 \\ \mathrm{H} & 4.25012800 & -0.65778100 & 1.12565600 \\ \mathrm{C} & 2.95819800 & 0.66459200 & -0.93300300 \\ \mathrm{H} & 3.04699500 & 1.67339900 & -0.51554600 \\ \mathrm{H} & 3.95736800 & 0.32767800 & -1.22966300 \\ \mathrm{H} & 2.32655700 & 0.70810900 & -1.82250900 \\ \mathrm{C} & 2.26083700 & -1.72699700 & -0.47030500 \\ \mathrm{H} & 3.25409800 & -2.08649400 & -0.76064600 \\ \mathrm{H} & 1.85145900 & -2.41700200 & 0.27550200 \\ \mathrm{H} & 1.61406400 & -1.73792100 & -1.34976600 \\ \mathrm{O} & -0.07772900 & 0.10810600 & -1.47215800 \\ \mathrm{H} & 0.90818700 & 0.38076700 & 1.51628400\end{array}$




\section{References}

${ }^{1}$ Burés, J. Variable Time Normalization Analysis: General Graphical Elucidation of Reaction Orders from Concentration Profiles. Angew. Chem. Int. Ed. 2016, 55, 16084-16087. 